\title{
Rhodium-Catalyzed Asymmetric Allylic Substitution with Boronic Acid Nucleophiles
}

\author{
Frederic Menard, Timothy M. Chapman, Chris Dockendorff and Mark Lautens*
}

\section{Supporting Information}

\section{Table of Contents}

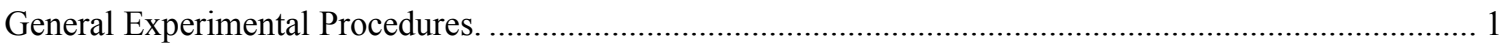

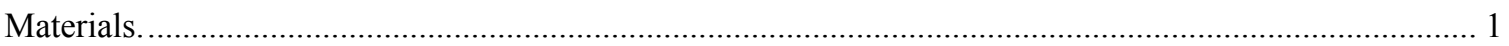

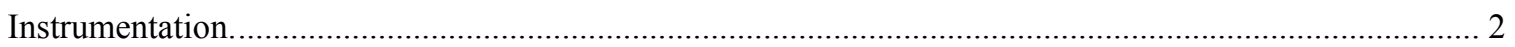

Determination of the Enantiomeric Excesses by HPLC Analysis. ....................................................... 2

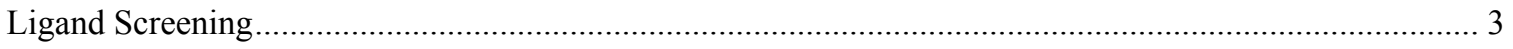

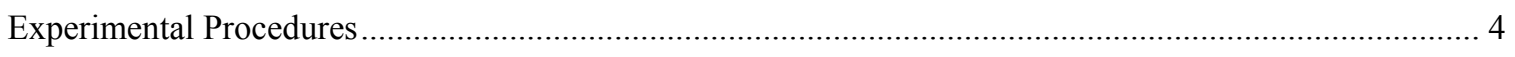

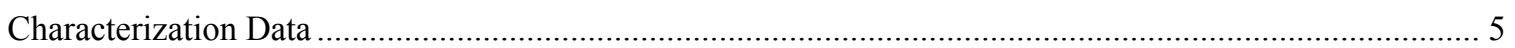

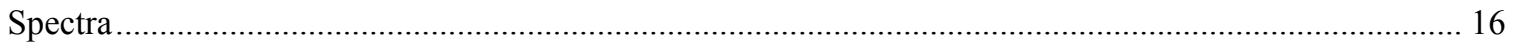

General Experimental Procedures. All reactions were carried out under argon atmosphere, in flamedried, single-neck, roundbottom flasks fitted with rubber septa, with magnetic stirring unless otherwise noted. Air- or water-sensitive liquids and solutions were transferred via syringe or stainless steel cannula. Where necessary (so noted), solutions were deoxygenated by successive freeze-pump-thaw cycles $(\geq$ three iterations). Organic solutions were concentrated by rotary evaporation at $23-40{ }^{\circ} \mathrm{C}$ at 40 Torr (house vacuum). Analytical thin layer chromatography (TLC) was performed with Silicycle ${ }^{\mathrm{TM}}$ normal phase glass plates (0.25 mm, 60-A pore size, 230-400 mesh). Visualization was accomplished with $254 \mathrm{~nm}$ UV light and/or by immersion in potassium permanganate $\left(\mathrm{KMnO}_{4}\right)$, or acidic aqueous-ethanolic vanillin solution, followed by brief heating using a heat gun. Purification of reaction products was generally done by flash chromatography with Silicycle ${ }^{\text {TM }}$ Ultra-Pure 230-400 mesh silica gel, as described by Still et al. ${ }^{1}$

Materials. $[\mathrm{Rh}(\mathrm{cod}) \mathrm{Cl}]_{2}$ was purchased from Strem Chemicals, Inc. and used as received. $[\mathrm{Rh}(\mathrm{cod}) \mathrm{OH}]_{2}$ was prepared from $[\mathrm{Rh}(\mathrm{cod}) \mathrm{Cl}]_{2}$ by a literature procedure. ${ }^{2}$ All chiral phosphine ligands were purchased from Strem Chemicals Inc. All boronic acids were obtained from Aldrich and used without further purification, unless otherwise noted. Tetrahydrofuran, 1,4-dioxane and toluene were purified by distillation under $\mathrm{N}_{2}$ from $\mathrm{Na}$ /benzophenone immediately prior to use. Ether and dichloromethane were purified by the

${ }^{1}$ Still, W. C.; Kahn, M.; Mitra, A. J. Org. Chem. 1978, 43, 2923.

${ }^{2}$ Uson, R.; Oro, L.A.; Cabeza, J. A. Inorg. Synth. 1985, 23, 126. 
method of Pangborn et al. ${ }^{3}$ Cis-2-cyclopentene-1,4-diol was prepared by a literature procedure and characterization data was fully consistent with that reported in the literature. ${ }^{4}$ Meso-cis-cyclopent-2-ene1,4-diethyl carbonate (1) was prepared following a literature procedure. ${ }^{5}$

Instrumentation. Proton nuclear magnetic resonance spectra $\left({ }^{1} \mathrm{H}\right.$ NMR) spectra and carbon nuclear magnetic resonance spectra $\left({ }^{13} \mathrm{C}\right.$ NMR) were recorded at $23{ }^{\circ} \mathrm{C}$ with a Varian $400(400 \mathrm{MHz} / 100 \mathrm{MHz})$ NMR spectrometer equipped with ATB8123-400 probe, or a Varian Mercury $400(400 \mathrm{MHz} / 100 \mathrm{MHz})$ NMR spectrometer equipped with a Nalorac $4 \mathrm{~N}-400$ probe. Recorded shifts for protons are reported in parts per million ( $\delta$ scale) downfield from tetramethylsilane and are referenced to residual protium in the NMR solvents $\left(\mathrm{CHCl}_{3}: \delta 7.26, \mathrm{CHDCl}_{2}: \delta 5.29, \mathrm{C}_{6} \mathrm{HD}_{5}: \delta 7.15, \mathrm{CD}_{2} \mathrm{HOD}: \delta 3.30\right)$. Chemical shifts for carbon resonances are reported in parts per million ( $\delta$ scale) downfield from tetramethylsilane and are referenced to the carbon resonances of the solvent $\left(\mathrm{CDCl}_{3}: \delta 77.0, \mathrm{CH}_{2} \mathrm{Cl}_{2}: \delta 53.8, \mathrm{C}_{6} \mathrm{D}_{6}: \delta 128.0, \mathrm{CD}_{3} \mathrm{OD}\right.$ : $\delta$ 49.2). Data are represented as follows: chemical shift, multiplicity $(\mathrm{s}=$ singlet, $\mathrm{d}=$ doublet, $\mathrm{t}=$ triplet, $\mathrm{q}=$ quartet, qn = quintuplet, $\mathrm{sx}=$ sextet, $\mathrm{sp}=$ septuplet, $\mathrm{m}=$ multiplet, $\mathrm{br}=$ broad), integration and coupling constant $(\mathrm{J}, \mathrm{Hz})$. Infrared (IR) spectra were obtained using a Perkin-Elmer Spectrum 1000 FT-IR spectrometer as a neat film on a $\mathrm{NaCl}$ plate. Data is presented as follows: frequency of absorption $\left(\mathrm{cm}^{-1}\right)$, intensity of absorption $(\mathrm{s}=$ strong, $\mathrm{m}=$ medium, $\mathrm{w}=$ weak, $\mathrm{br}=$ broad), and assignment. High resolution mass spectra were obtained from a SI2 Micromass 70S-250 mass spectrometer (EI) or an ABI/Sciex Qstar mass spectrometer (ESI). Melting points were taken on a Fisher-Johns melting point apparatus and are uncorrected. Optical rotations were measured in a 5.0 or $10.0 \mathrm{~cm}$ cell with a Rudolph Autopol IV polarimeter digital polarimeter equipped with a sodium lamp source $(589 \mathrm{~nm})$, and are reported as follows: $[\alpha]_{\mathrm{D}}{ }^{\mathrm{T}^{\circ} \mathrm{C}}(\mathrm{c}=\mathrm{g} / 100 \mathrm{~mL}$, solvent). Reported readings are an average of at least two measurements for each sample.

Determination of the Enantiomeric Excesses by HPLC Analysis. The enantiomeric excess (ee) of substituted products was determined after hydrolysis of the carbonate to the parent alcohols, followed by HPLC analysis of the crude reaction mixture (see following section for details). Unless otherwise noted, enantiomeric excesses of the deprotected products were determined using a Daicel Chemical Industries Ltd, Chiralcel OD-H column (fitted with a $5.0 \mathrm{~cm}$ Chiralcel OD-H guard column), flow rate $1.0 \mathrm{~mL} / \mathrm{min}, 30^{\circ} \mathrm{C}$, typically using a 1:99 i-PrOH:hexane solvent mixture., injection $4.0 \mathrm{uL}$.

\footnotetext{
${ }^{3}$ Pangborn, A. B.; Giardello, M. A.; Grubbs, R. H.; Rosen, R. K.; Timmers, F. J. Organometallics 1996, 15, 1518.

${ }^{4}$ Kaneko, C.; Sugimoto, A.; Tanaka, S. Synthesis 1974, 876.

${ }^{5}$ Arnau, N.; Cortés, J.; Moreno-Mañas, M.; Pleixats, R.; Villaroya, M. J. Heterocyclic Chem. 1997, 34, 233. 


\section{Ligand Screening}

The biaryl bisphosphine ligands were the only class of ligands which showed any appreciable activity. The use of Tol-BINAP (entry 2) led to an increase in the enantioselectivity of the 1,2-adduct, but a decrease in the enantioselectivity of the 1,4-adduct. Cl-OMe-BIPHEP (entry 6) gave excellent enantioselectivities for both adducts, however no regioselectivity was observed. The most useful result was obtained with Xyl-PPhos (entry 10), which gave excellent enantioselectivity and good regioselectivity for the synthesis of the 1,2-adduct 2 .
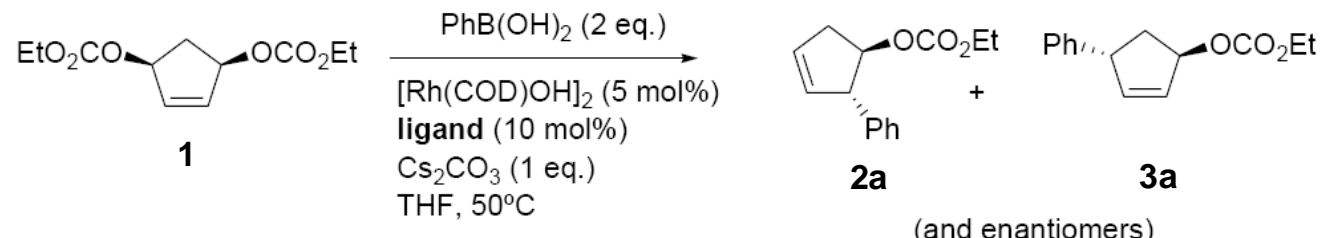

$2 \mathbf{a}$

$3 a$

(and enantiomers)

\begin{tabular}{|c|c|c|c|c|c|}
\hline Entry & Ligand $^{b}$ & Conv. $\%^{c}$ & $2: 3^{C}$ & $2 \mathrm{ee} \% \%^{d}$ & 3 еe $\%{ }^{d}$ \\
\hline 1 & BINAP & 45 & $2: 1$ & 76 & 72 \\
\hline 2 & Tol-BINAP & 32 & $1: 1$ & 95 & 32 \\
\hline 3 & Xyl-BINAP & 59 & $2: 1$ & 82 & 60 \\
\hline 4 & ChiraPhos & 2 & -- & -- & -- \\
\hline 5 & MonoPhos & 3 & -- & -- & -- \\
\hline 6 & Cl-MeO-BIPHEP & 60 & $1: 1$ & 90 & $>98$ \\
\hline 7 & Me-DuPhos & 0 & -- & -- & -- \\
\hline 8 & $i$-Pr-POX & 0 & -- & -- & -- \\
\hline 9 & SolPhos & 11 & -- & -- & -- \\
\hline 10 & Xyl-P-Phos & 65 & $13: 1$ & 88 & $>98$ \\
\hline 11 & $\mathrm{C}_{2}$-Ferriphos & 3 & -- & -- & -- \\
\hline 12 & KenPhos & 1 & -- & -- & -- \\
\hline 13 & $\mathrm{PPF}-\mathrm{P}(t-\mathrm{Bu})_{2}$ & 0 & -- & -- & -- \\
\hline 14 & QUINAP & 3 & -- & -- & -- \\
\hline 15 & Trost & 0 & -- & -- & -- \\
\hline 16 & BDPP & 0 & -- & -- & -- \\
\hline
\end{tabular}

${ }^{a}$ Unless otherwise noted, substrate, $\mathrm{PhB}(\mathrm{OH})_{2}$, catalyst, ligand, and base were added to a flask with stir bar and sealed and flushed with nitrogen before THF was added. ${ }^{b}$ Determined by $1 \mathrm{H}$ NMR.

${ }^{d}$ Determined by chiral HPLC on the deprotected products.

Absolute stereochemistries of the products were assigned by MTPA ester derivatives ${ }^{6}$ of $4 \mathbf{a}$, and by correlation of optical rotations with reported data of known compounds 2 and 3 . $^{7}$

\footnotetext{
${ }^{6}$ See reference 12 .

${ }^{7}$ a) Piarulli, U.; Daubos, P.; Claverie, C.; Roux, M.; Gennari, C. Angew. Chem., Int. Ed. 2003, 42, 234. b) Kobayashi, Y.; Murugesh, M. G.; Nakano, M.; Takahisa, E.; Usmani, S. B.; Ainai, T. J. Org. Chem. 2002, 67, 7110. c) Tueting, D. R.; Echavarren, A. M.; Stille, J. K. Tetrahedron 1989, 45, 979. d) Marino, J. P.; Fernández de la Pradilla, R.; Laborde, E. J. Org. Chem. 1987, 52, 4898.
} 


\section{Experimental Procedures}

Typical procedure: Desymmetrization by Rhodium-catalyzed Asymmetric Allylic Substitution with Phenylboronic acid on cis-cyclopent-2-ene-1,4-diethyl carbonate (1).

To a 1 dram vial equipped with a magnetic stir bar was added rhodium cyclooctadiene hydroxide, $[\mathrm{Rh}(\mathrm{cod}) \mathrm{OH}]_{2}(3.7 \mathrm{mg}, 0.0082 \mathrm{mmol}),(S)-X y l-P-P H O S(14.9 \mathrm{mg}, 0.020 \mathrm{mmol})$ and $\mathrm{Cs}_{2} \mathrm{CO}_{3}(53 \mathrm{mg}, 0.164$ mmol). The vial was sealed and flushed with argon, then distilled THF $(0.8 \mathrm{~mL})$ was added and the mixture was stirred for $30 \mathrm{~min}$ on a $60{ }^{\circ} \mathrm{C}$ oil bath. Cis-2-cyclopentene-1,4-diethyl carbonate 1 (40 mg, $0.164 \mathrm{mmol})$ and phenylboronic acid $(40 \mathrm{mg}, 0.32 \mathrm{mmol})$ were added together as a solution in distilled THF $(0.8 \mathrm{~mL})$ and the reaction mixture was heated on a $60^{\circ} \mathrm{C}$ oil bath. After $1 \mathrm{~h}$, THF $(5 \mathrm{~mL})$ and hexanes $(5 \mathrm{~mL})$ were added to the reaction mixture and it was concentrated with silica gel, then applied to the top of a column of silica gel and purified by column chromatography (5 to $20 \%$ EtOAc/hexane as elution gradient). An inseparable mixture of the mono-carbonates $\mathbf{2}$ and $\mathbf{3}$ was recovered as a colourless oil, $33 \mathrm{mg}$ (87\%). ${ }^{1} \mathrm{H}$ NMR analysis revealed an isomer ratio of $92: 8$ for the 1,2-product 2 over the 1,4-product 3 . The enantiomeric excess was determined on the deprotected alcohols (vide infra).

\section{Typical deprotection of carbonates}

The combined 1,2- and 1,4-mono-carbonates 2 and 3 isomers were dissolved in $\mathrm{MeOH}(0.02 \mathrm{M})$ and $\mathrm{K}_{2} \mathrm{CO}_{3}$ (10-14 equiv) was added. The white suspension was stirred at $\mathrm{rt}$ until complete conversion (usually $<3 \mathrm{~h}$ ). ${ }^{8}$ One volume of $\mathrm{NH}_{4} \mathrm{Cl}$ saturated aqueous solution was added and extracted three times with $\mathrm{CH}_{2} \mathrm{Cl}_{2}$. The combined organic layers were dried over $\mathrm{MgSO}_{4}$, filtered and concentrated under reduced pressure to yield a mixture of the alcohols $\mathbf{4}$ and $\mathbf{5}$. The crude products wre analyzed by HPLC (Daicel Chiralcel OD-H, 1:99 to 3:97 i-PrOH/hexane), and peaks due to the four enantiomers could be resolved. The 1,2- and 1,4-regioisomers 4 and $\mathbf{5}$ were separated and purified by column chromatography on silica gel (25\% EtOAc/hexane) when significant amounts of 5 were present. ${ }^{9}$

Note: The use (S)-biarylphosphines ligands (BINAP and P-PHOS analogs) all lead to the (-) enantiomers, which have the absolute stereochemistry represented in this paper. The use of the (R)-biarylphosphines yield the $(+)$ enantiomers, having the opposite stereochemistry of that represented. We arbitrarily opted to represent the $(1 R, 2 S)$-stereochemistry for consistency and to avoid unnecessary complications for the readers.

\footnotetext{
${ }^{8}$ It was verified that longer reaction times did not affect the ee (up to $48 \mathrm{~h}$ at $\mathrm{rt}$ ).

${ }^{9}$ When relevant, the regioisomers 5 were analyzed independently by HPLC, thus confirming retention times assignations. 


\section{Characterization Data}

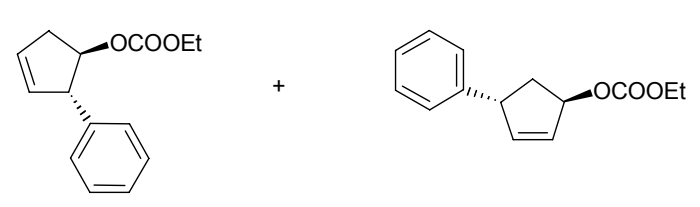

(2a) and (3a) : (1R, 2S)-trans-2-phenylcyclopent-3enyl-ethylcarbonate and $(1 R, 4 R)$-trans-4phenylcyclopent-2-enyl-ethylcarbonate. Prepared according to the general procedure described above (using (S)-Xylyl-P-PHOS). The crude product was purified by column chromatography (dry loaded). The inseparable regioisomers 2a and 3a were obtained as a colourless oil in $87 \%$ yield $\left(33.0 \mathrm{mg}\right.$ ), showing a $18: 1$ ratio of $2: 3$ by ${ }^{1} \mathrm{H}$ NMR spectroscopy. The regioisomers were submitted directly to the deprotection conditions and characterized as the free alcohols. Major regioisomer 2a: ${ }^{1} \mathbf{H}$ NMR $\left(400 \mathrm{MHz}, \mathrm{CDCl}_{3}\right): \delta$ 7.34-7.21 $(5 \mathrm{H}, \mathrm{m}), 5.94(1 \mathrm{H}, \mathrm{ddd}, J=5.9,4.1,2.0$ $\mathrm{Hz}), 5.82(1 \mathrm{H}, \mathrm{ddd}, J=5.8,4.2,2.0 \mathrm{~Hz}), 5.04(1 \mathrm{H}, \mathrm{dt}, J=6.6,2.6 \mathrm{~Hz}), 4.20(2 \mathrm{H}, \mathrm{q}, J=7.1 \mathrm{~Hz}), 4.00(1 \mathrm{H}$, br. s), 2.92 (1H, dddd, $J=17.8,6.3,4.0,2.3 \mathrm{~Hz}), 2.48(1 \mathrm{H}, \mathrm{dddd}, J=17.8,7.9,5.1,2.4 \mathrm{~Hz}), 1.31(3 \mathrm{H}, \mathrm{t}, J$ $=7.1 \mathrm{~Hz}) .{ }^{13} \mathrm{C}$ NMR $\left(100 \mathrm{MHz}, \mathrm{CDCl}_{3}\right): \delta 155.1,141.3,132.0,129.6,128.8,127.7,127.1,85.2,64.1$, 57.8, 39.0, 14.5. IR ( $\mathrm{NaCl}$, neat film): 3061, 3030, 2983, 2850, 1743, 1451, 1374, 1261, 1170, 1105, 1012 $\mathrm{cm}^{-1}$. Minor regioisomer 3a: ${ }^{1} \mathbf{H}$ NMR $\left(400 \mathrm{MHz}, \mathrm{CDCl}_{3}\right): \delta 7.37-7.23(5 \mathrm{H}, \mathrm{m}), 6.12(1 \mathrm{H}, \mathrm{dd}, J=5.6,2.0$ $\mathrm{Hz}), 6.01(1 \mathrm{H}, \mathrm{dt}, J=5.5,2.3 \mathrm{~Hz}), 5.70(1 \mathrm{H}, \mathrm{ddd}, J=7.1,4.1,2.0 \mathrm{~Hz}), 4.13(2 \mathrm{H}, \mathrm{q}, J=7.1 \mathrm{~Hz}), 4.09-4.05$ $(1 \mathrm{H}, \mathrm{m}), 2.46-2.36(1 \mathrm{H}, \mathrm{m}), 2.06(1 \mathrm{H}, \mathrm{ddd}, J=13.0,7.1,5.9 \mathrm{~Hz}), 1.25(3 \mathrm{H}, \mathrm{t}, J=7.1 \mathrm{~Hz})$.

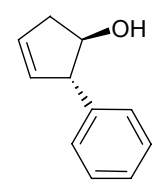

(4a) : (1R, 2S)-trans-2-phenylcyclopent-3-enol. Obtained by methanolysis of the isolated carbonates mixture (vide supra) and purified by column chromatography $(25 \%$ EtOAc/hexanes). The alcohol 4a was quantitavely isolated as a colourless oil. The characterization data was fully concordant with that already reported in the literature. ${ }^{10}{ }^{1} \mathbf{H}$ NMR (400 MHz, $\left.\mathrm{CDCl}_{3}\right): \delta 7.31-7.14(5 \mathrm{H}, \mathrm{m}), 5.87(1 \mathrm{H}, \mathrm{ddt}, J=6.2,4.4,2.2 \mathrm{~Hz}), 5.75(1 \mathrm{H}, \mathrm{ddd}, J=6.1$, 4.2, $2.1 \mathrm{~Hz}), 4.29-4.25(1 \mathrm{H}, \mathrm{m}), 3.74(1 \mathrm{H}$, ddd, $J=5.7,3.7,1.8 \mathrm{~Hz}), 2.78(1 \mathrm{H}$, dddd, $J=16.9,6.3,3.9,1.9$, $\mathrm{Hz}), 2.35$ (1H, dddd, $J=16.9,6.1,3.9,1.8 \mathrm{~Hz}), 1.84\left(1 \mathrm{H}\right.$, br. s, alcohol). ${ }^{13} \mathrm{C}$ NMR $\left(100 \mathrm{MHz}, \mathrm{CDCl}_{3}\right): \delta$ 142.6, 132.3, 129.5, 128.6, 127.3, 126.6., 80.9, 60.7, 41.3. IR ( $\mathrm{NaCl}$, neat film): 3328 (br.), 3056, 3026, 2924, 2845, 1650, 1557, 1537, 1492, 1453, $1052 \mathrm{~cm}^{-1}$. MS m/z (rel. intensity): 160 (M+ $\left.\mathbf{M}^{+}, 84\right), 117$ (50), 104 (100), 91 (70). HRMS (ESI) ${ }^{+}$: Calc'd for $\mathrm{C}_{11} \mathrm{H}_{12} \mathrm{O}\left[\mathrm{M}^{+}\right]$: 160.088815 ; found $=160.089202$. The ee was determined by HPLC analysis : 92\% ee (Chiralcel OD-H, hexane/iPrOH $=99 / 1,1.0 \mathrm{~mL} / \mathrm{min}) ; \mathrm{t}_{\mathrm{R}}=31.1$ $\min (1 S, 2 R)$ minor; $\mathrm{t}_{\mathrm{R}}=38.2 \min (1 R, 2 S)$ major. $[\alpha]^{26.7}{ }_{589}=-228\left(\mathrm{c} 1.60, \mathrm{CHCl}_{3}\right.$, for $92 \%$ ee). ${ }^{11,12}$

(5a) : (1R, 4R)-trans-4-phenylcyclopent-2-enol. Colourless oil. The characterization data was fully concordant with that already reported in the literature. ${ }^{10 \mathrm{~b}}{ }^{1} \mathbf{H}$ NMR (400 $\left.\mathrm{MHz}, \mathrm{CDCl}_{3}\right): \delta$ 7.25-7.05 (5H, m), 6.00-5.94 $(2 \mathrm{H}, \mathrm{m}), 5.00-4.96(1 \mathrm{H}, \mathrm{m}), 4.08(1 \mathrm{H}$, ddt, $J=5.9,3.5,1.9 \mathrm{~Hz}), 2.22(1 \mathrm{H}, \mathrm{ddd}, J=14.0,8.0,2.7 \mathrm{~Hz}), 2.03(1 \mathrm{H}, \mathrm{ddd}, J=14.0$, $6.9,5.5 \mathrm{~Hz}), 1.47$ (1H, br. s).

\footnotetext{
${ }^{10}$ a) Tueting, D. R.; Echavarren, A. M.; Stille, J. K. Tetrahedron 1989, 45, 979. b) Kobayashi, Y.; Murugesh, M. G.; Nakano, M.; Takahisa, E.; Usmani, S. B.; Ainai, T. J. Org. Chem. 2002, 67, 7110.

${ }^{11}$ The enantiomeric ligands $(R)$-Xylyl-P-PHOS or $(R)$-BINAP gave the corresponding $(+)$-trans-cyclopentenol.

${ }^{12}$ The absolute stereochemistry of $\mathbf{4 a}$ was assigned by the Mosher method on the MTPA ester derivative of 4a (2 equiv (S)-MTPA acid, 2 equiv DCC and 0.2 equiv DMAP were stirred in DCM at rt for 10h; quantitative yield). For details, see : Dale, J. A., Mosher, H. S. J. Am. Chem. Soc. 1973, 95, 512.
} 


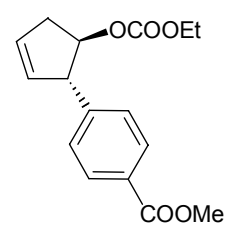

(2b) : (1R, 2S)-trans-2-(4-Methoxycarbonyl-phenyl)-cyclopent-3-enyl ethylcarbonate. Prepared according to the general procedure described above. The crude product was purified by column chromatography (dry loaded). The inseparable regioisomers 2 and 3 were obtained as a colorless oil in 95\% yield $(45 \mathrm{mg})$, showing a $>20: 1$ ratio of 2:3 by ${ }^{1} \mathrm{H}$ NMR spectroscopy. The regioisomers were submitted directly to the deprotection conditions and characterized as the free alcohols. Major regioisomer $\mathbf{2 b}$ : ${ }^{1} \mathbf{H} \mathbf{~ N M R}$ $\left(400 \mathrm{MHz}, \mathrm{CDCl}_{3}\right): \delta 7.97(2 \mathrm{H}, \mathrm{d}, J=8.3 \mathrm{~Hz}), 7.28(2 \mathrm{H}, \mathrm{d}, J=8.2 \mathrm{~Hz}), 5.98(1 \mathrm{H}, \mathrm{ddd}, J=5.9,4.2,2.2$ $\mathrm{Hz}), 5.80(1 \mathrm{H}, \mathrm{ddd}, J=5.9,4.0,2.0 \mathrm{~Hz}), 5.04(1 \mathrm{H}$, ddd, $J=6.3,2.5,2.5 \mathrm{~Hz}), 4.20(2 \mathrm{H}, \mathrm{q}, J=7.1 \mathrm{~Hz})$, $4.00(1 \mathrm{H}$, br. s), $2.92(1 \mathrm{H}, \mathrm{dddd}, J=17.8,6.3,4.0,2.3 \mathrm{~Hz}), 2.41(1 \mathrm{H}$, dddd, $J=17.8,7.9,5.1,2.3 \mathrm{~Hz})$, $1.31(3 \mathrm{H}, \mathrm{t}, J=7.1 \mathrm{~Hz}) .{ }^{13} \mathrm{C}$ NMR $\left(100 \mathrm{MHz}, \mathrm{CDCl}_{3}\right): \delta 166.9,154.9,146.4,131.1,130.1,129.9,128.9$, 127.6, 84.6, 64.0, 57.6, 52.0, 38.7, 14.2. IR (NaCl, neat film): 3061, 2982, 2952, 2925, 2853, 1741, 1722, $1651,1611,1436,1374,1262 \mathrm{~cm}^{-1}$.

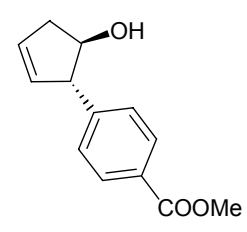

(4b) : (1R, 2S)-trans-2-(4-Methoxycarbonylphenyl)-cyclopent-3-enol. Obtained by methanolysis of the isolated carbonates mixture (vide supra) and purified by column chromatography (25\% EtOAc/hexanes). The alcohol $\mathbf{4 b}$ was quantitavely isolated as a colourless oil. ${ }^{1} \mathbf{H}$ NMR $\left(400 \mathrm{MHz}, \mathrm{CDCl}_{3}\right): \delta 7.97(2 \mathrm{H}, \mathrm{d}, J=8.2 \mathrm{~Hz}), 7.25(2 \mathrm{H}, \mathrm{d}, J$ $=8.3 \mathrm{~Hz}) 5.94(1 \mathrm{H}, \mathrm{ddd}, J=6.2,4.4,2.2 \mathrm{~Hz}), 5.76(1 \mathrm{H}, \mathrm{ddd}, J=6.3,4.4,2.2 \mathrm{~Hz}), 4.28$

$(1 \mathrm{H}, \mathrm{dt}, J=7.1,4.1 \mathrm{~Hz}), 3.90(3 \mathrm{H}, \mathrm{s}), 3.85-3.82(1 \mathrm{H}, \mathrm{m}), 2.81(1 \mathrm{H}, \mathrm{dddd}, J=17.1,6.8,3.7,2.2 \mathrm{~Hz}), 2.39$ $(1 \mathrm{H}$, dddd, $J=17.1,5.9,3.9,2.2 \mathrm{~Hz}), 1.95\left(1 \mathrm{H}\right.$, br. s, alcohol). ${ }^{13} \mathrm{C}$ NMR $\left(100 \mathrm{MHz}, \mathrm{CDCl}_{3}\right): \delta 167.0$, 148.0, 131.5, 130.2, 129.9, 128.6, 127.3, 80.8, 60.7, 52.0, 41.5. IR ( $\mathrm{NaCl}$, neat film): 3418 (br.), 3054, 2998, 2949, 2845, 1717, 1651, 1609, 1436, 1282, $1179 \mathrm{~cm}^{-1}$. MS m/z (rel. intensity): $218\left(\mathrm{M}^{+}, 100\right), 200$ (14), 187 (40), 162 (67), 131 (95), 115 (47). HRMS (ESI) ${ }^{+}$: Calc'd for $\mathrm{C}_{13} \mathrm{H}_{14} \mathrm{O}_{3}\left[\mathrm{M}^{+}\right]$: 218.0943; found = 218.0947. The ee was determined by HPLC analysis : $90 \%$ ee (Chiralcel OD-H, hexane/iPrOH $=96 / 4,1.0$ $\mathrm{mL} / \mathrm{min}) ; \mathrm{t}_{\mathrm{R}}=19.5 \min (1 S, 2 R)$ minor; $\mathrm{t}_{\mathrm{R}}=20.4 \min (1 R, 2 S)$ major. $[\alpha]^{24.8}=-354\left(\mathrm{c} 0.90, \mathrm{CHCl}_{3}\right.$, for $90 \%$ ee).

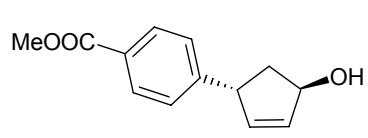

(5b) : (1R, 4R)-trans-4-(4-Methoxycarbonylphenyl)-cyclopent-2-enol. Colourless oil. ${ }^{1} \mathbf{H}$ NMR (400 MHz, $\left.\mathrm{CDCl}_{3}\right): \delta 7.97(2 \mathrm{H}, \mathrm{d}, J=8.2 \mathrm{~Hz}), 7.25$ $(2 \mathrm{H}, \mathrm{d}, J=8.3 \mathrm{~Hz}) 5.94(1 \mathrm{H}, \mathrm{ddd}, J=6.2,4.4,2.2 \mathrm{~Hz}), 5.76(1 \mathrm{H}, \mathrm{ddd}, J=6.3$, 4.4, $2.2 \mathrm{~Hz}), 4.28(1 \mathrm{H}, \mathrm{dt}, J=7.1,4.1 \mathrm{~Hz}), 3.90(3 \mathrm{H}, \mathrm{s}), 3.85-3.82(1 \mathrm{H}, \mathrm{m}), 2.81(1 \mathrm{H}$, dddd, $J=17.1,6.8$, 3.7, $2.2 \mathrm{~Hz}), 2.39$ (1H, dddd, $J=17.1,5.9,3.9,2.2 \mathrm{~Hz}), 1.95$ (1H, br. s, alcohol). ${ }^{13} \mathbf{C}$ NMR (100 MHz, $\left.\mathrm{CDCl}_{3}\right): \delta 167.0,148.0,131.5,130.2,129.9,128.6,127.3,80.8,60.7,52.0,41.5$.

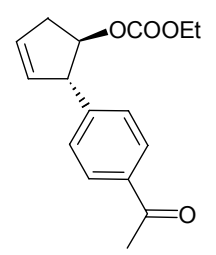

(2c) : (1R, 2S)-trans-2-(4-Acetylphenyl)-cyclopent-3-enyl ethylcarbonate. Prepared according to the general procedure described above. The crude product was purified by column chromatography (dry loaded). The inseparable regioisomers $\mathbf{2}$ and $\mathbf{3}$ were obtained as a colourless oil in $94 \%$ yield (42 mg), showing a $>20: 1$ ratio of 2:3 by ${ }^{1} \mathrm{H}$ NMR spectroscopy. The regioisomers were submitted directly to the deprotection conditions and characterized as the free alcohols. Major regioisomer 2c: ${ }^{1} \mathbf{H}$ NMR $(400 \mathrm{MHz}$, $\left.\mathrm{CDCl}_{3}\right): \delta 7.91(2 \mathrm{H}, \mathrm{d}, J=8.3 \mathrm{~Hz}), 7.32(2 \mathrm{H}, \mathrm{d}, J=8.3 \mathrm{~Hz}), 5.98(1 \mathrm{H}, \mathrm{ddd}, J=5.9,4.2,2.1 \mathrm{~Hz}), 5.80(1 \mathrm{H}$, ddd, $J=5.9,4.0,2.1 \mathrm{~Hz}), 5.03(1 \mathrm{H}, \mathrm{dt}, J=6.7,2.8 \mathrm{~Hz}), 4.20(2 \mathrm{H}, \mathrm{q}, J=7.1 \mathrm{~Hz}), 4.05(1 \mathrm{H}, \mathrm{m}), 2.92(1 \mathrm{H}$, ddd, $J=17.9,6.7,2.0 \mathrm{~Hz}), 2.58(3 \mathrm{H}, \mathrm{s}), 2.52(1 \mathrm{H}, \mathrm{ddd}, J=17.9,5.1,0 \mathrm{~Hz}), 1.31(3 \mathrm{H}, \mathrm{t}, J=7.1 \mathrm{~Hz})$. 


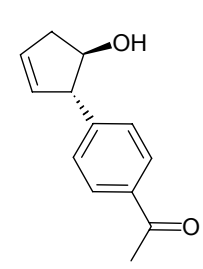

(4c) : (1R, 2S)-trans-2-(4-Acetylphenyl)-cyclopent-3-enol. Obtained by methanolysis of the isolated carbonates mixture (vide supra) and purified by column chromatography (10$25 \% \mathrm{EtOAc} /$ hexanes gradient). The alcohol $\mathbf{4 c}$ was quantitavely isolated as a colourless oil. ${ }^{1} \mathbf{H}$ NMR $\left(400 \mathrm{MHz}, \mathrm{CDCl}_{3}\right): \delta 7.91(2 \mathrm{H}, \mathrm{d}, J=8.3 \mathrm{~Hz}), 7.28(2 \mathrm{H}, \mathrm{d}, J=8.4 \mathrm{~Hz}) 5.95$ $(1 \mathrm{H}, \mathrm{ddd}, J=5.9,4.2,2.0 \mathrm{~Hz}), 5.77(1 \mathrm{H}, \mathrm{ddd}, J=6.0,4.0,2.0 \mathrm{~Hz}), 4.27-4.22(1 \mathrm{H}, \mathrm{m}), 3.84$ $(1 \mathrm{H}$, br. s), $2.83(1 \mathrm{H}$, ddd, $J=17.0,6.8,2.2 \mathrm{~Hz}), 2.59(3 \mathrm{H}, \mathrm{s}), 2.41(1 \mathrm{H}$, dddd, $J=17.0$, 6.2, 4.1, $2.2 \mathrm{~Hz}), 1.85$ (1H, br. s, alcohol). ${ }^{13} \mathrm{C}$ NMR (100 MHz, $\left.\mathrm{CDCl}_{3}\right): \delta 197.9,148.5,135.6,131.4$, 130.3, 128.7, 127.6, 80.7, 60.6, 41.5, 26.6. IR ( NaCl, neat film): 3390 (br), 3056, 3003, 2920, 2844, 1682, 1606, 1560, 1417, 1361, $1273 \mathrm{~cm}^{-1}$. MS m/z (rel. intensity): $202\left(\mathrm{M}^{+}, 57\right), 187$ (100), 173 (37), 159 (17), 131 (62), 115 (35), 91 (28). HRMS (ESI) ${ }^{+}$: Calc'd for $\mathrm{C}_{13} \mathrm{H}_{14} \mathrm{O}_{2}\left[\mathrm{M}^{+}\right]$: 202.099380; found = 202.099888. The ee was determined by HPLC analysis : $88 \%$ ee (Chiralcel OD-H, hexane $/ \mathrm{iPrOH}=96 / 3,1.0 \mathrm{~mL} / \mathrm{min}$ ); $\mathrm{t}_{\mathrm{R}}=39.4 \min (1 S, 2 R)$ minor; $\mathrm{t}_{\mathrm{R}}=49.2 \min (1 R, 2 S)$ major.

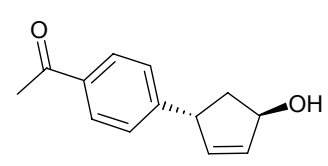

(5c) : (1R, 4R)-trans-4-(4-Acetylphenyl)-cyclopent-2-enol. Colourless oil. ${ }^{1} \mathbf{H}$ NMR $\left(400 \mathrm{MHz}, \mathrm{CDCl}_{3}\right): \delta 7.88(2 \mathrm{H}, \mathrm{d}, J=8.4 \mathrm{~Hz}), 7.22(2 \mathrm{H}, \mathrm{d}, J=8.2 \mathrm{~Hz})$ $6.09(1 \mathrm{H}, \mathrm{ddd}, J=7.5,5.3,2.2 \mathrm{~Hz}), 6.04(1 \mathrm{H}, \mathrm{ddd}, J=7.4,5.7,2.0 \mathrm{~Hz}), 5.09-5.04$ $(1 \mathrm{H}, \mathrm{m}), 4.23-4.19(1 \mathrm{H}, \mathrm{m}), 2.58(3 \mathrm{H}, \mathrm{s}), 2.32(1 \mathrm{H}, \mathrm{ddd}, J=14.3,8.1,2.6 \mathrm{~Hz})$, $2.09(1 \mathrm{H}$, ddd, $J=13.8,6.8,4.5 \mathrm{~Hz}), 1.59\left(1 \mathrm{H}\right.$, br. s, alcohol). ${ }^{13} \mathrm{C}$ NMR $\left(100 \mathrm{MHz}, \mathrm{CDCl}_{3}\right): \delta 197.7$, $150.5,138.2,135.5,134.8,128.8,127.3,110.0,49.9,43.8,26.6$.

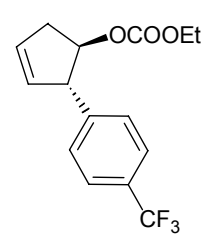

(2d) : (1R, 2S)-trans-2-(4-Trifluoromethyl-phenyl)-cyclopent-3-enyl ethylcarbonate. Prepared according to the general procedure described above. The crude product was purified by column chromatography (dry loaded). The inseparable regioisomers $\mathbf{2}$ and $\mathbf{3}$ were obtained as a colourless oil in $86 \%$ yield ( $42 \mathrm{mg}$ ), showing a 13:1 ratio of $2: 3$ by ${ }^{1} \mathrm{H}$

NMR spectroscopy. The regioisomers were submitted directly to the deprotection conditions and characterized as the free alcohols. Major regioisomer $\mathbf{2 d}{ }^{1} \mathbf{H} \mathbf{N M R}\left(400 \mathrm{MHz}, \mathrm{CDCl}_{3}\right): \delta$ $7.56(2 \mathrm{H}, \mathrm{d}, J=8.1 \mathrm{~Hz}), 7.34(2 \mathrm{H}, \mathrm{d}, J=8.0 \mathrm{~Hz}), 5.99(1 \mathrm{H}, \mathrm{ddd}, J=5.9,4.2,2.1 \mathrm{~Hz}), 5.80(1 \mathrm{H}, \mathrm{ddd}, J=$ 6.0, 4.3, $2.2 \mathrm{~Hz}), 5.01(1 \mathrm{H}, \mathrm{dt}, J=6.7,2.8 \mathrm{~Hz}), 4.21(2 \mathrm{H}, \mathrm{q}, J=7.1 \mathrm{~Hz}), 4.07-4.03(1 \mathrm{H}$, br. s), $2.92(1 \mathrm{H}$, dddd, $J=17.9,6.6,4.1,2.2 \mathrm{~Hz}), 2.56-2.48(1 \mathrm{H}, \mathrm{m}), 1.32(3 \mathrm{H}, \mathrm{t}, J=7.1 \mathrm{~Hz})$.

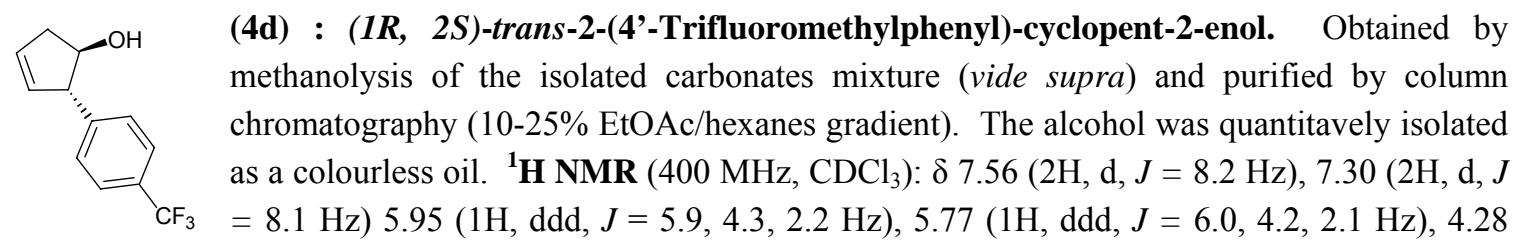
$(1 \mathrm{H}, \mathrm{dt}, J=6.9,4.3 \mathrm{~Hz}), 3.85-3.82(1 \mathrm{H}, \mathrm{m}), 2.82(1 \mathrm{H}, \mathrm{dddd}, J=17.0,6.8,3.9,2.0 \mathrm{~Hz}), 2.40(1 \mathrm{H}$, dddd, $J$ $=17.0,6.1,4.3,1.9 \mathrm{~Hz}), 1.83$ (1H, br. s, alcohol). ${ }^{13} \mathrm{C}$ NMR $\left(100 \mathrm{MHz}, \mathrm{CDCl}_{3}\right): \delta 146.7,131.4,130.4$, 127.7, 125.5 (q, $J=3.8 \mathrm{~Hz}, \mathrm{CF}_{3}$ ), 122.9, 80.8, 60.4, 41.5. IR ( $\mathrm{NaCl}$, neat film): 3324 (br.), 3061, 2922, 2850, 1618, 1418, 1328, 1163, 1126, 1070, $1018 \mathrm{~cm}^{-1}$. MS m/z (rel. intensity): 228 ( $\left.\mathrm{M}^{+}, 98\right), 210$ (30), 172 (100), 159 (52), 131 (20), 115 (19). HRMS (ESI) ${ }^{+}$: Calc'd for $\mathrm{C}_{12} \mathrm{H}_{11} \mathrm{~F}_{3} \mathrm{O}\left[\mathrm{M}^{+}\right]$: 228.07200; found = 228.076170. The ee was determined by HPLC analysis : 88\% ee (Chiralcel OD-H, hexane/iPrOH =98/2, $1.0 \mathrm{~mL} / \mathrm{min}) ; \mathrm{t}_{\mathrm{R}}=14.9 \min (1 S, 2 R)$ minor; $\mathrm{t}_{\mathrm{R}}=17.0 \min (1 R, 2 S)$ major. 


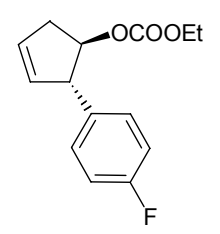

(2e) : (1R, 2S)-trans-2-(4-Fluorophenyl)-cyclopent-3-enyl ethylcarbonate. Prepared according to the general procedure described above. The crude product was purified by column chromatography (dry loaded). The inseparable regioisomers $\mathbf{2}$ and $\mathbf{3}$ were obtained as a colourless oil in $46 \%$ yield $(19 \mathrm{mg})$, showing a $7: 1$ ratio of $2: 3$ by ${ }^{1} \mathrm{H}$ NMR spectroscopy. The regioisomers were submitted directly to the deprotection conditions and characterized as the free alcohols. Major regioisomer $2 \mathbf{2 e}{ }^{1} \mathbf{H} \mathbf{~ N M R}\left(400 \mathrm{MHz}, \mathrm{CDCl}_{3}\right): \delta$ 7.16-7.08 $(2 \mathrm{H}, \mathrm{m}), 6.96-6.89(2 \mathrm{H}, \mathrm{m}), 5.87(1 \mathrm{H}, \mathrm{ddd}, J=6.1,4.2,2.1 \mathrm{~Hz}), 5.72(1 \mathrm{H}, \mathrm{ddd}, J=5.8,4.5,2.3 \mathrm{~Hz}), 4.91$ $(1 \mathrm{H}, \mathrm{dt}, J=6.6,2.7 \mathrm{~Hz}), 4.13(2 \mathrm{H}, \mathrm{q}, J=7.1 \mathrm{~Hz}), 3.93-3.89(1 \mathrm{H}$, br. s), $2.82(1 \mathrm{H}, \mathrm{dddd}, J=17.8,6.3,4.0$, $2.1 \mathrm{~Hz}), 2.41(1 \mathrm{H}, \mathrm{dddd}, J=17.7,5.2,3.7,2.7 \mathrm{~Hz}), 1.31(3 \mathrm{H}, \mathrm{t}, J=7.1 \mathrm{~Hz})$.

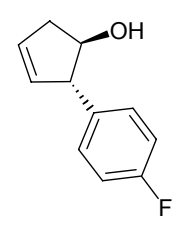

(4e) : (1R, 2S)-trans-2-(4-Fluorophenyl)-cyclopent-3-enol. Obtained by methanolysis of the isolated carbonates mixture (vide supra) and purified by column chromatography (10$25 \%$ EtOAc/hexanes gradient). The alcohol was quantitavely isolated as a colourless oil. ${ }^{1} \mathbf{H}$ NMR $\left(400 \mathrm{MHz}, \mathrm{CDCl}_{3}\right): \delta$ 7.16-7.11 $(2 \mathrm{H}, \mathrm{m}), 7.02-6.96(2 \mathrm{H}, \mathrm{m}) 5.90(1 \mathrm{H}, \mathrm{ddd}, J=6.0$, 4.4, $2.3 \mathrm{~Hz}), 5.75(1 \mathrm{H}, \mathrm{ddd}, J=6.2,4.3,2.1 \mathrm{~Hz}), 4.26-4.21(1 \mathrm{H}, \mathrm{m}), 3.75(1 \mathrm{H}, \mathrm{sp}, J=1.9$ Hz), 2.80 (1H, dddd, $J=16.9,6.6,4.4,2.2 \mathrm{~Hz}), 2.37(1 \mathrm{H}, \mathrm{dsx}, J=16.9,2.1 \mathrm{~Hz}), 1.82(1 \mathrm{H}$, br. s, alcohol). ${ }^{13}$ C NMR $\left(100 \mathrm{MHz}, \mathrm{CDCl}_{3}\right): \delta 162.0(\mathrm{~J}=241 \mathrm{~Hz}), 138.4(\mathrm{~J}=3.3 \mathrm{~Hz}), 132.3,129.9,128.9(\mathrm{~J}=7.8 \mathrm{~Hz})$, $115.5(J=20.9 \mathrm{~Hz}), 81.2,60.1$, 41.6. IR ( $\mathrm{NaCl}$, neat film): 3338 (br.), 3059, 2965, 2852, 1603, 1511, 1417, 1223, 1158, 1054, $950 \mathrm{~cm}^{-1}$. MS m/z (rel. intensity): $178\left(\mathrm{M}^{+}, 67\right), 135$ (41), 122 (100), 109 (60), 57 (32). HRMS (ESI) ${ }^{+}$: Calc'd for $\mathrm{C}_{11} \mathrm{H}_{11} \mathrm{FO}\left[\mathrm{M}^{+}\right]$: 178.079393; found $=178.079724$. The ee was determined by HPLC analysis : 86\% ee (Chiralcel OD-H, hexane/iPrOH $=99 / 1,1.0 \mathrm{~mL} / \mathrm{min}) ; \mathrm{t}_{\mathrm{R}}=28.9 \mathrm{~min}(1 \mathrm{~S}, 2 \mathrm{R})$ minor; $\mathrm{t}_{\mathrm{R}}=30.9 \min (1 R, 2 S)$ major.

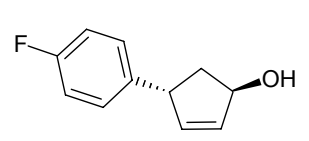

(5e) : (1R, 4R)-trans-2-(4-Fluorophenyl)-cyclopent-2-enol. Colourless oil. ${ }^{1} \mathbf{H}$ NMR $\left(400 \mathrm{MHz}, \mathrm{CDCl}_{3}\right): \delta$ 7.10-7.06 $(2 \mathrm{H}, \mathrm{m}), 7.00-6.94(2 \mathrm{H}, \mathrm{m})$ 6.05-6.00 $(2 \mathrm{H}$, m), 5.08-5.02 $(1 \mathrm{H}, \mathrm{m}), 4.15-4.11(1 \mathrm{H}, \mathrm{m}), 2.28(1 \mathrm{H}, \mathrm{ddd}, J=14.3,8.0,2.8 \mathrm{~Hz})$, $2.05(1 \mathrm{H}$, ddd, $J=14.0,6.9,5.3 \mathrm{~Hz}), 1.30(1 \mathrm{H}$, br. s, alcohol).

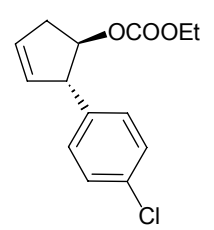

(2f) : (1R, 2S)-trans-2-(4-Chlorophenyl)-cyclopent-3-enyl ethylcarbonate. Prepared according to the general procedure described above. The crude product was purified by column chromatography (dry loaded). The inseparable regioisomers $\mathbf{2}$ and $\mathbf{3}$ were obtained in $53 \%$ yield $\left(23 \mathrm{mg}\right.$ ), showing a 13:1 ratio of $2: 3$ by ${ }^{1} \mathrm{H}$ NMR spectroscopy. The regioisomers were submitted directly to the deprotection conditions and characterized as the free alcohols. Major regioisomer $\mathbf{2 f}:{ }^{1} \mathbf{H}$ NMR $\left(400 \mathrm{MHz}, \mathrm{CDCl}_{3}\right): \delta 7.27(2 \mathrm{H}, \mathrm{d}, J=8.8 \mathrm{~Hz}), 7.16$ $(2 \mathrm{H}, \mathrm{d}, J=8.3 \mathrm{~Hz}), 5.95(1 \mathrm{H}, \mathrm{ddd}, J=5.9,4.3,2.0 \mathrm{~Hz}), 5.78(1 \mathrm{H}, \mathrm{ddd}, J=5.9,4.3,2.0 \mathrm{~Hz}), 4.98(1 \mathrm{H}, \mathrm{dt}, J$ $=6.6,2.8 \mathrm{~Hz}), 4.20(2 \mathrm{H}, \mathrm{q}, J=7.1 \mathrm{~Hz}), 3.99-3.94(1 \mathrm{H}, \mathrm{br} . \mathrm{s}), 2.89(1 \mathrm{H}, \mathrm{dddd}, J=17.8,6.6,4.0,2.2, \mathrm{~Hz})$, 2.49 (1H, dddd, $J=17.8,4.4,2.8,1.2, \mathrm{~Hz}), 1.32(3 \mathrm{H}, \mathrm{t}, J=7.1 \mathrm{~Hz})$.

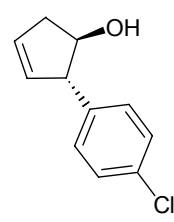

(4f) : (1R, 2S)-trans-2-(4-Chlorophenyl)-cyclopent-3-enol. Obtained by methanolysis of the isolated carbonates mixture (vide supra) and purified by column chromatography (10$25 \%$ EtOAc/hexanes gradient). The alcohol was quantitavely isolated as a colourless oil. ${ }^{1} \mathbf{H}$ NMR (400 MHz, $\left.\mathrm{CDCl}_{3}\right): \delta 7.27(2 \mathrm{H}, \mathrm{d}, J=8.4 \mathrm{~Hz}), 7.11(2 \mathrm{H}, \mathrm{d}, J=8.2 \mathrm{~Hz}) 5.91(1 \mathrm{H}$, ddd, $J=5.9,4.5,2.2 \mathrm{~Hz}), 5.74(1 \mathrm{H}, \mathrm{ddd}, J=6.0,4.2,2.1 \mathrm{~Hz}), 4.23(1 \mathrm{H}, \mathrm{dt}, J=6.7,4.2 \mathrm{~Hz})$, $3.74(1 \mathrm{H}, \mathrm{qn}, J=1.9 \mathrm{~Hz}), 2.80(1 \mathrm{H}$, dddd, $J=17.0,6.8,2.2,1.9 \mathrm{~Hz}), 2.37(1 \mathrm{H}, \mathrm{dddd}, J=17.3,6.0,4.2$, $2.1 \mathrm{~Hz}), 1.84$ (1H, br. s, alcohol). ${ }^{13} \mathrm{C}$ NMR $\left(100 \mathrm{MHz}, \mathrm{CDCl}_{3}\right): \delta 141.0,132.4,131.8,130.0,128.7,80.9$, 
60.0, 41.4. IR ( $\mathrm{NaCl}$, neat film): 3317 (br.), 3057, 2924, 2847, 1651, 1491, 1406, 1318, $1091 \mathrm{~cm}^{-1}$. MS m/z (rel. intensity): 194 ( $\left.\mathrm{M}^{+}, 75\right), 176$ (13), 151 (26), 138 (100), 125 (40), 115 (39), 91 (18). HRMS $(\mathrm{ESI})^{+}$: Calc'd for $\mathrm{C}_{11} \mathrm{H}_{11} \mathrm{ClO}\left[\mathrm{M}^{+}\right]: 194.049843$; found $=194.049690$. The ee was determined by HPLC analysis : $90 \%$ ee (Chiralcel OD-H, hexane $/ \mathrm{PrOH}=99 / 1,1.0 \mathrm{~mL} / \mathrm{min}), \mathrm{t}_{\mathrm{R}}=32.7 \mathrm{~min}(1 \mathrm{~S}, 2 \mathrm{R}), \mathrm{t}_{\mathrm{R}}=36.1$ $\min (1 R, 2 S) .[\alpha]^{26.0}{ }_{589}=-187\left(\mathrm{c} 0.66, \mathrm{CHCl}_{3}\right.$, for $87 \%$ ee).

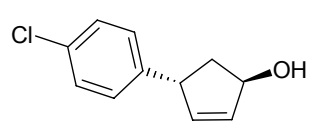

(5f) : (1R, 4R)-trans-2-(4-Chlorophenyl)-cyclopent-2-enol. Colourless oil. ${ }^{1} \mathbf{H}$ NMR $\left(400 \mathrm{MHz}, \mathrm{CDCl}_{3}\right): \delta 7.25(2 \mathrm{H}, \mathrm{d}, J=8.0 \mathrm{~Hz}), 7.06(2 \mathrm{H}, \mathrm{d}, J=8.6 \mathrm{~Hz}) 6.05$ $(1 \mathrm{H}, \mathrm{dt}, J=5.5,2.1 \mathrm{~Hz}), 6.01(1 \mathrm{H}, \mathrm{ddd}, J=5.6,2.0,0.6 \mathrm{~Hz}), 5.04(1 \mathrm{H}, \mathrm{ddd}, J=$ 7.0, 2.0, $0.7 \mathrm{~Hz}), 4.12(1 \mathrm{H}, \mathrm{ddd}, J=7.4,4.1,2.0 \mathrm{~Hz}), 2.28(1 \mathrm{H}, \mathrm{ddd}, J=14.2,8.0,2.6 \mathrm{~Hz}), 2.05(1 \mathrm{H}, \mathrm{ddd}$, $J=14.2,7.0,5.5 \mathrm{~Hz}), 1.60$ (1H, br. s, alcohol).

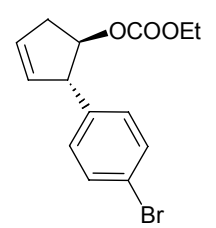

(2g) : (1R, 2S)-trans-2-(4-Bromophenyl)-cyclopent-3-enyl ethylcarbonate. Prepared according to the general procedure described above. The crude product was purified by column chromatography (dry loaded). The inseparable regioisomers $\mathbf{2}$ and $\mathbf{3}$ were obtained in $35 \%$ yield $\left(18 \mathrm{mg}\right.$ ), showing a 10:1 ratio of $2: 3$ by ${ }^{1} \mathrm{H}$ NMR spectroscopy. The regioisomers were submitted directly to the deprotection conditions and characterized as the free alcohols. Major regioisomer $\mathbf{2 g}:{ }^{1} \mathbf{H}$ NMR (400 MHz, $\left.\mathrm{CDCl}_{3}\right): \delta 7.35(2 \mathrm{H}, \mathrm{d}, J=8.4 \mathrm{~Hz}), 7.03$ $(2 \mathrm{H}, \mathrm{d}, J=8.3 \mathrm{~Hz}), 5.88(1 \mathrm{H}, \mathrm{ddd}, J=6.2,4.4,2.3 \mathrm{~Hz}), 5.71(1 \mathrm{H}, \mathrm{ddd}, J=5.9,4.3,2.0 \mathrm{~Hz}), 4.91(1 \mathrm{H}, \mathrm{dt}, J$ $=6.6,2.8 \mathrm{~Hz}), 4.13(2 \mathrm{H}, \mathrm{q}, J=7.1 \mathrm{~Hz}), 3.90-3.85(1 \mathrm{H}$, br. s), $2.82(1 \mathrm{H}$, dddd, $J=17.8,6.6,4.0,2.2, \mathrm{~Hz})$, $2.42(1 \mathrm{H}$, dddd, $J=17.8,2.9,2.0,1.1, \mathrm{~Hz}), 1.24(3 \mathrm{H}, \mathrm{t}, J=7.1 \mathrm{~Hz})$.

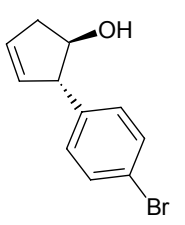

(4g) : (1R, 2S)-trans-2-(4-Bromophenyl)-cyclopent-3-enol. Obtained by methanolysis of the isolated carbonates mixture (vide supra) and purified by column chromatography (10$25 \% \mathrm{EtOAc} /$ hexanes gradient). The alcohol was quantitavely isolated as a colourless oil. ${ }^{1} \mathbf{H}$ NMR $\left(400 \mathrm{MHz}, \mathrm{CDCl}_{3}\right): \delta$ 7.45-7.40 $(2 \mathrm{H}, \mathrm{m}), 7.08-7.04(2 \mathrm{H}, \mathrm{m}) 5.91(1 \mathrm{H}, \mathrm{ddd}, J=6.1$, 4.4, $2.2 \mathrm{~Hz}), 5.74(1 \mathrm{H}, \mathrm{ddd}, J=6.1,3.2,2.1 \mathrm{~Hz}), 4.23(1 \mathrm{H}, \mathrm{dt}, J=6.6,2.2 \mathrm{~Hz}), 3.73(1 \mathrm{H}$, qn, $J=1.9 \mathrm{~Hz}), 2.79(1 \mathrm{H}$, dddd, $J=16.9,6.6,3.9,2.1 \mathrm{~Hz}), 2.37(1 \mathrm{H}$, dsx, $J=17.0,2.0 \mathrm{~Hz}), 1.86(1 \mathrm{H}, \mathrm{br}$. s, alcohol). ${ }^{13} \mathbf{C}$ NMR $\left(100 \mathrm{MHz}, \mathrm{CDCl}_{3}\right): \delta 141.8,132.0,131.9,130.3,129.3,120.6,81.1,60.3,41.6$. IR (NaCl, neat film): 3338 (br.), 3056, 2924, 2848, 1485, 1404, 1315, 1200, 1158, 1072, $1010 \mathrm{~cm}^{-1}$. MS m/z (rel. intensity): 240 (M+, 71), 238 (M+ 73), 222 (6), 220 (6), 184 (98), 182 (100), 171 (26), 169 (27), 141 (24), 128 (51), 116 (68), 91 (28). HRMS (ESI) ${ }^{+}$: Calc'd for $\mathrm{C}_{11} \mathrm{H}_{11} \mathrm{BrO}\left[\mathrm{M}^{+}\right]$: 237.999326; found = 237.999879. The ee was determined by HPLC analysis : $86 \%$ ee (Chiralcel OD-H, hexane/iPrOH $=99 / 1$, $1.0 \mathrm{~mL} / \mathrm{min}) ; \mathrm{t}_{\mathrm{R}}=35.7 \min (1 S, 2 R)$ minor; $\mathrm{t}_{\mathrm{R}}=40.2 \min (1 R, 2 S)$ major.

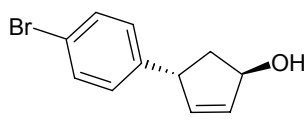

(5g) : (1R, 4R)-trans-2-(4-Bromophenyl)-cyclopent-2-enol. Colourless oil. ${ }^{1} \mathbf{H}$ NMR (400 MHz, $\left.\mathrm{CDCl}_{3}\right): \delta 7.42-7.38(2 \mathrm{H}, \mathrm{m}), 7.03-6.98(2 \mathrm{H}, \mathrm{m}) 6.05(1 \mathrm{H}, \mathrm{dt}, J=$ 5.5, $2.1 \mathrm{~Hz}), 6.00(1 \mathrm{H}, \mathrm{dd}, J=5.6,1.5 \mathrm{~Hz}), 5.07-5.01(1 \mathrm{H}, \mathrm{m}), 4.14-4.07(1 \mathrm{H}, \mathrm{m})$, $2.28(1 \mathrm{H}, \mathrm{ddd}, J=14.1,8.0,2.7 \mathrm{~Hz}), 2.04(1 \mathrm{H}, \mathrm{ddd}, J=14.1,7.0,5.5 \mathrm{~Hz}), 1.51(1 \mathrm{H}$, br. s, alcohol). 


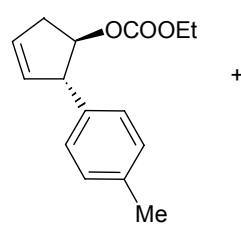

(2h) : (1R, 2S)-trans-2-(4-Methylphenyl)-cyclopent-3-enyl ethylcarbonate. Prepared according to the general procedure described above. The crude product was purified by column chromatography (dry loaded). The inseparable regioisomers $\mathbf{2}$ and $\mathbf{3}$ were obtained in $70 \%$ yield $\left(28 \mathrm{mg}\right.$ ), showing a $20: 1$ ratio of $2: 3$ by ${ }^{1} \mathrm{H}$ NMR spectroscopy. The regioisomers were submitted directly to the deprotection conditions and characterized as the free alcohols. Major regioisomer $\mathbf{2 h}$ : ${ }^{1} \mathbf{H}$ NMR $\left(400 \mathrm{MHz}, \mathrm{CDCl}_{3}\right): \delta 7.20-7.02$ $(4 \mathrm{H}, \mathrm{m}), 5.91(1 \mathrm{H}, \mathrm{ddd}, J=6.0,4.1,2.0 \mathrm{~Hz}), 5.79(1 \mathrm{H}, \mathrm{ddd}, J=5.9,4.2,2.1 \mathrm{~Hz}), 5.00(1 \mathrm{H}, \mathrm{dt}, J=6.1,2.6$ $\mathrm{Hz}), 4.19$ (2H, q, $J=7.1 \mathrm{~Hz}), 3.96$ (1H, br. s), 2.80 (1H, dddd, $J=17.7,6.4,4.0,2.3 \mathrm{~Hz}), 2.47$ (1H, br. d, $J$ $=17.7 \mathrm{~Hz}), 2.32(3 \mathrm{H}, \mathrm{s}), 1.31(3 \mathrm{H}, \mathrm{t}, J=7.1 \mathrm{~Hz})$.

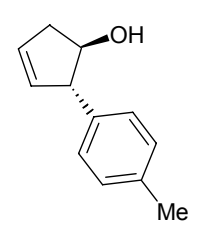

(4h) : (1R, 2S)-trans-2-(4-Methylphenyl)-cyclopent-3-enol. Obtained by methanolysis of the isolated carbonates mixture (vide supra) and purified by column chromatography (10$25 \%$ EtOAc/hexanes gradient). The alcohol was quantitavely isolated as a colourless oil. The characterization data was fully concordant with that already reported in the literature. ${ }^{10 \mathrm{~b}}$ ${ }^{1} \mathbf{H}$ NMR $\left(400 \mathrm{MHz}, \mathrm{CDCl}_{3}\right): \delta 7.12(2 \mathrm{H}, \mathrm{d}, J=7.9 \mathrm{~Hz}), 7.07(2 \mathrm{H}, \mathrm{d}, \mathrm{J}=7.9 \mathrm{~Hz}), 5.89(1 \mathrm{H}$, ddd, $J=6.1,4.4,2.1 \mathrm{~Hz}), 5.76(1 \mathrm{H}, \mathrm{ddd}, J=6.2,4.2,2.1 \mathrm{~Hz}), 4.29-4.24(1 \mathrm{H}, \mathrm{m}), 3.73(1 \mathrm{H}, \mathrm{sp}, J=1.9$ $\mathrm{Hz}), 2.80(1 \mathrm{H}, \mathrm{dddd}, J=16.9,6.2,4.2,2.1 \mathrm{~Hz}), 2.36(1 \mathrm{H}, \mathrm{dsx}, J=16.9,1.9 \mathrm{~Hz}), 1.79(1 \mathrm{H}, \mathrm{d}, J=5.5 \mathrm{~Hz}$, alcohol). ${ }^{13} \mathrm{C}$ NMR (100 MHz, $\left.\mathrm{CDCl}_{3}\right): \delta 142.5,138.2,132.4,129.4,128.5,128.1,127.4,124.4,81.0$, 60.7, 41.3. IR ( $\mathrm{NaCl}$, neat film): 3342 (br.), 3052, 3022, 2920, 2865, 1650, 1492, 1456, 1272, 1159, 1054 $\mathrm{cm}^{-1}$. The ee was determined by HPLC analysis : $84 \%$ ee (Chiralcel OD-H, hexane/iPrOH $=99 / 1,1.0$ $\mathrm{mL} / \mathrm{min}) ; \mathrm{t}_{\mathrm{R}}=23.0 \mathrm{~min}(1 \mathrm{~S}, 2 R)$ minor; $\mathrm{t}_{\mathrm{R}}=28.5 \min (1 R, 2 S)$ major.

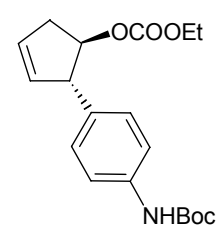

(2i) : (1R, 2S)-trans-2-(4-[t-Butoxycarbonylamino]-phenyl)-cyclopent-3-enyl ethylcarbonate. Prepared according to the general procedure described above. The crude product was purified by column chromatography (dry loaded). The inseparable regioisomers 2 and 3 were obtained in 32\% yield (18 mg), showing a $>20: 1$ ratio of 2:3 by ${ }^{1} \mathrm{H}$ NMR spectroscopy. The regioisomers were submitted directly to the deprotection conditions and characterized as the free alcohols. Major regioisomer $2 \mathbf{i}$ : ${ }^{1} \mathbf{H}$ NMR $\left(400 \mathrm{MHz}, \mathrm{CDCl}_{3}\right)$ : $\delta$ 7.32-7.26 (2H, m), 7.15-7.11 (2H, m), 6.46 (1H, br. s, NHBoc), 5.91 (1H, ddd, J = 6.1, 4.2, $2.1 \mathrm{~Hz}), 5.78$ $(1 \mathrm{H}, \mathrm{ddd}, J=6.1,4.2,2.2 \mathrm{~Hz}), 4.99(1 \mathrm{H}, \mathrm{dt}, J=6.6,3.9 \mathrm{~Hz}), 4.19(2 \mathrm{H}, \mathrm{q}, J=7.1 \mathrm{~Hz}), 3.95(1 \mathrm{H}$, br. s), $2.88(1 \mathrm{H}$, dddd, $J=17.7,6.3,5.0,2.2, \mathrm{~Hz}), 2.46(1 \mathrm{H}, \mathrm{br} . \mathrm{d}, J=17.8 \mathrm{~Hz}), 1.51(9 \mathrm{H}, \mathrm{s}), 1.31(3 \mathrm{H}, \mathrm{t}, J=7.1$ $\mathrm{Hz})$.

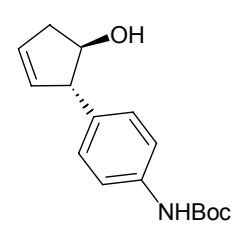

(4i) : (1R, 2S)-trans-2-(4-[t-Butoxycarbonylamino]-phenyl)-cyclopent-3-enol. Obtained by methanolysis of the isolated carbonates mixture (vide supra) and purified by column chromatography (10-25\% EtOAc/hexanes gradient). The alcohol was quantitavely isolated as a colourless oil. ${ }^{1} \mathbf{H}$ NMR $\left(400 \mathrm{MHz}, \mathrm{CDCl}_{3}\right): \delta 7.28(2 \mathrm{H}, \mathrm{d}, J=$ $8.4 \mathrm{~Hz}), 7.10-7.07(2 \mathrm{H}, \mathrm{m}), 6.51(1 \mathrm{H}$, br. s, NHBoc), $5.88(1 \mathrm{H}, \mathrm{ddd}, J=5.9,4.4,2.2 \mathrm{~Hz})$, $5.74(1 \mathrm{H}$, ddd, $J=6.1,4.2,2.1 \mathrm{~Hz}), 4.25-4.20(1 \mathrm{H}, \mathrm{m}), 3.72-3.68(1 \mathrm{H}, \mathrm{m}), 2.77(1 \mathrm{H}$, dddd, $J=16.9,6.5$, 3.9, 1.9, Hz), $2.34(1 \mathrm{H}, \mathrm{d} \mathrm{sx}, J=16.9,2.0 \mathrm{~Hz}), 2.02\left(1 \mathrm{H}, \mathrm{br} . \mathrm{s}\right.$, alcohol), $1.51(9 \mathrm{H}, \mathrm{s}) .{ }^{13} \mathrm{C}$ NMR $(100$ $\left.\mathrm{MHz}, \mathrm{CDCl}_{3}\right): \delta 153.1,137.4,137.1,132.5,129.7,128.0,119.2,81.2,80.7,60.3,41.5,28.6 . \quad$ IR $(\mathrm{NaCl}$, neat film): 3317 (br.), 3055, 2977, 2931, 2858, 1710, 1530, 1315, 1244, 1161, $1055 \mathrm{~cm}^{-1}$. MS m/z (rel. intensity): 275 ( $\left.\mathrm{M}^{+}, 20\right), 219$ (73), 163 (51), 132 (26), 119 (35), 57 (100). HRMS (ESI) ${ }^{+}$: Calc'd for $\mathrm{C}_{16} \mathrm{H}_{21} \mathrm{NO}_{3} \mathrm{Na}\left[\mathrm{M}-\mathrm{Na}^{+}\right]: 298.1413$; found $=298.1408$. The ee was determined by HPLC analysis: $84 \%$ ee (Chiralcel OD-H, hexane/PrOH $=97 / 3,1.0 \mathrm{~mL} / \mathrm{min}) ; \mathrm{t}_{\mathrm{R}}=45.3 \min (1 S, 2 R)$ minor; $\mathrm{t}_{\mathrm{R}}=49.0 \mathrm{~min}(1 R, 2 S)$ major. 


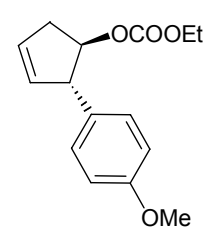

(2j) : (1R, 2S)-trans-2-(4-Methoxyphenyl)-cyclopent-3-enyl ethylcarbonate. Prepared according to the general procedure described above. The crude product was purified by column chromatography (dry loaded). The inseparable regioisomers $\mathbf{2}$ and $\mathbf{3}$ were obtained in $49 \%$ yield $(21 \mathrm{mg})$, showing a $>20: 1$ ratio of $2: 3$ by ${ }^{1} \mathrm{H}$ NMR spectroscopy. The regioisomers were submitted directly to the deprotection conditions and characterized as the free alcohols. Major regioisomer 2j: ${ }^{1} \mathbf{H}$ NMR $\left(400 \mathrm{MHz}, \mathrm{CDCl}_{3}\right): \delta 7.14(2 \mathrm{H}, \mathrm{d}, J=8.5 \mathrm{~Hz}), 6.84$ $(2 \mathrm{H}, \mathrm{d}, J=8.8 \mathrm{~Hz}), 5.92(1 \mathrm{H}, \mathrm{ddd}, J=6.0,4.1,2.0 \mathrm{~Hz}), 5.80(1 \mathrm{H}, \mathrm{ddd}, J=6.2,4.3,2.0 \mathrm{~Hz}), 4.99(1 \mathrm{H}, \mathrm{dt}, J$ $=6.6,2.6 \mathrm{~Hz}), 4.20(2 \mathrm{H}, \mathrm{q}, J=7.1 \mathrm{~Hz}), 3.95(1 \mathrm{H}, \mathrm{br} . \mathrm{s}), 3.79(3 \mathrm{H}, \mathrm{s}), 2.89(1 \mathrm{H}, \mathrm{dddd}, J=17.8,6.3,3.9$, 2.2, Hz), $2.47(1 \mathrm{H}$, dddd, $J=17.8,4.3,2.7,1.1 \mathrm{~Hz}), 1.31(3 \mathrm{H}, \mathrm{t}, J=7.1 \mathrm{~Hz})$.

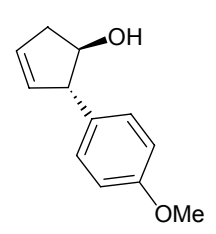

(4j) : (1R, 2S)-trans-2-(4-Methoxyphenyl)-cyclopent-3-enol. Obtained by methanolysis of the isolated carbonates mixture (vide supra) and purified by column chromatography (10-25\% EtOAc/hexanes gradient). The alcohol was quantitavely isolated as a colourless oil. The characterization data was fully concordant with that already reported in the literature. ${ }^{10 \mathrm{~b}}{ }^{1} \mathbf{H}$ NMR $\left(400 \mathrm{MHz}, \mathrm{CDCl}_{3}\right): \delta 7.10(2 \mathrm{H}, \mathrm{d}, J=8.8 \mathrm{~Hz}), 6.85(2 \mathrm{H}, \mathrm{d}, J=8.7$ Hz) $5.88(1 \mathrm{H}, \mathrm{ddd}, J=6.3,4.4,2.3 \mathrm{~Hz}), 5.76(1 \mathrm{H}, \mathrm{ddd}, J=6.0,4.2,2.1 \mathrm{~Hz}), 4.24(1 \mathrm{H}, \mathrm{dt}, J=6.7,4.1 \mathrm{~Hz})$, $3.79(3 \mathrm{H}, \mathrm{s}), 3.72(1 \mathrm{H}, \mathrm{qn}, J=1.9 \mathrm{~Hz}), 2.79(1 \mathrm{H}, \mathrm{dddd}, J=16.9,6.7,3.9,1.8 \mathrm{~Hz}), 2.35(1 \mathrm{H}$, dddd, $J=$ 16.9, 6.3, 4.2, $1.9 \mathrm{~Hz}), 1.85$ (1H, br. s, alcohol). ${ }^{13} \mathrm{C}$ NMR (100 MHz, $\left.\mathrm{CDCl}_{3}\right): \delta 158.4,134.5,132.5$, 129.2, 128.3, 114.0, 81.0, 59.9, 55.3, 41.2. IR (NaCl, neat film): 3348 (br.), 3055, 3001, 2931, 2907, 2838, 1651, 1614, 1539, 1511, 1459, 1250, $1179 \mathrm{~cm}^{-1}$. MS m/z (rel. intensity): $190\left(\mathrm{M}^{+}, 61\right), 172$ (8), 147 (45), 134 (100), 121 (44), 115 (21), 91 (29). HRMS (ESI) ${ }^{+}$: Calc'd for $\mathrm{C}_{12} \mathrm{H}_{14} \mathrm{O}_{2}\left[\mathrm{M}^{+}\right]$: 190.099380; found = 190.099729. The ee was determined by HPLC analysis : 89\% ee (Chiralcel OD-H, hexane/iPrOH $=99 / 1$, $1.0 \mathrm{~mL} / \mathrm{min}) ; \mathrm{t}_{\mathrm{R}}=48.9 \min (1 S, 2 R)$ minor; $\mathrm{t}_{\mathrm{R}}=50.6 \min (1 R, 2 S)$ major.

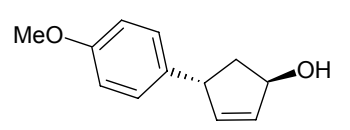

(5j) : (1R, 4R)-trans-4-(4-Methoxyphenyl)-cyclopent-2-enol. Colourless oil. The characterization data was fully concordant with that already reported in the literature. ${ }^{10 \mathrm{~b}}{ }^{1} \mathbf{H}$ NMR $\left(400 \mathrm{MHz}, \mathrm{CDCl}_{3}\right): \delta 7.04(2 \mathrm{H}, \mathrm{d}, J=8.8 \mathrm{~Hz}), 6.83(2 \mathrm{H}$, $\mathrm{d}, J=8.7 \mathrm{~Hz}) 6.01(1 \mathrm{H}, \mathrm{dt}, J=1.9,1.6 \mathrm{~Hz}), 5.06-5.01(1 \mathrm{H}, \mathrm{m}), 4.12-4.08(1 \mathrm{H}, \mathrm{m}), 3.79(3 \mathrm{H}, \mathrm{s}), 2.26(1 \mathrm{H}$, ddd, $J=15.1,8.0,2.6 \mathrm{~Hz}), 2.06(1 \mathrm{H}$, ddd, $J=14.0,6.9,5.5 \mathrm{~Hz})$.

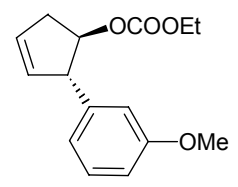

(2k) : (1R, 2S)-trans-2-(3-Methoxyphenyl)-cyclopent-3-enyl ethylcarbonate. Prepared according to the general procedure described above. The crude product was purified by column chromatography (dry loaded). The inseparable regioisomers $\mathbf{2}$ and $\mathbf{3}$ were obtained in $63 \%$ yield $(27 \mathrm{mg})$, showing a $>20: 1$ ratio of $2: 3$ by ${ }^{1} \mathrm{H}$ NMR spectroscopy. The regioisomers were submitted directly to the deprotection conditions and characterized as the free alcohols. Major regioisomer 2k: ${ }^{1} \mathbf{H}$ NMR (400 MHz, $\left.\mathrm{CDCl}_{3}\right)$ : $\delta$ 7.19-7.12 (1H, m), 6.77-6.69 $(3 \mathrm{H}, \mathrm{m}), 5.86(1 \mathrm{H}, \mathrm{ddd}, J=5.9,4.1,2.0 \mathrm{~Hz}), 5.73(1 \mathrm{H}, \mathrm{ddd}, J=5.9,4.2,2.0 \mathrm{~Hz}), 4.97(1 \mathrm{H}, \mathrm{dt}, J=6.6,2.8$ $\mathrm{Hz}), 4.12(2 \mathrm{H}, \mathrm{q}, J=7.1 \mathrm{~Hz}), 3.93-3.88(1 \mathrm{H}, \mathrm{m}), 3.72(3 \mathrm{H}, \mathrm{s}), 2.84(1 \mathrm{H}, \mathrm{dddd}, J=17.8,6.3,4.0,2.2 \mathrm{~Hz})$, 2.45-2.36 (1H, m), $1.24(3 \mathrm{H}, \mathrm{t}, J=7.1 \mathrm{~Hz})$.

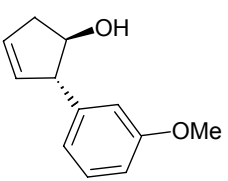

(4k) : (1R, 2S)-trans-2-(3-Methoxyphenyl)-cyclopent-3-enol. Obtained by methanolysis of the isolated carbonates mixture (vide supra) and purified by column chromatography (10-25\% EtOAc/hexanes gradient). The alcohol was quantitavely isolated as a colourless oil. The characterization data was fully concordant with that already reported in the literature. ${ }^{10 \mathrm{~b}}{ }^{1} \mathbf{H}$ NMR $\left(400 \mathrm{MHz}, \mathrm{CDCl}_{3}\right): \delta 7.13(1 \mathrm{H}, \mathrm{t}, \mathrm{J}=7.4), 6.70-6.62(3 \mathrm{H}, \mathrm{m})$ $5.80(1 \mathrm{H}, \mathrm{ddd}, J=6.0,4.4,2.2 \mathrm{~Hz}), 5.67(1 \mathrm{H}, \mathrm{ddd}, J=6.1,4.1,2.1 \mathrm{~Hz}), 4.18(1 \mathrm{H}, \mathrm{dt}, J=6.7,4.2 \mathrm{~Hz}), 3.70$ 
(3H, s), $3.64(1 \mathrm{H}, \mathrm{sp}, J=1.9 \mathrm{~Hz}), 2.70(1 \mathrm{H}, \mathrm{dddd}, J=16.9,6.7,3.9,2.1 \mathrm{~Hz}), 2.27(1 \mathrm{H}, \mathrm{dsx}, J=16.9,2.1$ $\mathrm{Hz}), 1.80$ (1H, br. s, alcohol). ${ }^{13} \mathrm{C}$ NMR $\left(100 \mathrm{MHz}, \mathrm{CDCl}_{3}\right): \delta 160.1,144.3,132.2,129.8,128.7,120.1$, 113.2, 112.1, 81.2, 60.8, 55.3, 41.6. IR ( NaCl, neat film): 3323 (br.), 3055, 3023, 2909, 2842, 1588, 1484, 1406, 1073, $1010 \mathrm{~cm}^{-1}$. MS m/z (rel. intensity): 190 (M+100), 172 (20), 162 (50), 147 (41), 134 (63), 129 (27), 121 (38), 115 (32), 91 (32). HRMS (ESI) ${ }^{+}$: Calc'd for $\mathrm{C}_{12} \mathrm{H}_{14} \mathrm{O}_{2}\left[\mathrm{M}^{+}\right]$: 190.099380; found = 190.099915. The ee was determined by HPLC analysis : $92 \%$ ee (Chiralcel OD-H, hexane/iPrOH $=98 / 2$ to $96 / 4$ gradient, $1.0 \mathrm{~mL} / \mathrm{min}) ; \mathrm{t}_{\mathrm{R}}=35.5 \mathrm{~min}(1 S, 2 R)$ minor; $\mathrm{t}_{\mathrm{R}}=50.1 \mathrm{~min}(1 R, 2 S)$ major.

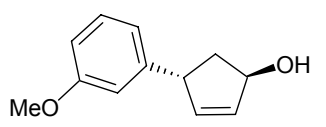

(5k) : (1R, 4R)-trans-4-(3-Methoxyphenyl)-cyclopent-2-enol. Colourless oil. The characterization data was fully concordant with that already reported in the literature. ${ }^{10 \mathrm{~b}}{ }^{1} \mathbf{H}$ NMR $\left(400 \mathrm{MHz}, \mathrm{CDCl}_{3}\right): \delta 7.21(2 \mathrm{H}, \mathrm{t}, J=7.9 \mathrm{~Hz}), 6.77-6.67$ $(3 \mathrm{H}, \mathrm{m})$ 6.06-6.01 $(2 \mathrm{H}, \mathrm{m}), 5.08-5.02(1 \mathrm{H}, \mathrm{m}), 4.15-4.10(1 \mathrm{H}, \mathrm{m}), 3.79(3 \mathrm{H}, \mathrm{s}), 2.28(1 \mathrm{H}, \mathrm{ddd}, J=14.1$, $8.0,2.8 \mathrm{~Hz}), 2.11(1 \mathrm{H}, \mathrm{ddd}, J=14.1,6.9,6.0 \mathrm{~Hz}), 1.48(1 \mathrm{H}$, br. s).

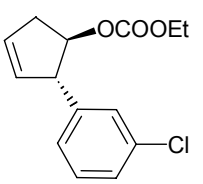

(2l) : (1R, 2S)-trans-2-(3-Chlorophenyl)-cyclopent-3-enyl ethylcarbonate. Prepared according to the general procedure described above. The crude product was purified by column chromatography (dry loaded). The inseparable regioisomers $\mathbf{2}$ and $\mathbf{3}$ were obtained in $87 \%$ yield $\left(38 \mathrm{mg}\right.$ ), showing a $10: 1$ ratio of $2: 3$ by ${ }^{1} \mathrm{H}$ NMR spectroscopy. The regioisomers were submitted directly to the deprotection conditions and characterized as the free alcohols. Major regioisomer 2l: ${ }^{1} \mathbf{H}$ NMR $\left(400 \mathrm{MHz}, \mathrm{CDCl}_{3}\right): \delta 7.26-7.11(4 \mathrm{H}, \mathrm{m}), 5.96(1 \mathrm{H}, \mathrm{ddd}, J=6.1,4.2,2.1$ $\mathrm{Hz}), 5.77(1 \mathrm{H}, \mathrm{ddd}, J=6.2,4.3,2.0 \mathrm{~Hz}), 5.00(1 \mathrm{H}, \mathrm{dt}, J=6.6,2.8 \mathrm{~Hz}), 4.20(2 \mathrm{H}, \mathrm{q}, J=7.1 \mathrm{~Hz}), 3.99-3.96$ $(1 \mathrm{H}, \mathrm{m}), 2.91(1 \mathrm{H}$, dddd, $J=17.8,6.4,4.0,2.3 \mathrm{~Hz}), 2.53-2.45(1 \mathrm{H}, \mathrm{m}), 1.31(3 \mathrm{H}, \mathrm{t}, J=7.1 \mathrm{~Hz})$.

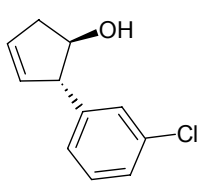

(4I) : (1R, 2S)-trans-2-(3-Chlorophenyl)-cyclopent-3-enol. Obtained by methanolysis of the isolated carbonates mixture (vide supra) and purified by column chromatography (10$25 \% \mathrm{EtOAc} /$ hexanes gradient). The alcohol was quantitavely isolated as a colourless oil. ${ }^{1} \mathbf{H}$ NMR $\left(400 \mathrm{MHz}, \mathrm{CDCl}_{3}\right): \delta$ 7.26-7.15 $(3 \mathrm{H}, \mathrm{m}), 7.08-7.05(1 \mathrm{H}, \mathrm{m}) 5.92(1 \mathrm{H}, \mathrm{ddd}, J=$ 6.3, 4.4, $2.2 \mathrm{~Hz}), 5.74(1 \mathrm{H}, \mathrm{ddd}, J=6.0,4.4,2.1 \mathrm{~Hz}), 4.25(1 \mathrm{H}, \mathrm{dt}, J=6.8,4.1 \mathrm{~Hz}), 3.73(1 \mathrm{H}, \mathrm{sp}, J=1.9$ Hz), 2.80 (1H, dddd, $J=17.0,6.8,3.9,1.7 \mathrm{~Hz}), 2.37$ (1H, dddd, $J=17.0,6.5,4.2,2.1 \mathrm{~Hz}), 2.07$ (1H, br. s, alcohol). ${ }^{13} \mathrm{C}$ NMR (100 MHz, $\left.\mathrm{CDCl}_{3}\right): \delta 144.7,134.4,131.5,130.2,129.8,127.4,126.8,125.6,80.8$, 60.3, 41.4. IR ( $\mathrm{NaCl}$, neat film): 3323 (br.), 3059, 2931, 2906, 2841, 1596, 1572, 1478, 1426, 1195, 1078 $\mathrm{cm}^{-1}$. MS m/z (rel. intensity): $194\left(\mathrm{M}^{+}, 100\right), 176$ (20), 166 (32), 151 (25), 141 (47), 138 (91), 128 (44), 125 (42), 115 (49), 91 (21). HRMS (ESI) ${ }^{+}$: Calc'd for $\mathrm{C}_{11} \mathrm{H}_{11} \mathrm{ClO}\left[\mathrm{M}^{+}\right]$: 194.049843; found = 194.049579. The ee was determined by HPLC analysis : $90 \%$ ee (Chiralcel OD-H, hexane/iPrOH $=99 / 1,1.0 \mathrm{~mL} / \mathrm{min}$ ); $\mathrm{t}_{\mathrm{R}}=31.8 \min (1 S, 2 R)$ minor; $\mathrm{t}_{\mathrm{R}}=40.4 \min (1 R, 2 S)$ major.

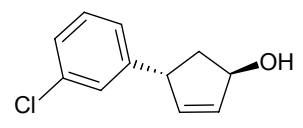

(5l) : (1R, 4R)-trans-4-(3-Chlorophenyl)-cyclopent-2-enol. Colourless oil. ${ }^{1} \mathbf{H}$ NMR $\left(400 \mathrm{MHz}, \mathrm{CDCl}_{3}\right): \delta$ 7.26-7.16 $(2 \mathrm{H}, \mathrm{m}), 7.11-7.10(1 \mathrm{H}, \mathrm{m}), 7.03-7.00(1 \mathrm{H}$, m) $6.06(1 \mathrm{H}, \mathrm{dt}, J=5.5,2.2 \mathrm{~Hz}), 6.00(1 \mathrm{H}, \mathrm{dd}, J=5.6,2.0, \mathrm{~Hz}), 5.07-5.03(1 \mathrm{H}, \mathrm{m})$, $4.12(1 \mathrm{H}$, dddd, $J=9.8,7.6,4.1,2.0 \mathrm{~Hz}), 2.29(1 \mathrm{H}, \mathrm{ddd}, J=14.1,8.0,2.7 \mathrm{~Hz}), 2.07(1 \mathrm{H}, \mathrm{ddd}, J=14.2$, 7.0, $5.5 \mathrm{~Hz}), 1.58\left(1 \mathrm{H}\right.$, br. s, alcohol). ${ }^{13} \mathrm{C}$ NMR (100 MHz, $\left.\mathrm{CDCl}_{3}\right): \delta 146.9,138.3,134.7,134.4,129.8$, $127.2,126.5,125.3,77.3,49.6,43.9$.

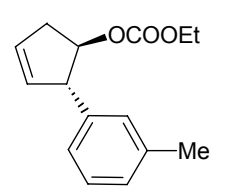

(2m) : (1R, 2S)-trans-2-(3-Methylphenyl)-cyclopent-3-enyl ethylcarbonate. Prepared according to the general procedure described above. The crude product was purified by column chromatography (dry loaded). The inseparable regioisomers $\mathbf{2}$ and $\mathbf{3}$ were obtained 
in $78 \%$ yield (32 mg), showing a 20:1 ratio of $2: 3$ by ${ }^{1} \mathrm{H}$ NMR spectroscopy. The regioisomers were submitted directly to the deprotection conditions and characterized as the free alcohols. Major regioisomer 2m: ${ }^{1} \mathbf{H}$ NMR (400 MHz, $\left.\mathrm{CDCl}_{3}\right): \delta 7.22-7.01(4 \mathrm{H}, \mathrm{m}), 5.92(1 \mathrm{H}$, ddd, $J=5.9,4.0,2.0 \mathrm{~Hz})$, $5.80(1 \mathrm{H}, \mathrm{ddd}, J=5.9,4.4,2.1 \mathrm{~Hz}), 5.04(1 \mathrm{H}, \mathrm{dt}, J=5.9,2.7 \mathrm{~Hz}), 4.19(2 \mathrm{H}, \mathrm{q}, J=7.1 \mathrm{~Hz}), 3.98-3.94(1 \mathrm{H}$, m), $2.92(1 \mathrm{H}$, dddd, $J=17.7,6.4,4.0,2.3 \mathrm{~Hz}), 2.51-2.43(1 \mathrm{H}, \mathrm{m}), 2.33(3 \mathrm{H}, \mathrm{s}), 1.31(3 \mathrm{H}, \mathrm{t}, J=7.1 \mathrm{~Hz})$.

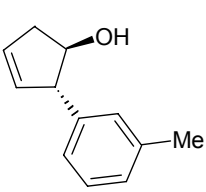

(4m) : (1R, 2S)-trans-2-(3-Methylphenyl)-cyclopent-3-enol. Obtained by methanolysis of the isolated carbonates mixture (vide supra) and purified by column chromatography (10-25\% EtOAc/hexanes gradient). The alcohol was quantitavely isolated as a colourless oil. The characterization data was fully concordant with that reported. ${ }^{10 \mathrm{~b}}{ }^{\mathbf{1}} \mathbf{H}$ NMR (400 $\left.\mathrm{MHz}, \mathrm{CDCl}_{3}\right): \delta 7.21(1 \mathrm{H}, \mathrm{t}, J=7.5 \mathrm{~Hz}), 7.06-6.97(3 \mathrm{H}, \mathrm{m}), 5.89(1 \mathrm{H}, \mathrm{ddd}, J=5.0,4.4,2.2 \mathrm{~Hz}), 5.77(1 \mathrm{H}$, ddd, $J=6.0,4.2,2.1 \mathrm{~Hz}), 4.31-4.26(1 \mathrm{H}, \mathrm{m}), 3.73(1 \mathrm{H}, \mathrm{sp}, J=1.9 \mathrm{~Hz}), 2.81(1 \mathrm{H}$, dddd, $J=16.9,6.5,4.1$, $2.0 \mathrm{~Hz}), 2.37(1 \mathrm{H}, \mathrm{dddd}, J=16.9,6.3,4.1,2.0 \mathrm{~Hz}), 2.34(3 \mathrm{H}, \mathrm{s}), 1.88$ (1H, br. s, alcohol). ${ }^{13} \mathrm{C}$ NMR (100 $\left.\mathrm{MHz}, \mathrm{CDCl}_{3}\right): \delta 142.5,138.2,132.4,129.4,128.5,128.1,127.4,124.4,81.0,60.7,41.3$. IR $(\mathrm{NaCl}$, neat film): 3323 (br.), 3336 (br.), 3056, 3022, 2921, 2863, 1650, 1607, 1490, 1456, 1272, 1161, $1054 \mathrm{~cm}^{-1}$. MS m/z (rel. intensity): 174 ( $\left.\mathrm{M}^{+}, 93\right), 156$ (25), 146 (55), 131 (69), 128 (47), 118 (100), 115 (41), 105 (50), 91 (42). HRMS (ESI) ${ }^{+}: \mathrm{Calc}^{\prime} \mathrm{d}$ for $\mathrm{C}_{12} \mathrm{H}_{14} \mathrm{O}\left[\mathrm{M}^{+}\right]: 174.104465$; found $=174.104256$. The ee was determined by HPLC analysis : 92\% ee (Chiralcel OD-H, hexane/iPrOH $=99 / 1,1.0 \mathrm{~mL} / \mathrm{min}) ; \mathrm{t}_{\mathrm{R}}=20.4 \mathrm{~min}(1 S, 2 R)$ minor; $\mathrm{t}_{\mathrm{R}}=26.4 \min (1 R, 2 S)$ major.

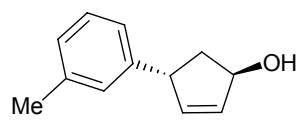

(5m) : (1R, 4R)-trans-4-(3-Methylphenyl)-cyclopent-2-enol. Colourless oil. The characterization data was fully concordant with that already reported in the literature. ${ }^{10 \mathrm{~b}}{ }^{1} \mathbf{H}$ NMR $\left(400 \mathrm{MHz}, \mathrm{CDCl}_{3}\right): \delta 7.18(1 \mathrm{H}, \mathrm{t}, J=8.2 \mathrm{~Hz}), 7.01(1 \mathrm{H}, \mathrm{d}, J$ = 7.7 Hz), 6.95-6.92 $(2 \mathrm{H}, \mathrm{m})$ 6.06-6.01 $(2 \mathrm{H}, \mathrm{m}), 5.07-5.03(1 \mathrm{H}, \mathrm{m}), 4.13-4.08(1 \mathrm{H}, \mathrm{m}), 2.33(3 \mathrm{H}, \mathrm{s}), 2.27$ $(1 \mathrm{H}, \mathrm{ddd}, J=14.1,8.0,2.6 \mathrm{~Hz}), 2.10(1 \mathrm{H}, \mathrm{ddd}, J=14.1,7.0,5.5 \mathrm{~Hz}), 1.56\left(1 \mathrm{H}\right.$, br. s, alcohol). ${ }^{13} \mathrm{C}$ NMR $\left(100 \mathrm{MHz}, \mathrm{CDCl}_{3}\right): \delta$ 144.7, 139.3, 138.2, 133.9, 128.5, 127.8, 127.1, 124.1, 77.5, 49.8, 44.1, 21.4.

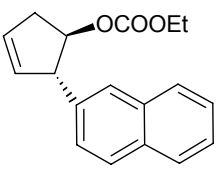

(2n) : (1R, 2S)-trans-2-(2-Naphthalenyl)- cyclopent-3-enyl ethylcarbonate. Prepared according to the general procedure described above. The crude product was purified by column chromatography (dry loaded). The inseparable regioisomers 2 and $\mathbf{3}$ were obtained as a colourless oil in 78\% yield (36 mg), showing a $>20: 1$ ratio of $2: 3$ by ${ }^{1} \mathrm{H}$

NMR spectroscopy. The regioisomers were submitted directly to the deprotection conditions and characterized as the free alcohols. Major regioisomer $\mathbf{2 n}$ : ${ }^{1} \mathbf{H} \mathbf{~ N M R}\left(400 \mathrm{MHz}, \mathrm{CDCl}_{3}\right): \delta 7.86-7.77(3 \mathrm{H}$, m), $7.62(1 \mathrm{H}, \mathrm{s}), 7.49-7.39(3 \mathrm{H}, \mathrm{m}), 6.01(1 \mathrm{H}, \mathrm{ddd}, J=6.1,4.0,2.1 \mathrm{~Hz}), 5.90(1 \mathrm{H}, \mathrm{ddd}, J=5.8,4.0,2.0$ $\mathrm{Hz}), 5.12(1 \mathrm{H}, \mathrm{dt}, J=6.0,2.7 \mathrm{~Hz}), 4.21(2 \mathrm{H}, \mathrm{q}, J=7.1 \mathrm{~Hz}), 4.19-4.16(1 \mathrm{H}, \mathrm{m}), 2.97(1 \mathrm{H}$, dddd, $J=17.8$, $6.2,4.4,2.5 \mathrm{~Hz}), 2.57-2.49(1 \mathrm{H}, \mathrm{m}), 1.32(3 \mathrm{H}, \mathrm{t}, J=7.1 \mathrm{~Hz})$.

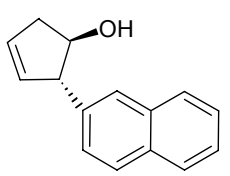

(4n) : (1R, 2S)-trans-2-(2-Naphthalenyl)-cyclopent-3-enol. Obtained by methanolysis of the isolated carbonates mixture (vide supra) and purified by column chromatography (10-25\% EtOAc/hexanes gradient). The alcohol was quantitavely isolated as a colourless oil. ${ }^{1} \mathbf{H}$ NMR $\left(400 \mathrm{MHz}, \mathrm{CDCl}_{3}\right): \delta 7.83-7.78(3 \mathrm{H}, \mathrm{m}), 7.62(1 \mathrm{H}, \mathrm{s}), 7.49-$ $7.42(2 \mathrm{H}, \mathrm{m}), 7.33(1 \mathrm{H}, \mathrm{dd}, J=8.5,1.7 \mathrm{~Hz}), 5.97(1 \mathrm{H}, \mathrm{ddd}, J=6.0,4.4,2.2 \mathrm{~Hz}), 5.87(1 \mathrm{H}, \mathrm{ddd}, J=6.2$, 4.2, $2.1 \mathrm{~Hz}), 4.37(1 \mathrm{H}, \mathrm{dt}, J=6.7,4.1 \mathrm{~Hz}), 3.94(3 \mathrm{H}, \mathrm{sp}, J=1.9 \mathrm{~Hz}), 2.86(1 \mathrm{H}, \mathrm{dddd}, J=16.9,6.3,3.9,2.0$ $\mathrm{Hz}), 2.42(1 \mathrm{H}, \mathrm{dddd}, J=17.0,6.0,3.9,2.0 \mathrm{~Hz}), 1.99\left(1 \mathrm{H}\right.$, br. s, alcohol). ${ }^{13} \mathrm{C}$ NMR $\left(100 \mathrm{MHz}, \mathrm{CDCl}_{3}\right): \delta$ $139.9,133.5,132.5,132.2,129.8,128.3,127.6,126.1,126.0,125.5,125.4,80.8,60.9,41.4$. IR $(\mathrm{NaCl}$, neat film): 3028 (br.), 3054, 3017, 2925, 2854, 1650, 1633, 1506, 1456, 1271, 1216, $1050 \mathrm{~cm}^{-1}$. MS m/z (rel. intensity): 210 ( $\left.\mathrm{M}^{+}, 100\right), 192$ (24), 179 (46), 165 (58), 154 (96), 141 (48), 128 (36), 115 (19). HRMS 
$(\mathrm{ESI})^{+}$: Calc'd for $\mathrm{C}_{15} \mathrm{H}_{14} \mathrm{O}\left[\mathrm{M}^{+}\right]: 210.104465$; found $=210.105000$. The ee was determined by HPLC analysis : $90 \%$ ee (Chiralcel OD-H, hexane $/ \mathrm{iPrOH}=98 / 2,1.0 \mathrm{~mL} / \mathrm{min}) ; \mathrm{t}_{\mathrm{R}}=34.3 \mathrm{~min}(1 \mathrm{~S}, 2 \mathrm{R})$ minor; $\mathrm{t}_{\mathrm{R}}=$ $51.8 \min (1 R, 2 S)$ major.

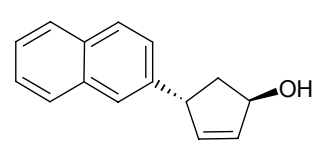

(5n) : (1R, 4R)-trans-4-(2-Naphthalenyl)-cyclopent-2-enol. Colourless oil. ${ }^{1} \mathbf{H}$ NMR $\left(400 \mathrm{MHz}, \mathrm{CDCl}_{3}\right): \delta$ 7.81-7.76 $(3 \mathrm{H}, \mathrm{m}), 7.58(1 \mathrm{H}, \mathrm{s}), 7.44(2 \mathrm{H}, \mathrm{ddd}, J=$ $13.1,6.9,1.6 \mathrm{~Hz}), 7.26-7.23(1 \mathrm{H}, \mathrm{m}), 6.13(1 \mathrm{H}, \mathrm{dd}, J=5.5,2.0 \mathrm{~Hz}), 6.10(1 \mathrm{H}, \mathrm{dt}, J$ = 5.5, $2.2 \mathrm{~Hz}), 5.13-5.08(1 \mathrm{H}, \mathrm{m}), 4.35-4.30(1 \mathrm{H}, \mathrm{m}), 2.35(1 \mathrm{H}, \mathrm{ddd}, J=14.3,8.2$, $2.8 \mathrm{~Hz}), 2.20(1 \mathrm{H}$, ddd, $J=14.2,6.8,5.5 \mathrm{~Hz}), 1.71\left(1 \mathrm{H}\right.$, br. s, alcohol). ${ }^{13} \mathrm{C}$ NMR $\left(100 \mathrm{MHz}, \mathrm{CDCl}_{3}\right): \delta$ $142.1,139.0,134.3,133.5,132.3,128.3,127.6,127.5,126.1,125.7,125.4,125.2,77.6,50.1,44.0$.

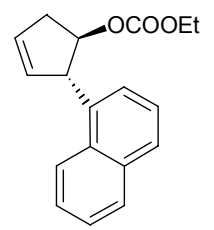

(2o) : (1R, 2S)-trans-2-(1-Naphthalenyl)- cyclopent-3-enyl ethylcarbonate. Prepared according to the general procedure described above. The crude product was purified by column chromatography (dry loaded). The inseparable regioisomers $\mathbf{2}$ and $\mathbf{3}$ were obtained in $50 \%$ yield $\left(23 \mathrm{mg}\right.$ ), showing a $1: 1$ ratio of $2: 3$ by ${ }^{1} \mathrm{H}$ NMR spectroscopy. The regioisomers were submitted directly to the deprotection conditions and characterized as the free alcohols. Major regioisomer 2o: ${ }^{1} \mathbf{H}$ NMR $\left(400 \mathrm{MHz}, \mathrm{CDCl}_{3}\right): \delta 8.18(1 \mathrm{H}, \mathrm{d}, J=8.2 \mathrm{~Hz}), 7.87$ $(1 \mathrm{H}, \mathrm{dd}, J=8.0,1.5 \mathrm{~Hz}), 7.76(1 \mathrm{H}, \mathrm{d}, J=8.2 \mathrm{~Hz}), 7.56(1 \mathrm{H}, \mathrm{dt}, J=6.9,1.6 \mathrm{~Hz}), 7.50(1 \mathrm{H}, \mathrm{dt}, J=6.9,1.5$ $\mathrm{Hz}), 7.40(1 \mathrm{H}, \mathrm{t}, J=8.2 \mathrm{~Hz}), 7.26(1 \mathrm{H}, \mathrm{s}), 6.04(1 \mathrm{H}, \mathrm{ddd}, J=5.9,4.0,2.0 \mathrm{~Hz}), 5.94(1 \mathrm{H}, \mathrm{ddd}, J=5.9,4.3$, $2.0 \mathrm{~Hz}), 5.18(1 \mathrm{H}, \mathrm{dt}, J=6.5,2.4 \mathrm{~Hz}), 4.80(1 \mathrm{H}$, br. s), $4.18(2 \mathrm{H}, \mathrm{q}, J=7.1 \mathrm{~Hz}), 2.97(1 \mathrm{H}$, dddd, $J=17.9$, 6.4, 4.2, $2.1 \mathrm{~Hz}), 2.54(1 \mathrm{H}$, dddd, $J=17.9,4.1,2.5,1.0 \mathrm{~Hz}), 1.31(3 \mathrm{H}, \mathrm{t}, J=7.1 \mathrm{~Hz})$.

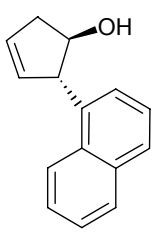

(40) : (1R, 2S)-trans-2-(1-Naphthalenyl)-cyclopent-3-enol. Obtained by methanolysis of the isolated carbonates mixture (vide supra) and purified by column chromatography (10-25\% EtOAc/hexanes gradient). The alcohol was quantitavely isolated as a colourless oil. ${ }^{1} \mathbf{H}$ NMR $\left(400 \mathrm{MHz}, \mathrm{CDCl}_{3}\right): \delta 8.33(1 \mathrm{H}, \mathrm{d}, J=8.4 \mathrm{~Hz}), 7.88(1 \mathrm{H}, \mathrm{d}, J=7.9 \mathrm{~Hz}), 7.75(1 \mathrm{H}, \mathrm{d}, J$ $=8.2 \mathrm{~Hz}), 7.58(1 \mathrm{H}, \mathrm{dt}, J=6.9,1.2 \mathrm{~Hz}), 7.52(1 \mathrm{H}, \mathrm{dt}, J=6.8,1.0 \mathrm{~Hz}), 7.39(1 \mathrm{H}, \mathrm{t}, J=8.0$ $\mathrm{Hz}), 7.22(1 \mathrm{H}, \mathrm{d}, J=8.0 \mathrm{~Hz}), 6.06(1 \mathrm{H}, \mathrm{ddd}, J=5.7,4.1,2.0 \mathrm{~Hz}), 5.95(1 \mathrm{H}, \mathrm{ddd}, J=5.8,4.2,2.1 \mathrm{~Hz}), 4.60$ (1H, br. s), 4.41-4.35 (1H, m), 2.81 (1H, dddd, $J=17.3,6.3,4.1,2.0 \mathrm{~Hz}), 2.44$ (1H, dm, $J=17.3 \mathrm{~Hz}), 2.02$ (1H, br. s). ${ }^{13} \mathrm{C}$ NMR (100 MHz, $\left.\mathrm{CDCl}_{3}\right): \delta 137.9,134.0,132.1,131.8,130.3,128.8,127.2,126.1,125.6$, 125.4, 123.9, 123.4, 79.6, 56.6, 41.7. IR ( $\mathrm{NaCl}$, neat film): 3347 (br.), 3050, 3010, 2927, 2843, 1595, 1511, 1431, 1396, 1287, 1207, 1066, $1033 \mathrm{~cm}^{-1}$. MS m/z (rel. intensity): 210 ( $\left.\mathrm{M}^{+}, 100\right), 191$ (15), 179 (43), 165 (75), 154 (76), 141 (46), 128 (29), 115 (17). HRMS (ESI) ${ }^{+}$: Calc'd for $\mathrm{C}_{15} \mathrm{H}_{14} \mathrm{O}\left[\mathrm{M}^{+}\right]: 210.104465$; found $=210,105004$. The ee was determined by HPLC analysis : $70 \%$ ee (Chiralcel OD-H, hexane/iPrOH $=98 / 2,0.75 \mathrm{~mL} / \mathrm{min}) ; \mathrm{t}_{\mathrm{R}}=48 \mathrm{~min}(1 \mathrm{~S}, 2 R)$ minor; $\mathrm{t}_{\mathrm{R}}=50.6 \mathrm{~min}(1 R, 2 S)$ major.

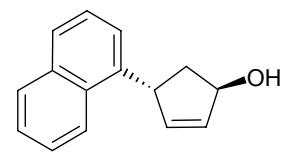

(5o) : (1R, 4R)-trans-4-(1-Naphthalenyl)-cyclopent-2-enol. Colourless oil. ${ }^{1} \mathbf{H}$ NMR $\left(400 \mathrm{MHz}, \mathrm{CDCl}_{3}\right): \delta 8.07(1 \mathrm{H}, \mathrm{d}, J=7.2 \mathrm{~Hz}), 7.88(1 \mathrm{H}, \mathrm{d}, J=7.9 \mathrm{~Hz}), 7.74$ $(1 \mathrm{H}, \mathrm{d}, J=8.2 \mathrm{~Hz}), 7.57(1 \mathrm{H}, \mathrm{dt}, J=7.5,1.5 \mathrm{~Hz}), 7.51(1 \mathrm{H}, \mathrm{dt}, J=8.0,1.4 \mathrm{~Hz}), 7.41$ $(1 \mathrm{H}, \mathrm{t}, J=7.2 \mathrm{~Hz}), 7.34(1 \mathrm{H}, \mathrm{d}, J=6.8 \mathrm{~Hz}), 6.16-6.13(1 \mathrm{H}, \mathrm{m}), 6.05-5.98(1 \mathrm{H}, \mathrm{m})$, 5.15-5.10 $(1 \mathrm{H}, \mathrm{m}), 4.38(1 \mathrm{H}, \mathrm{m}), 3.13(1 \mathrm{H}, \mathrm{ddd}, J=17.4,8.9,2.4 \mathrm{~Hz}), 2.65(1 \mathrm{H}, \mathrm{dddd}, J=17.4,4.0,2.7$, $1.5 \mathrm{~Hz}), 1.82(1 \mathrm{H}, \mathrm{d}, J=6.2 \mathrm{~Hz}) . \mathbf{M S} \mathrm{m} / \mathrm{z}$ (rel. intensity): 210 (M+100), 191 (40), 178 (27), 165 (87), 152 (57), 141 (47), 128 (55), 115 (17), 82 (45). HRMS (ESI) ${ }^{+}$: Calc'd for $\mathrm{C}_{15} \mathrm{H}_{14} \mathrm{O}\left[\mathrm{M}^{+}\right]$: 210.104465; found = 210,105018 . 
(2p) : (1R, 2S)-trans-2-(2-Methylphenyl)-cyclopent-3-enyl ethylcarbonate. Prepared according to the general procedure described above. The crude product was purified by column chromatography (dry loaded). The inseparable regioisomers $\mathbf{2}$ and $\mathbf{3}$ were obtained in $25 \%$ yield $(10 \mathrm{mg})$, showing a $6: 1$ ratio of $2: 3$ by ${ }^{1} \mathrm{H}$ NMR spectroscopy. The regioisomers were submitted directly to the deprotection conditions and characterized as the free alcohols. Major regioisomer 2p: ${ }^{1} \mathbf{H}$ NMR (400 MHz, $\left.\mathrm{CDCl}_{3}\right): \delta 7.19-7.11(3 \mathrm{H}, \mathrm{m}), 7.03-6.98(1 \mathrm{H}, \mathrm{m}), 5.95(1 \mathrm{H}$, ddd, $J=6.0,4.1,2.0 \mathrm{~Hz}), 5.78(1 \mathrm{H}$, ddd, $J=6.9,4.3,2.0 \mathrm{~Hz}), 5.06(1 \mathrm{H}, \mathrm{dt}, J=6.5,2.6 \mathrm{~Hz}), 4.24-4.21$ $(1 \mathrm{H}, \mathrm{m}), 4.18(2 \mathrm{H}, \mathrm{q}, J=7.1 \mathrm{~Hz}), 2.94(1 \mathrm{H}, \mathrm{dddd}, J=17.8,6.4,4.1,2.2 \mathrm{~Hz}), 2.47(1 \mathrm{H}, \mathrm{dddd}, J=17.8,4.3$, 2.6, $1.0 \mathrm{~Hz}), 2.40(3 \mathrm{H}, \mathrm{s}), 1.31(3 \mathrm{H}, \mathrm{t}, J=7.1 \mathrm{~Hz})$.

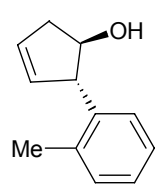

(4p) : (1R, 2S)-trans-2-(2-Methylphenyl)-cyclopent-3-enol. Obtained by methanolysis of the isolated carbonates mixture (vide supra) and purified by column chromatography (10-25\% EtOAc/hexanes gradient). The alcohol was quantitavely isolated as a colourless oil. The characterization data was fully concordant with that already reported. ${ }^{10 \mathrm{~b}}{ }^{1} \mathbf{H}$ NMR $(400 \mathrm{MHz}$, $\left.\mathrm{CDCl}_{3}\right): \delta$ 7.22-7.09 $(3 \mathrm{H}, \mathrm{m}), 7.03-6.98(1 \mathrm{H}, \mathrm{m}), 5.96(1 \mathrm{H}, \mathrm{ddd}, J=6.1,4.2,2.0 \mathrm{~Hz}), 5.77(1 \mathrm{H}, \mathrm{ddd}, J=$ 6.9, 4.1, $2.1 \mathrm{~Hz}), 4.29-4.22(1 \mathrm{H}, \mathrm{m}), 4.04(1 \mathrm{H}, \mathrm{br} . \mathrm{s}), 2.79(1 \mathrm{H}, \mathrm{dddd}, J=17.8,6.1,4.2,2.0 \mathrm{~Hz}), 2.47(3 \mathrm{H}$, s), $2.34(1 \mathrm{H}$, br. d, $J=17.7 \mathrm{~Hz}), 2.40(3 \mathrm{H}, \mathrm{s}), 1.79\left(1 \mathrm{H}\right.$, br. s). MS $\mathrm{m} / \mathrm{z}$ (rel. intensity): $174\left(\mathrm{M}^{+}, 100\right), 156$ (22), 131 (52), 128 (39), 118 (72), 115 (37), 105 (41), 91 (33). HRMS (ESI) ${ }^{+}$: Calc'd for $\mathrm{C}_{12} \mathrm{H}_{14} \mathrm{O}$ [M $\mathrm{M}^{+}$: 174.104465; found $=174.104454$. The ee was determined by HPLC analysis : $92 \%$ ee (Chiralcel OD-H, hexane $/ \mathrm{iPrOH}=98 / 2,1.0 \mathrm{~mL} / \mathrm{min}) ; \mathrm{t}_{\mathrm{R}}=15.4 \mathrm{~min}(1 S, 2 R)$ minor; $\mathrm{t}_{\mathrm{R}}=20.4 \mathrm{~min}(1 R, 2 S)$ major.

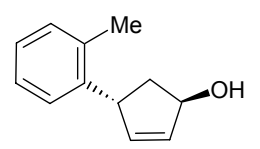

(5p) : (1R, 4R)-trans-4-(2-Methylphenyl)-cyclopent-2-enol. Colourless oil. The characterization data was fully concordant with that already reported. ${ }^{10 b}{ }^{1} \mathbf{H}$ NMR (400 $\left.\mathrm{MHz}, \mathrm{CDCl}_{3}\right): \delta 7.16-7.10(3 \mathrm{H}, \mathrm{m}), 7.00(1 \mathrm{H}, \mathrm{dd}, J=6.3,1.7 \mathrm{~Hz}), 6.09-6.05(2 \mathrm{H}, \mathrm{m})$, $5.03(1 \mathrm{H}$, br. s), $4.36(1 \mathrm{H}, \mathrm{dt}, J=5.7,1.7 \mathrm{~Hz}), 2.36(3 \mathrm{H}, \mathrm{s}), 2.32(1 \mathrm{H}, \mathrm{ddd}, J=14.2,8.2$, $2.6 \mathrm{~Hz}), 1.98(1 \mathrm{H}, \mathrm{ddd}, J=14.1,6.8,5.8 \mathrm{~Hz}), 0.88(1 \mathrm{H}$, br. s). 
(2a) : (1R, 2S)-trans-2-phenylcyclopent-3-enyl-ethylcarbonate

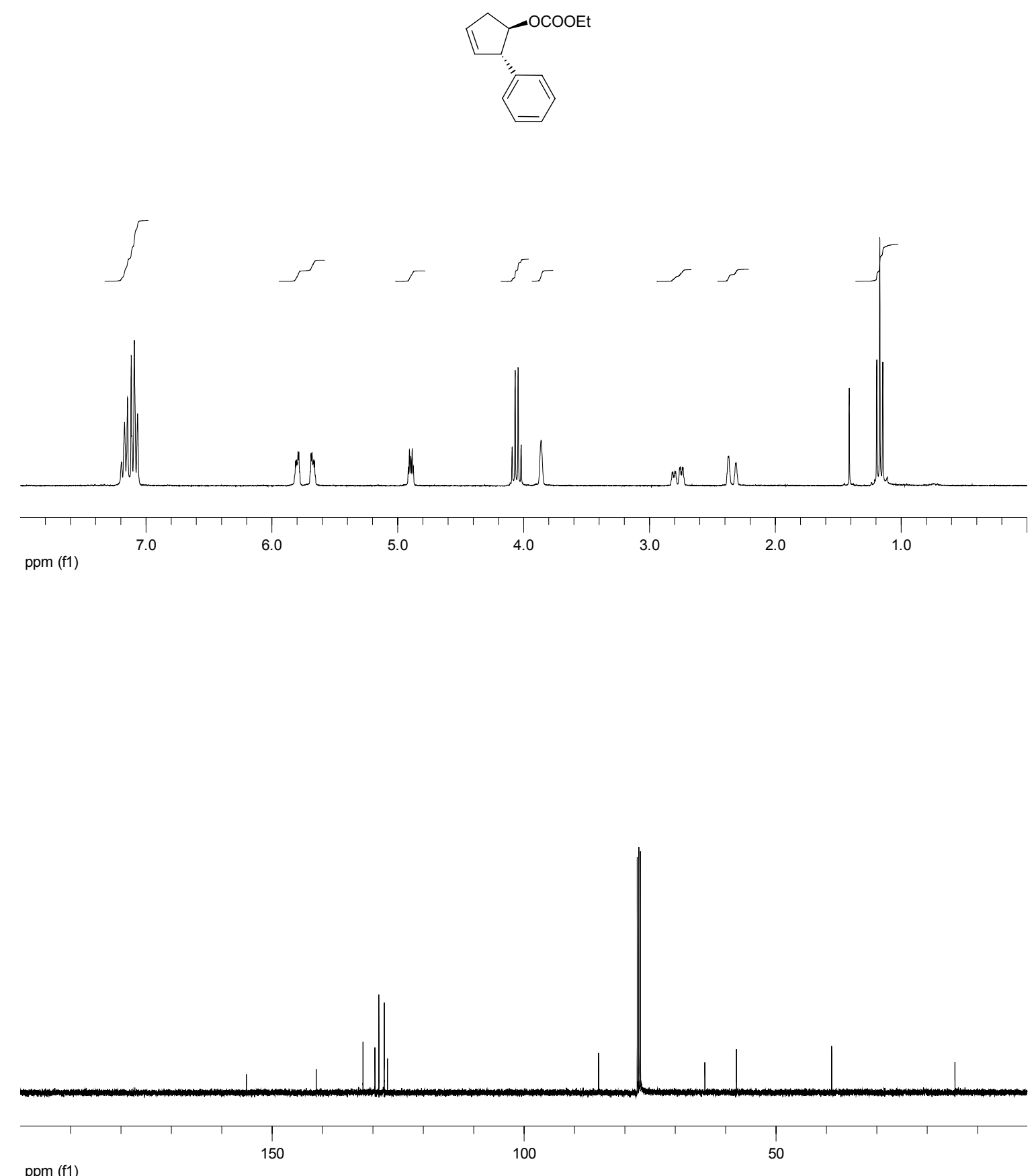

ppm (f1) 
(4a) : (1R, 2S)-trans-2-phenylcyclopent-3-enol

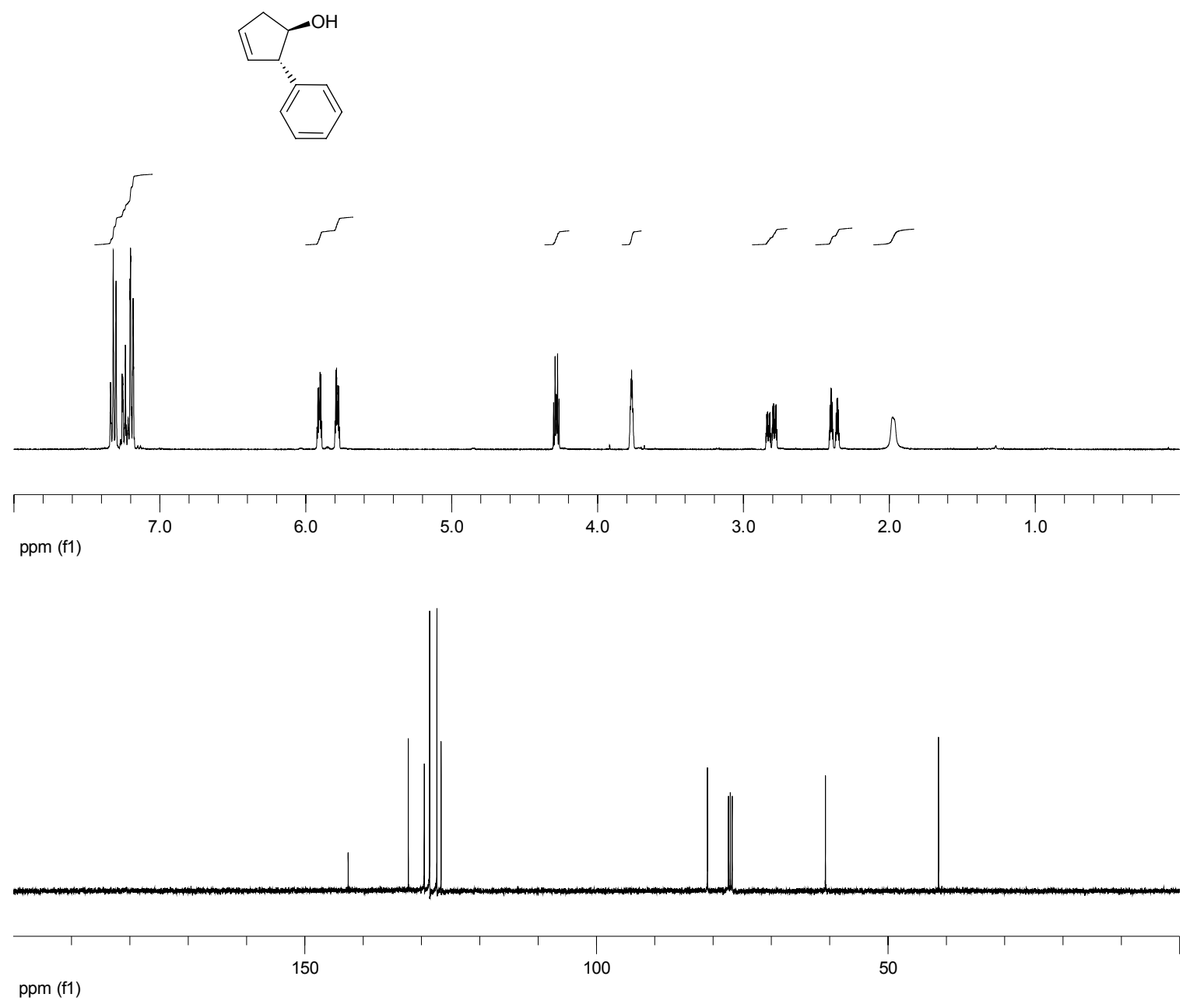

(5a) : (1R, 4R)-trans-4-phenylcyclopent-2-enol

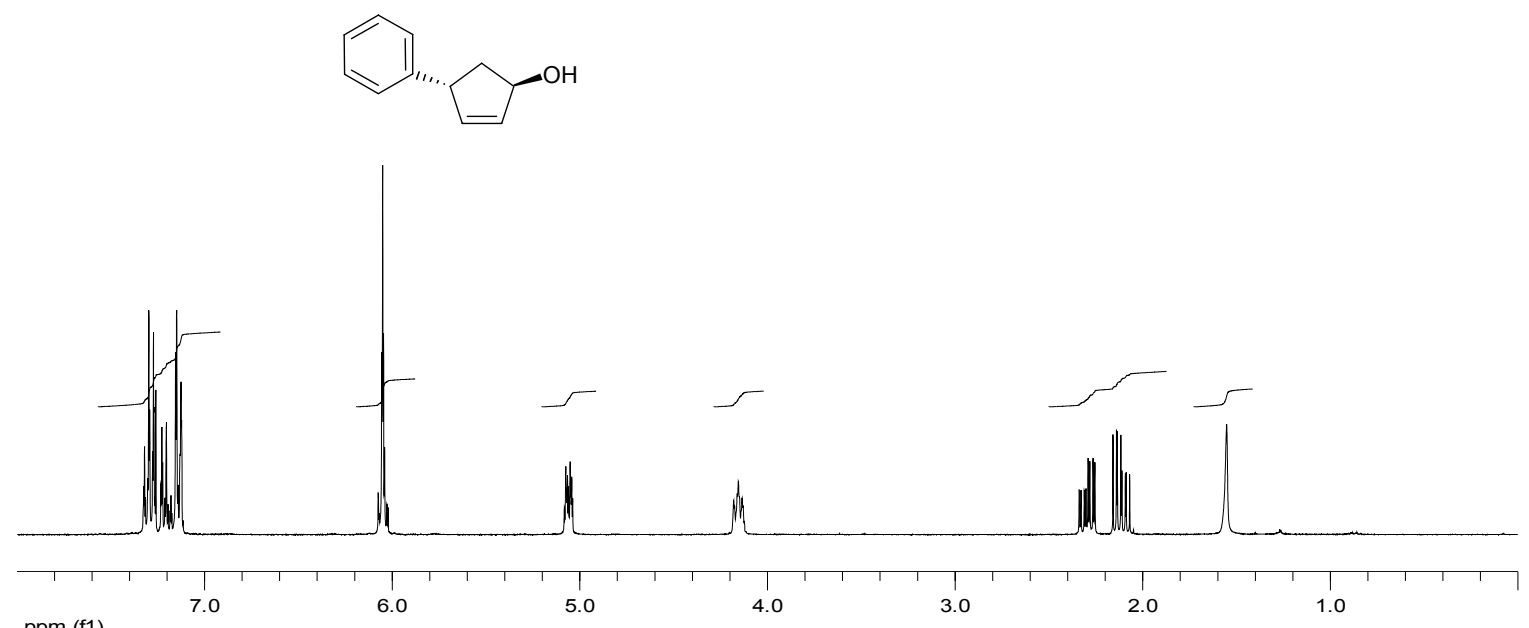

ppm (f1) 
(4b) : (1R, 2S)-trans-2-(4-Methoxycarbonylphenyl)-cyclopent-3-enol.
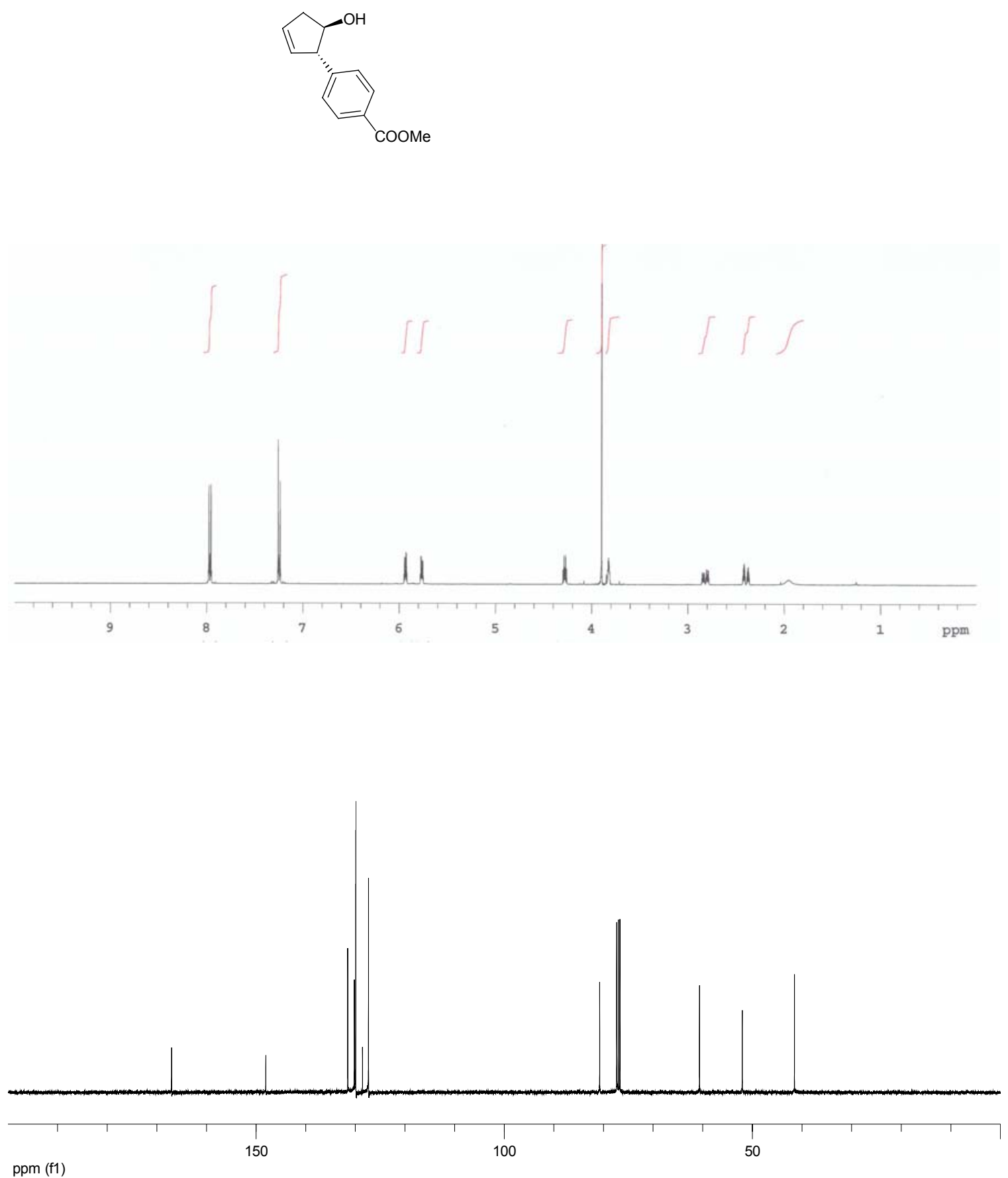

ppm (f1) 
(4c) : (1R, 2S)-trans-2-(4-Acetylphenyl)-cyclopent-3-enol.
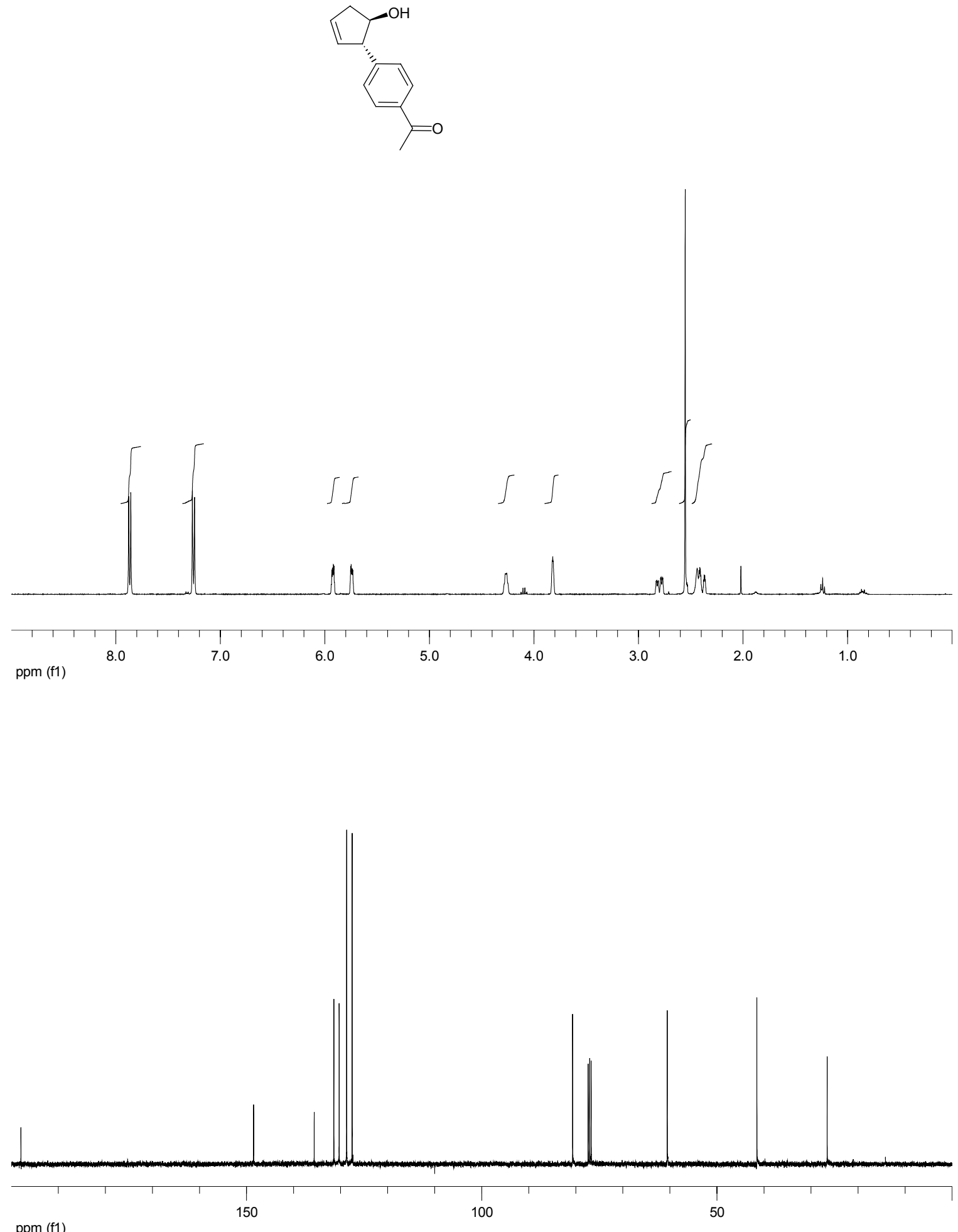

ppm (f1) 
(4d) : (1R, 2S)-trans-2-(4'-Trifluoromethylphenyl)-cyclopent-2-enol.
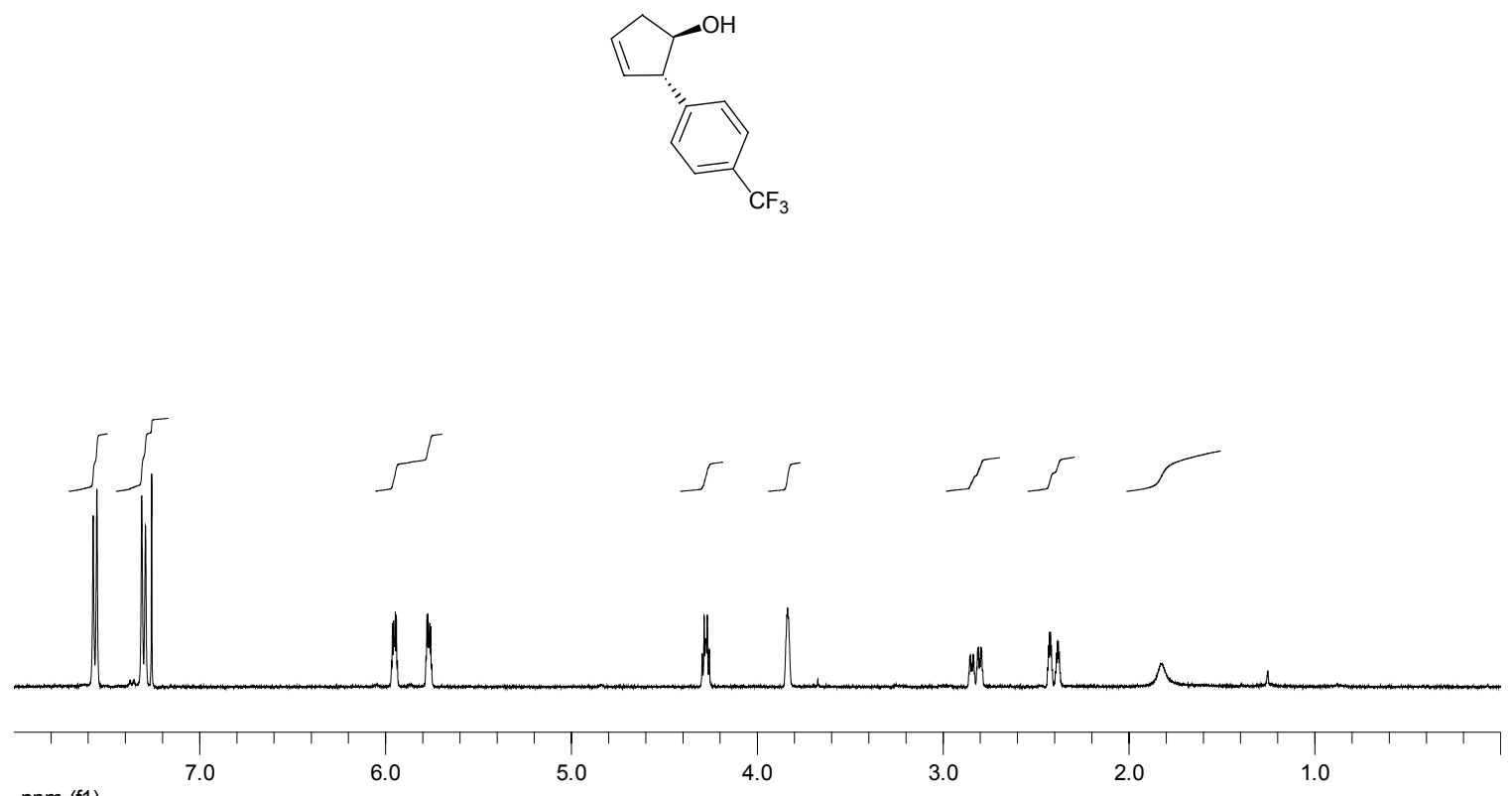

ppm (f1)

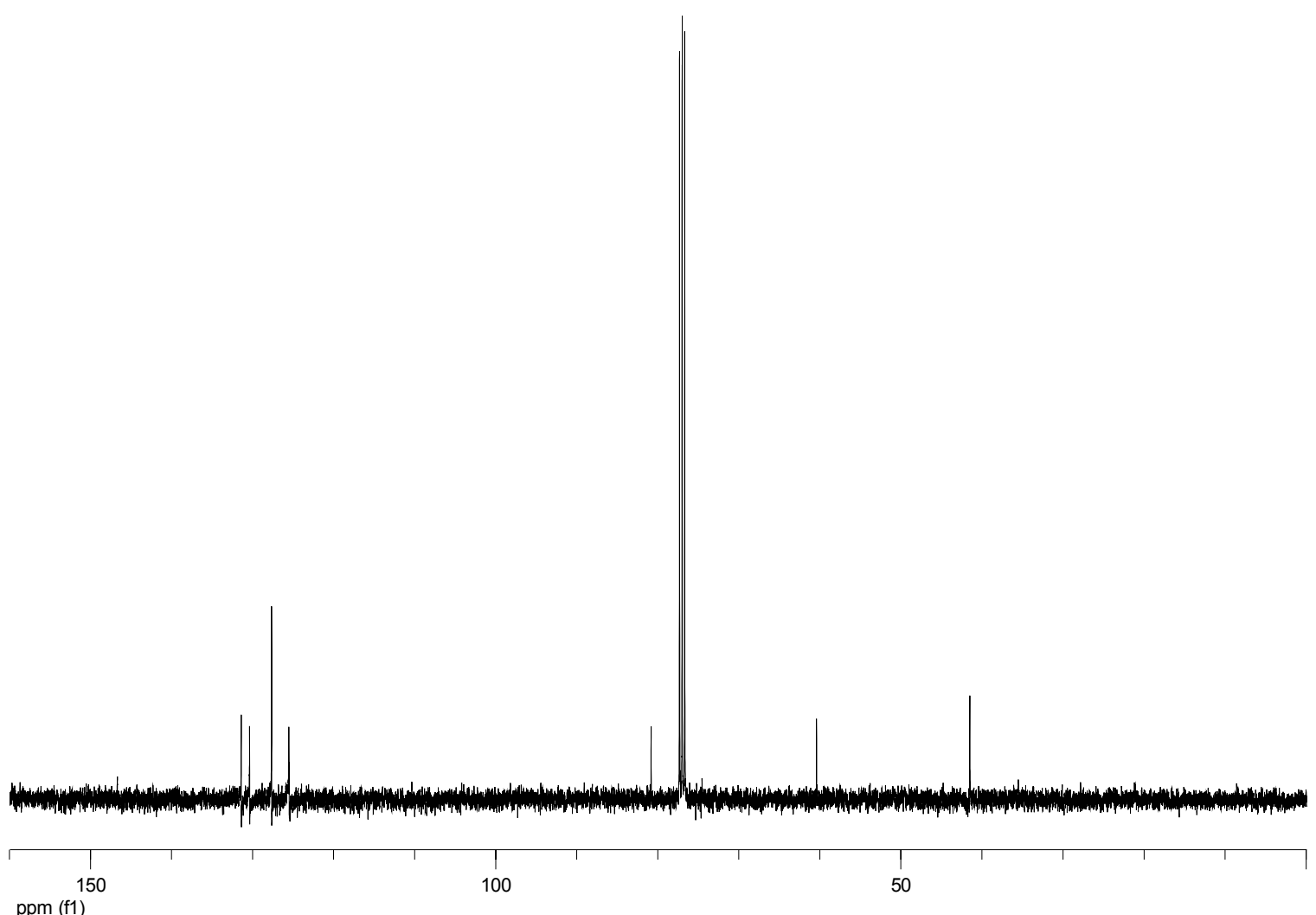


(4e) : (1R, 2S)-trans-2-(4-Fluorophenyl)-cyclopent-3-enol.
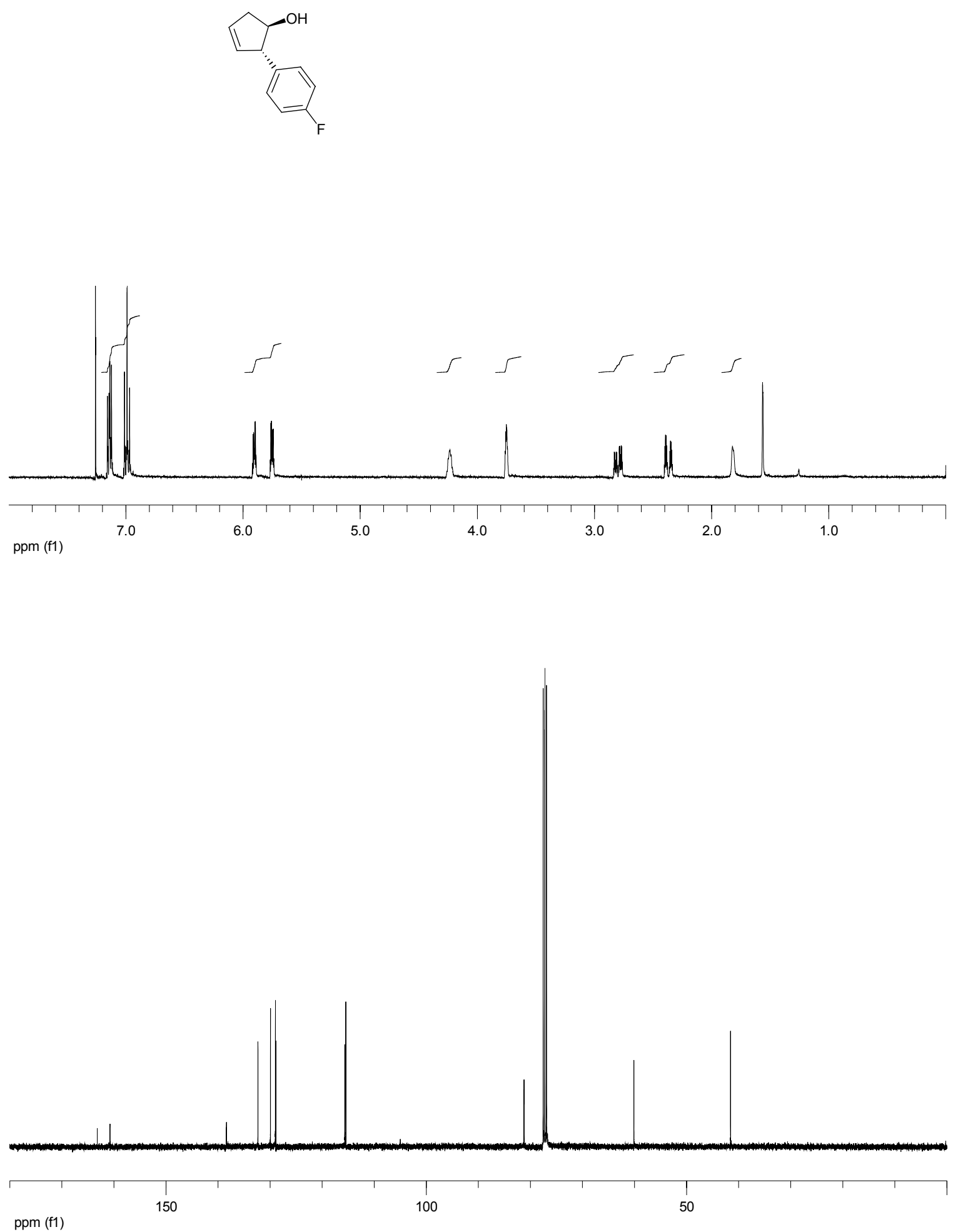
(4f) : (1R, 2S)-trans-2-(4-Chlorophenyl)-cyclopent-3-enol.
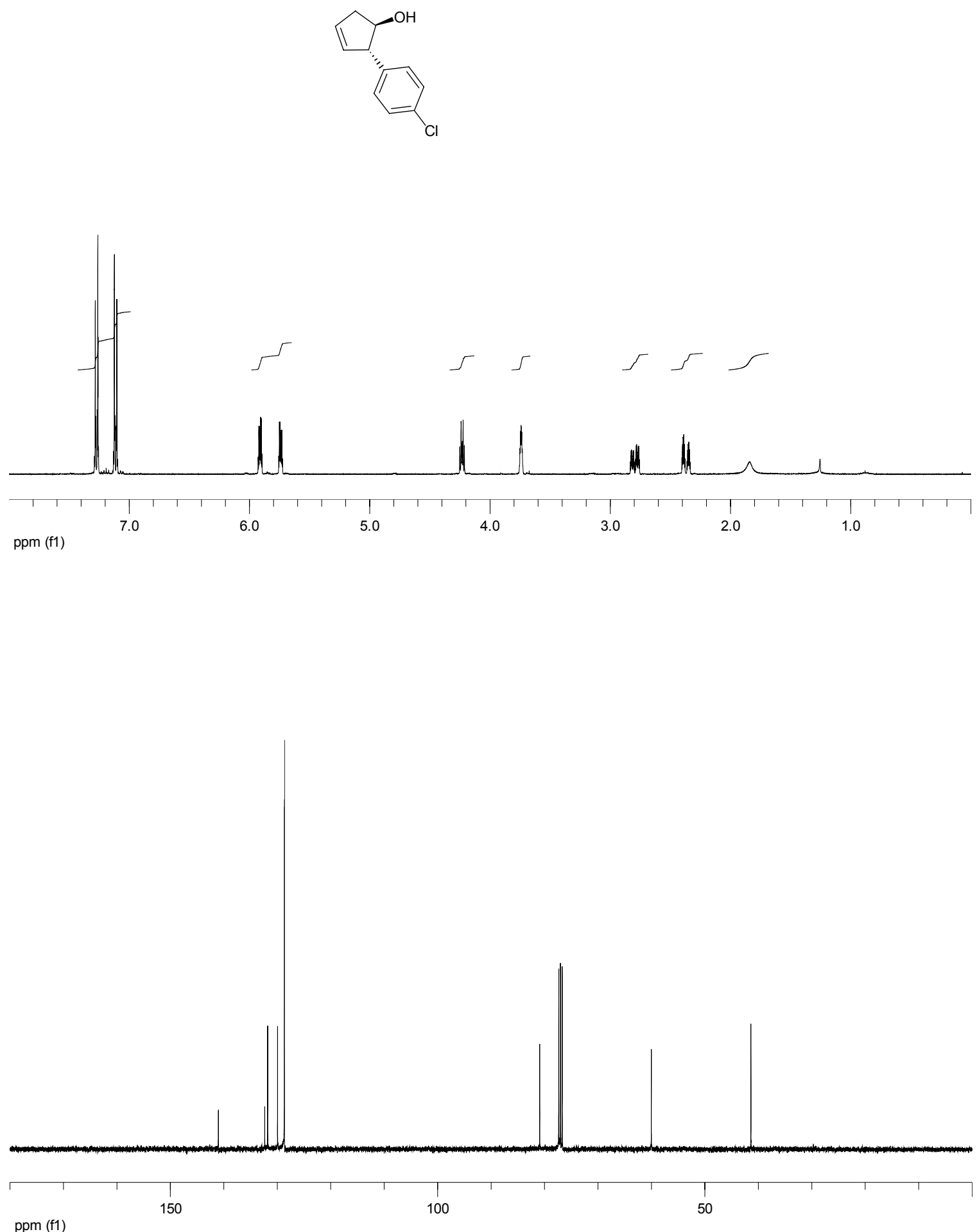
(4g) : (1R, 2S)-trans-2-(4-Bromophenyl)-cyclopent-3-enol.
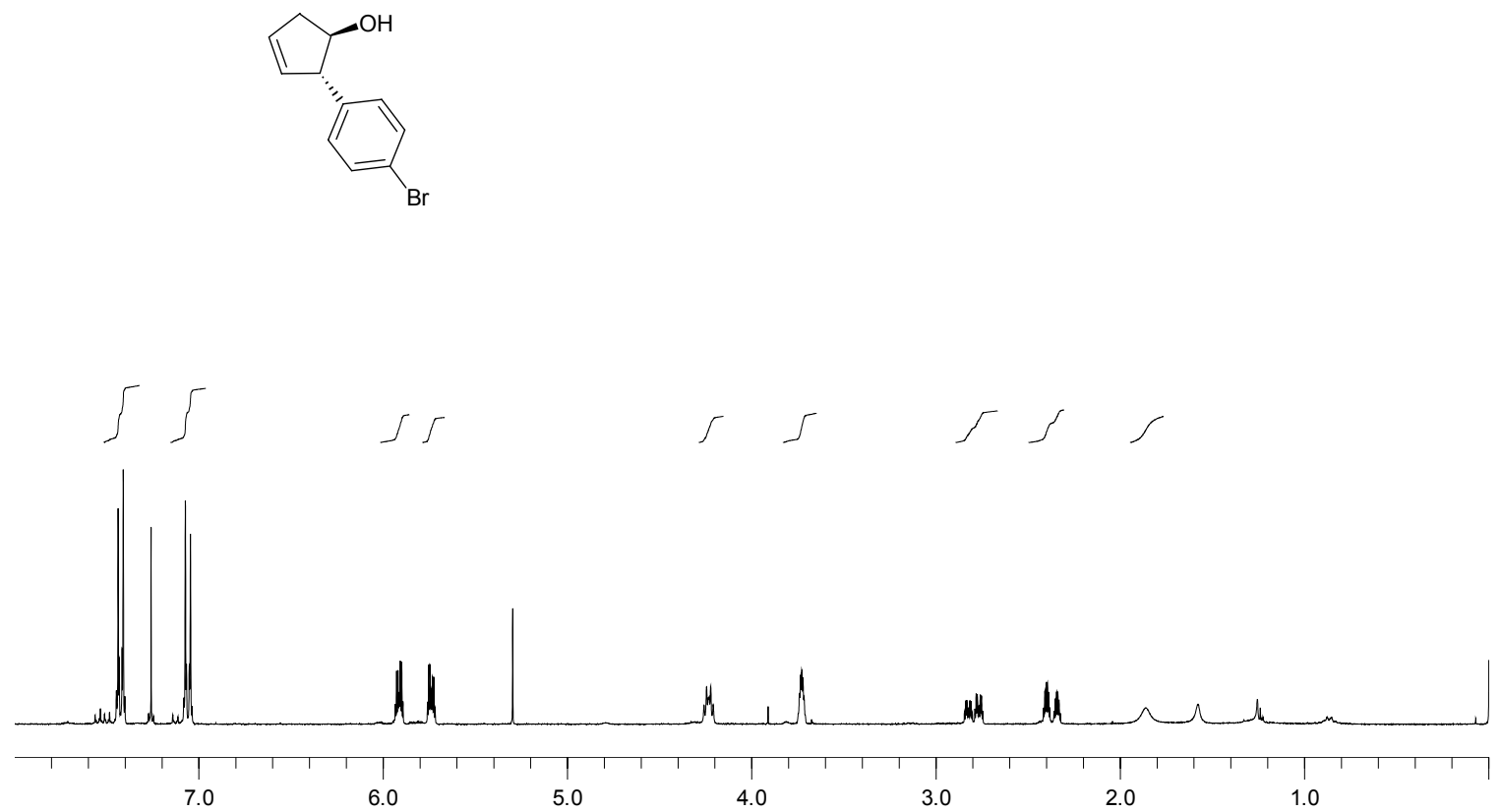

ppm (f1)

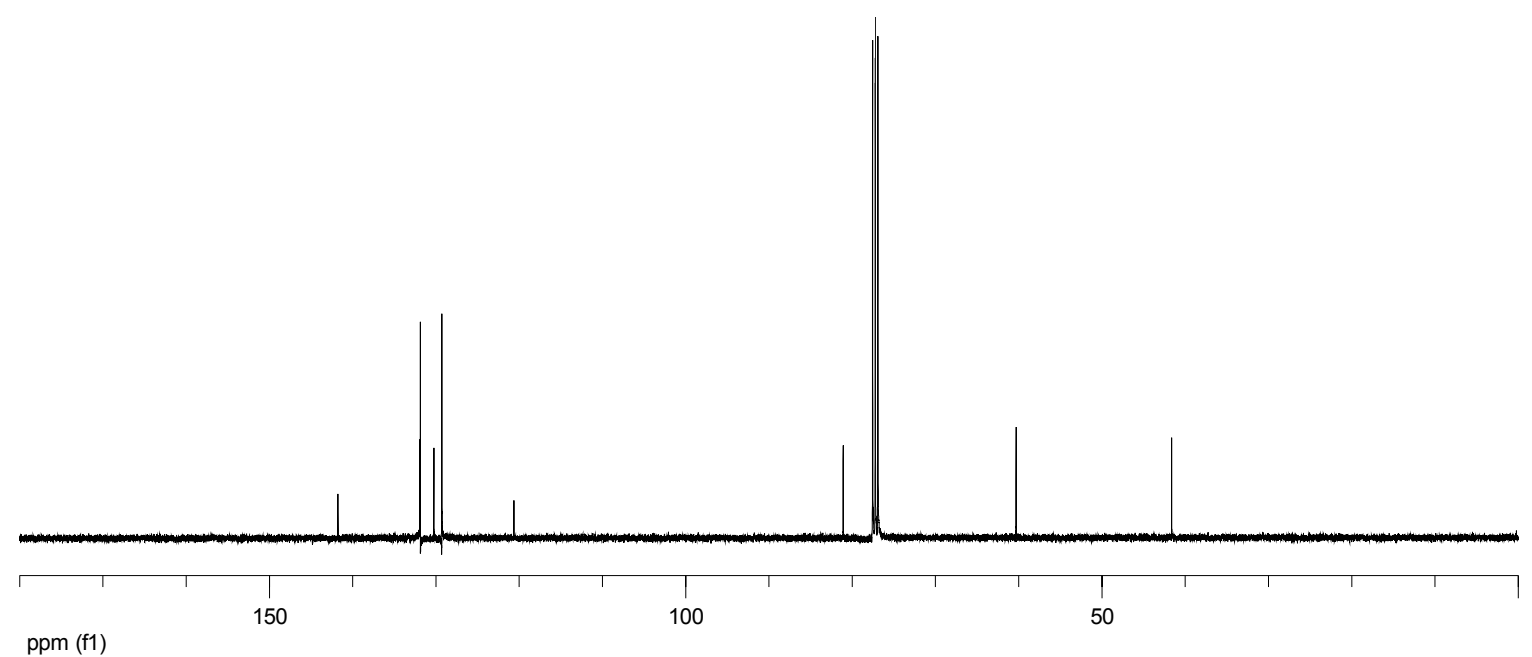


(4h) : (1R, 2S)-trans-2-(4-Methylphenyl)-cyclopent-3-enol.

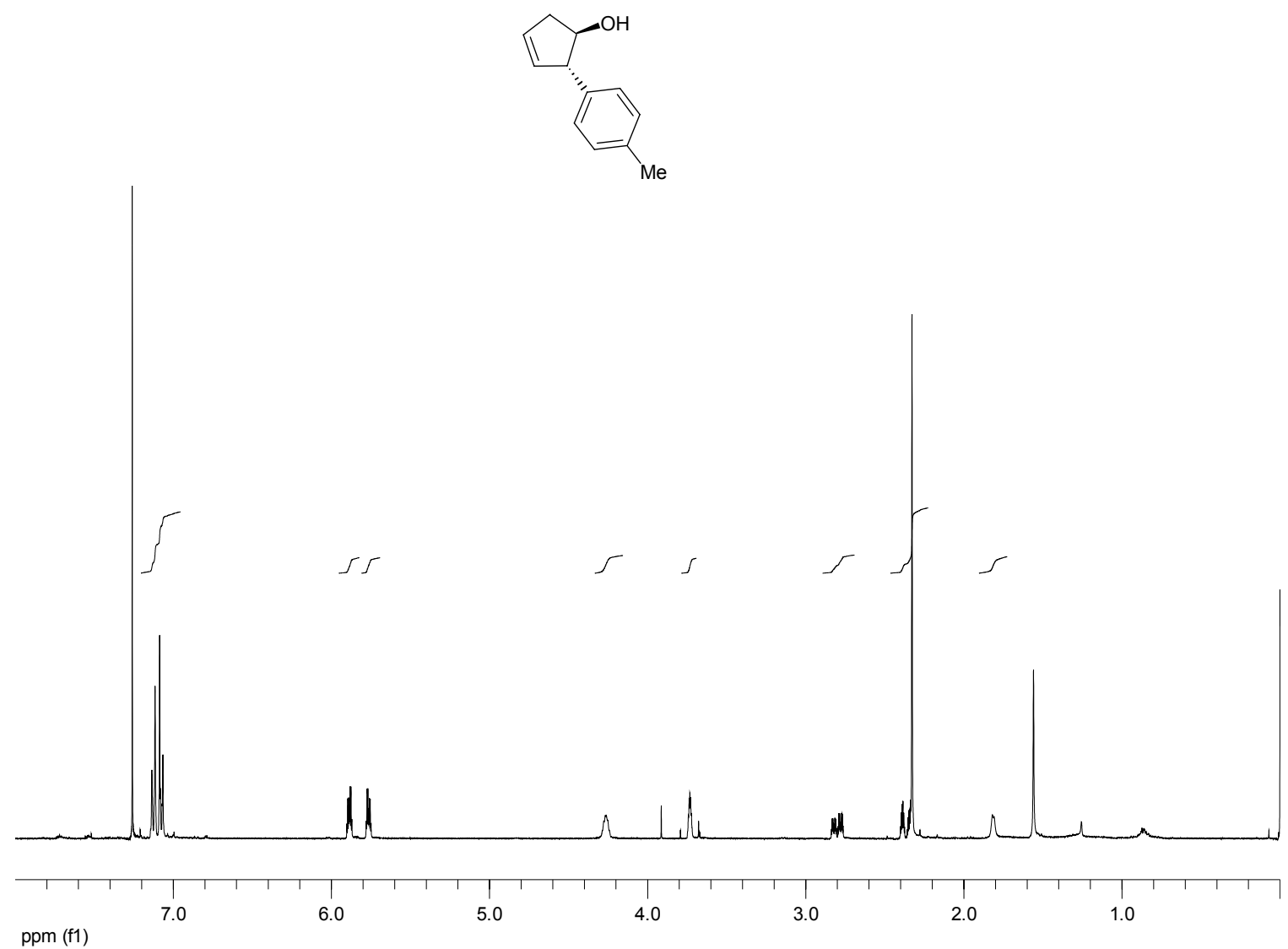

ppm (f1)

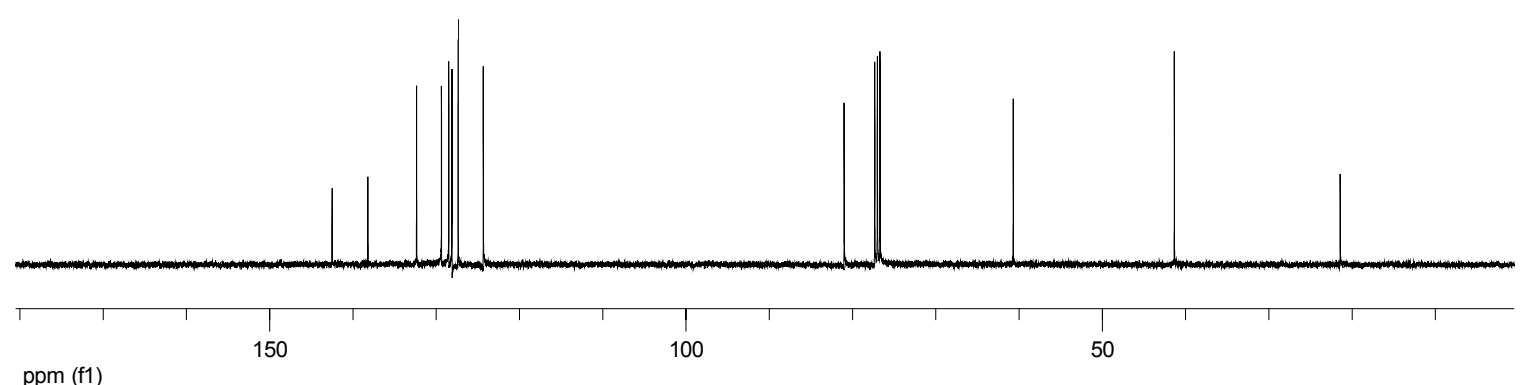

ppm (f1) 
Menard, F.; Chapman, T.; Dockendorff, C. J.; Lautens, M. 
(4i) : (1R, 2S)-trans-2-(4-[t-Butoxycarbonylamino]-phenyl)-cyclopent-3-enol.
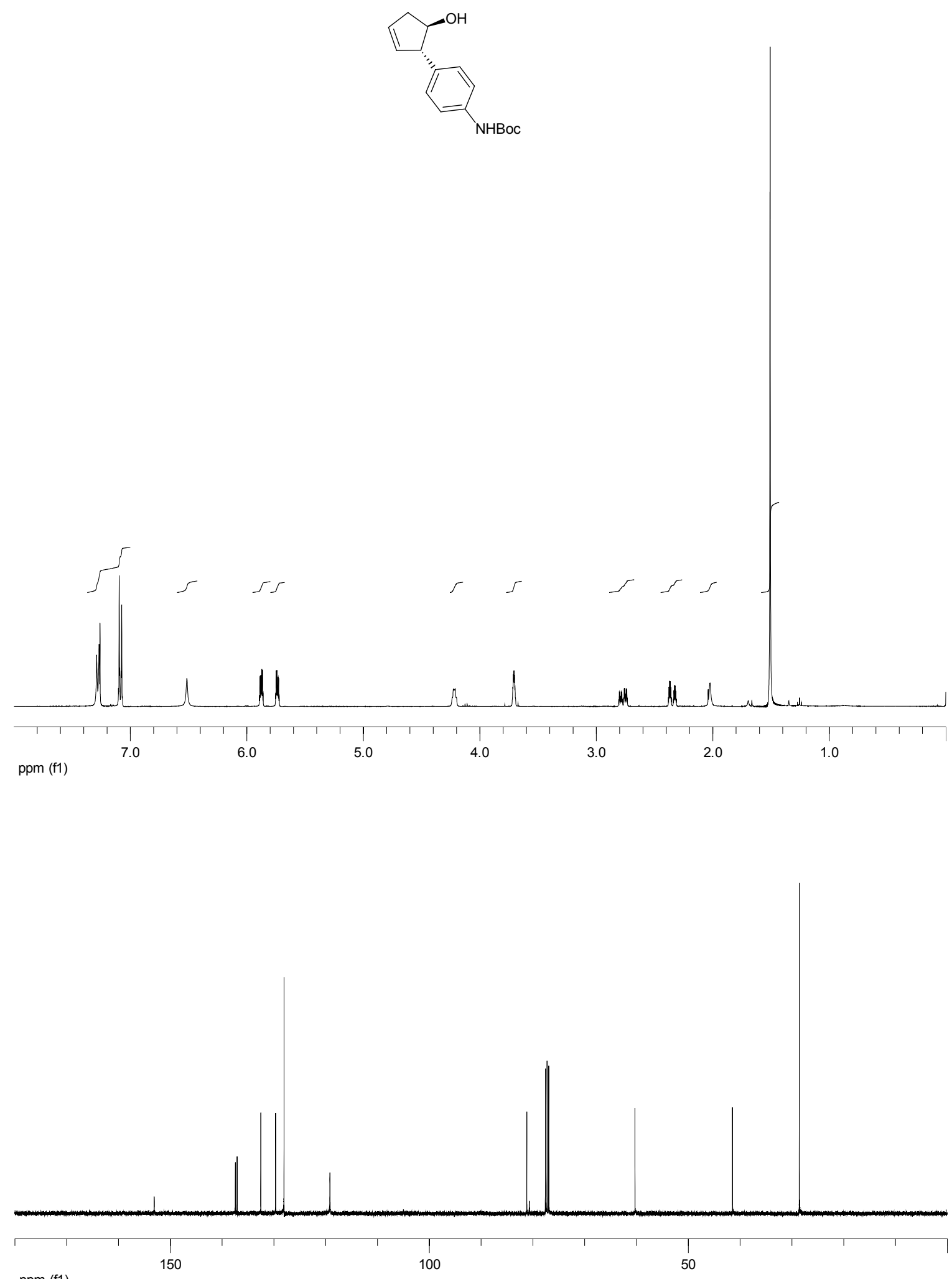

ppm (f1) 
(4j) : (1R, 2S)-trans-2-(4-Methoxyphenyl)-cyclopent-3-enol.

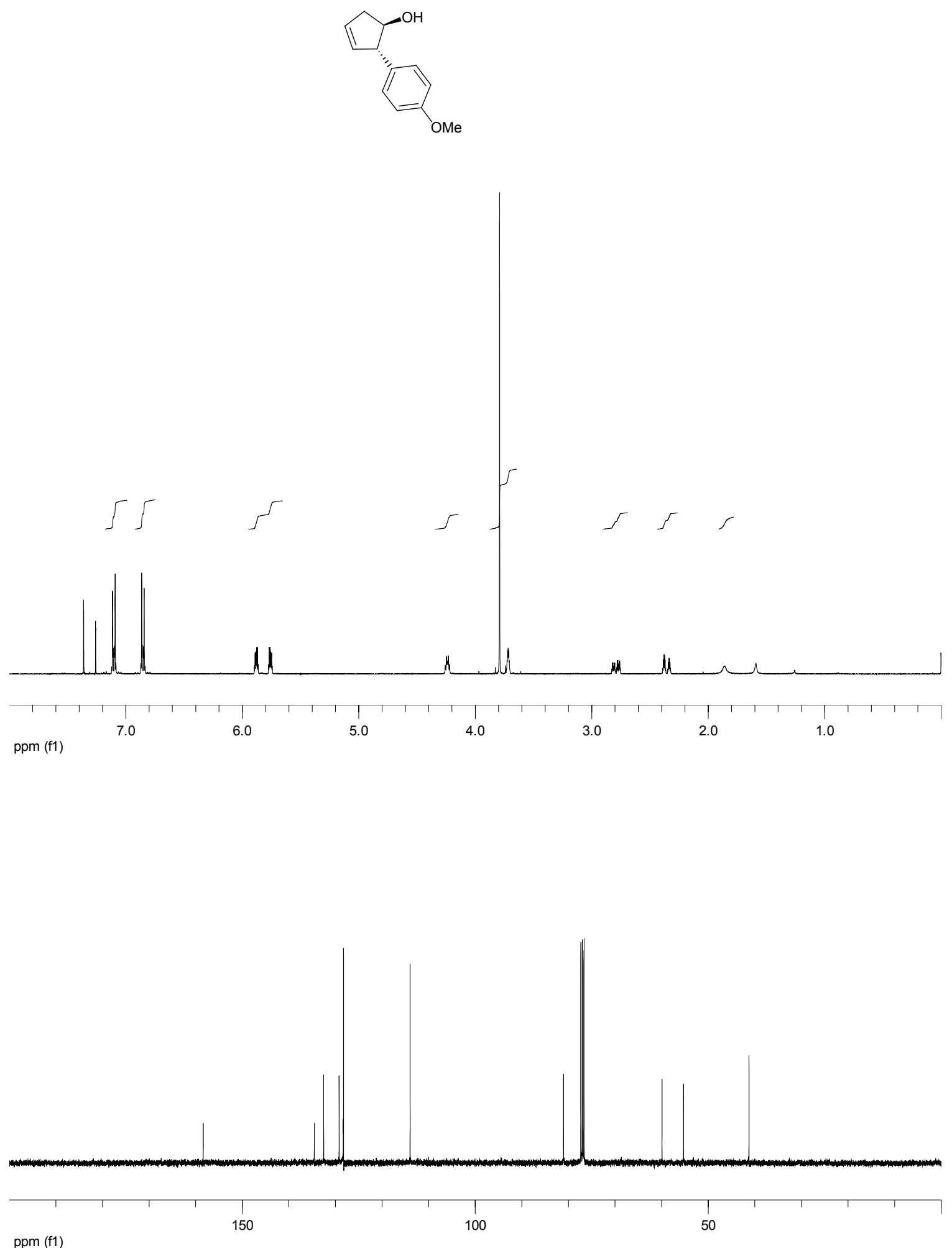

ppm (f1) 
(4k) : (1R, 2S)-trans-2-(3-Methoxyphenyl)-cyclopent-3-enol.

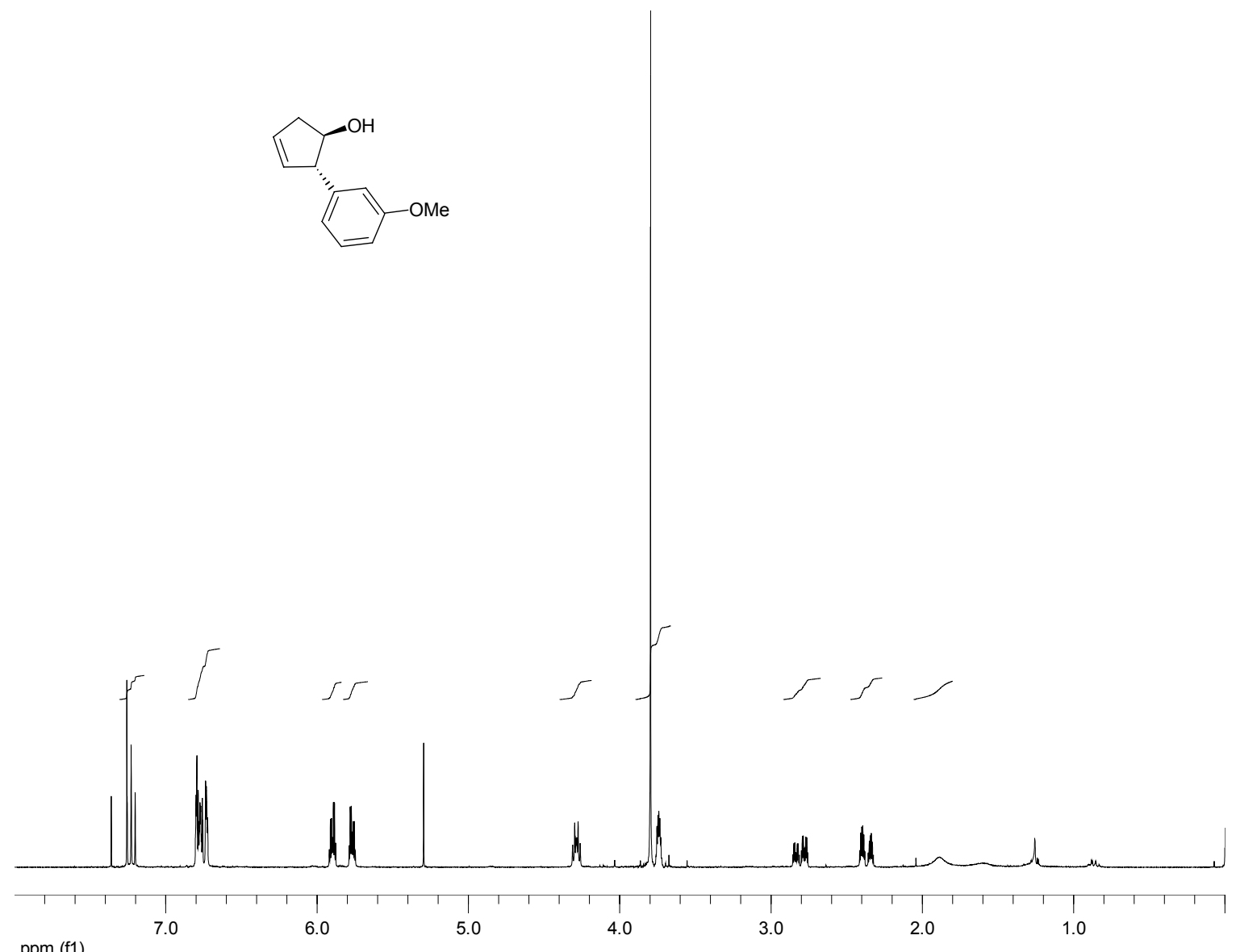

ppm (f1)

$7.0-2.0$

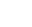

(1)


(4l) : (1R, 2S)-trans-2-(3-Chlorophenyl)-cyclopent-3-enol.
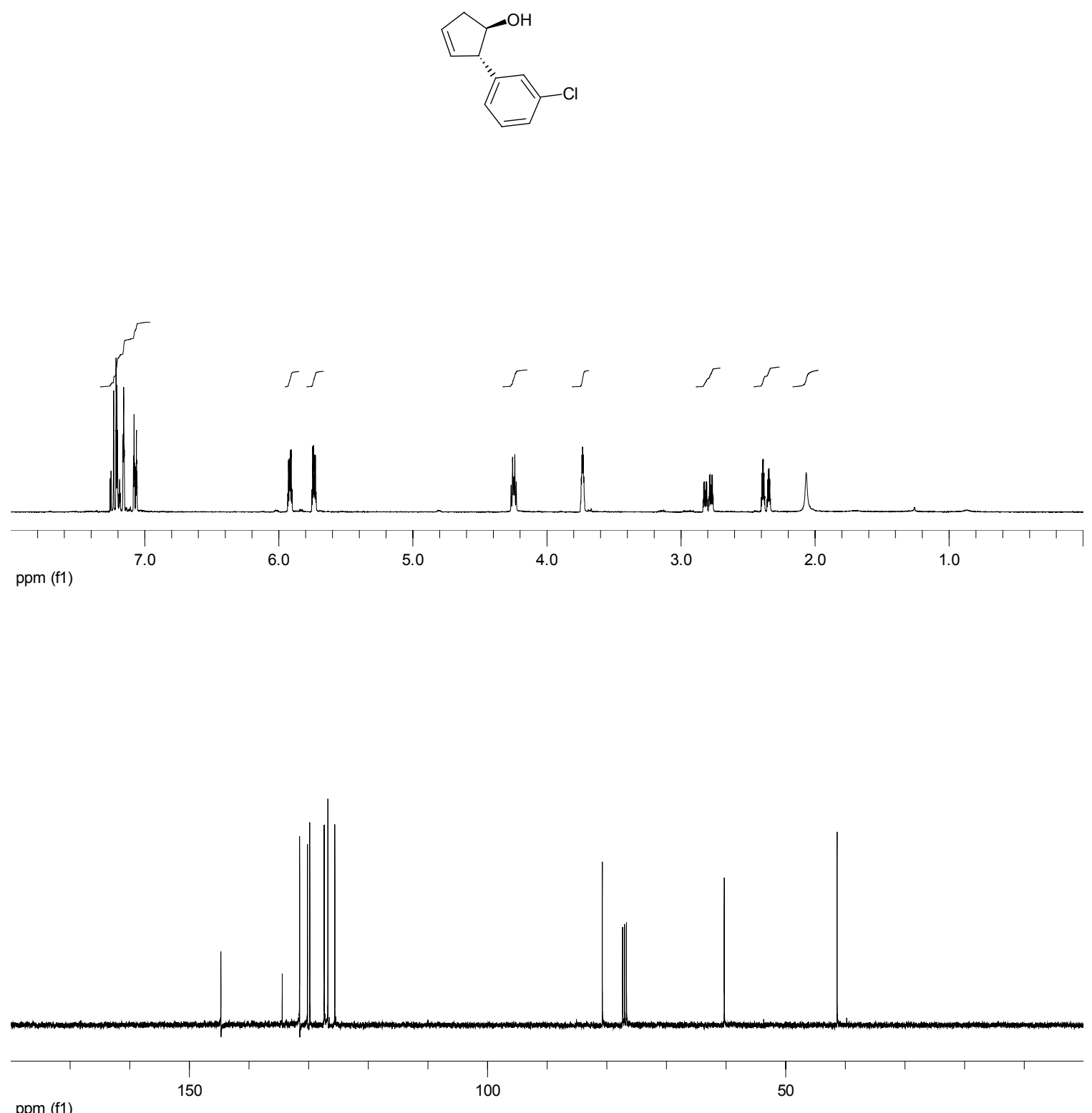
(4m) : (1R, 2S)-trans-2-(3-Methylphenyl)-cyclopent-3-enol.
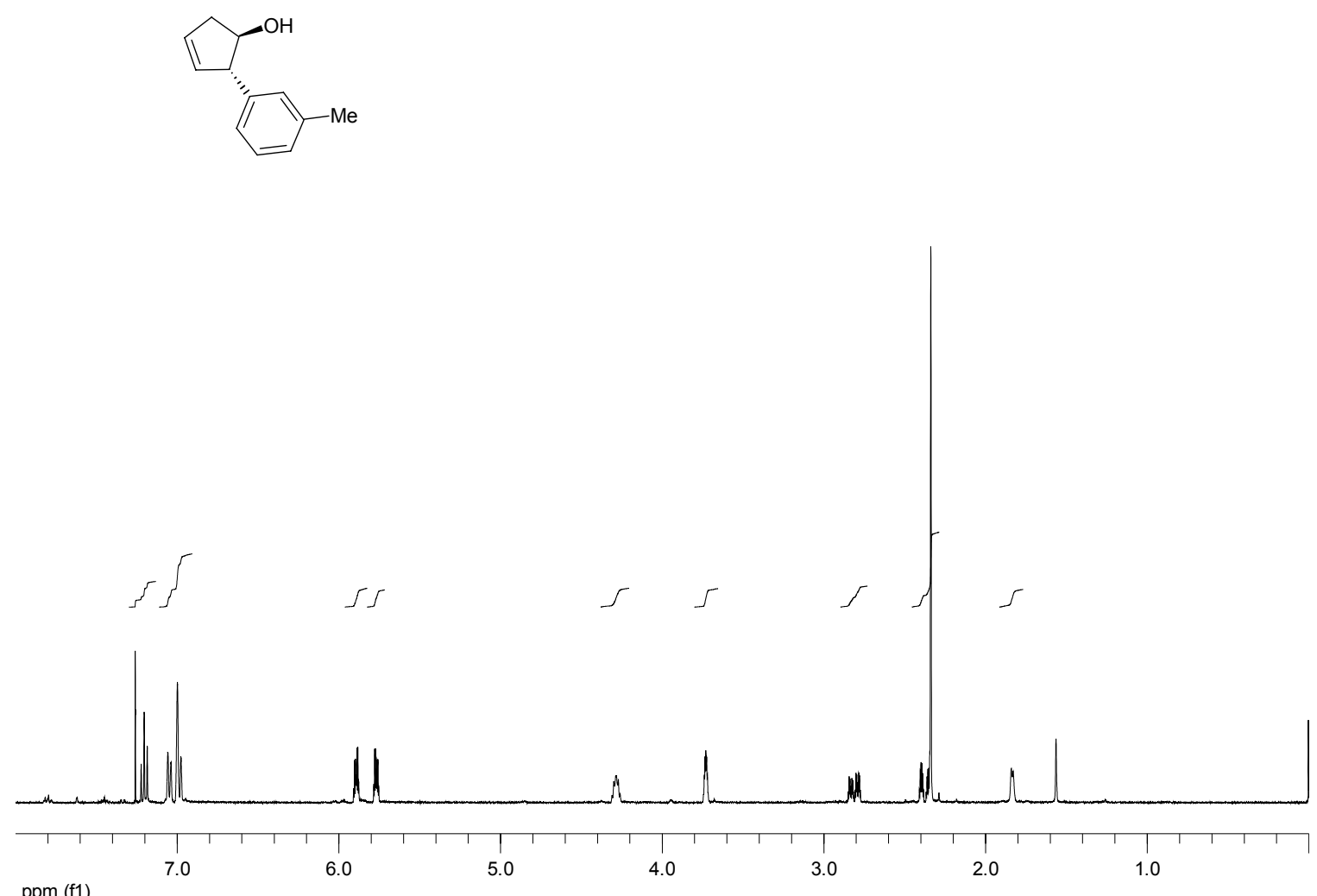

ppm (f1)
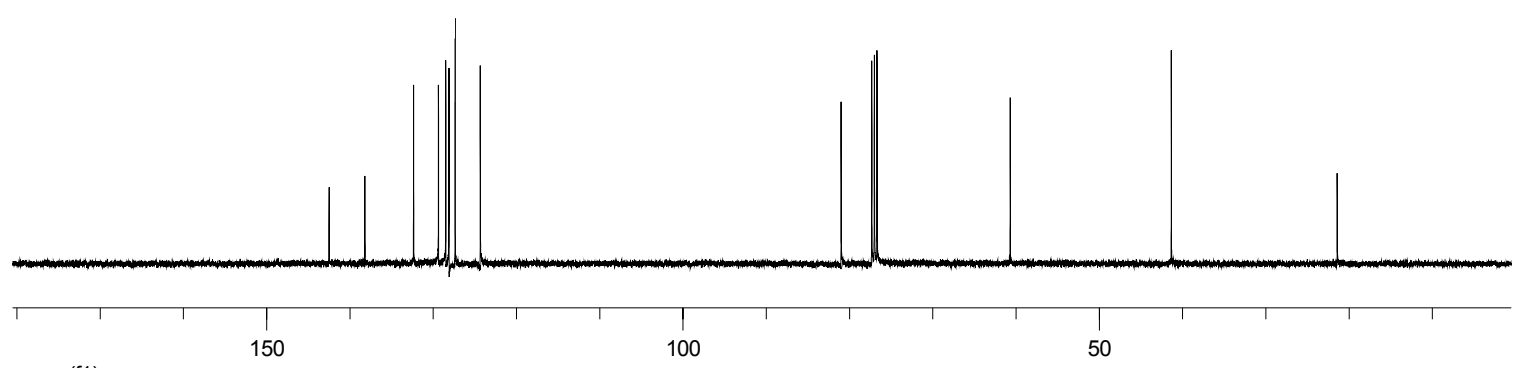

ppm (f1) 
(4n) : (1R, 2S)-trans-2-(2-Naphthalenyl)-cyclopent-3-enol.
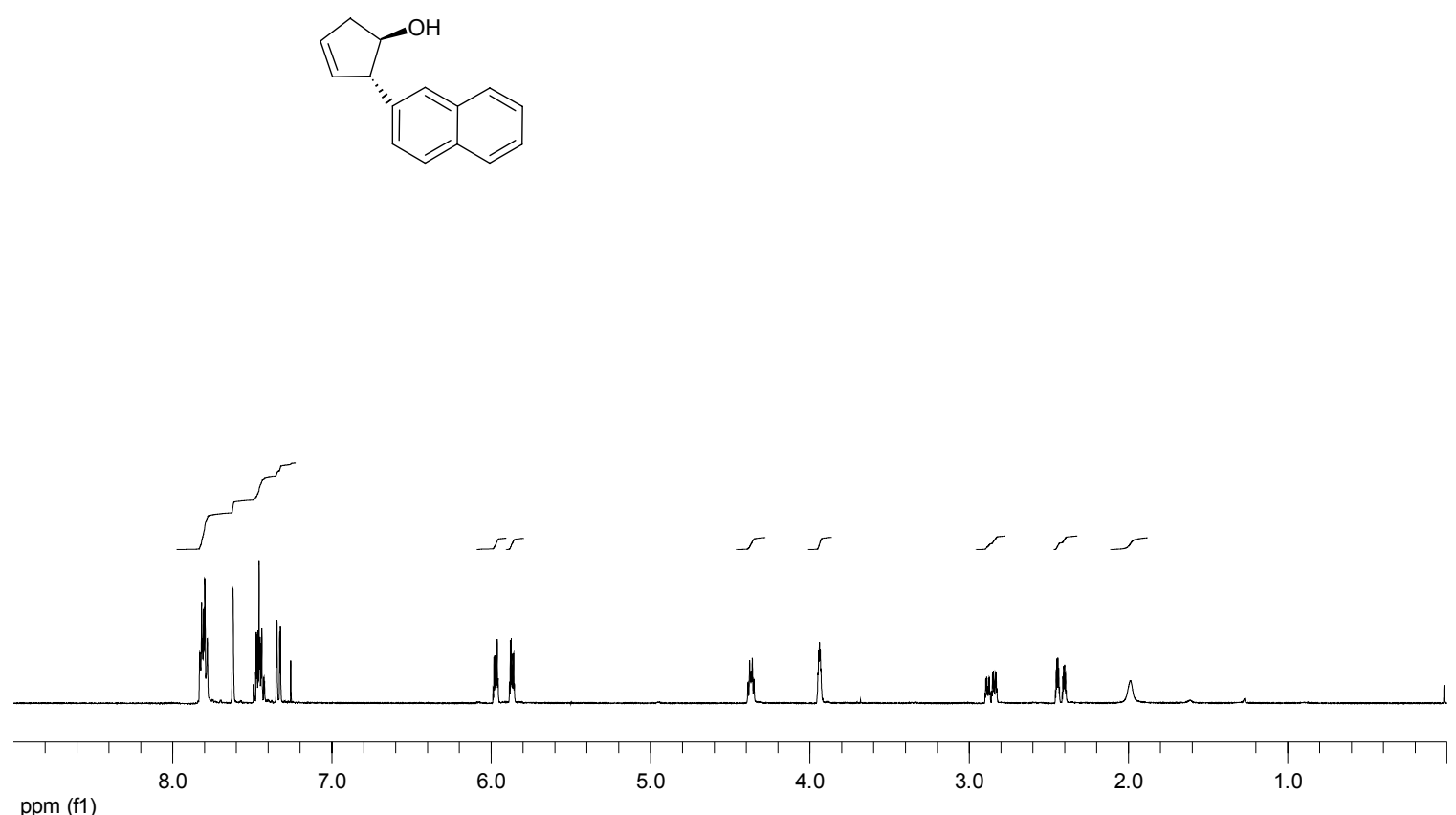

ppm (f1)

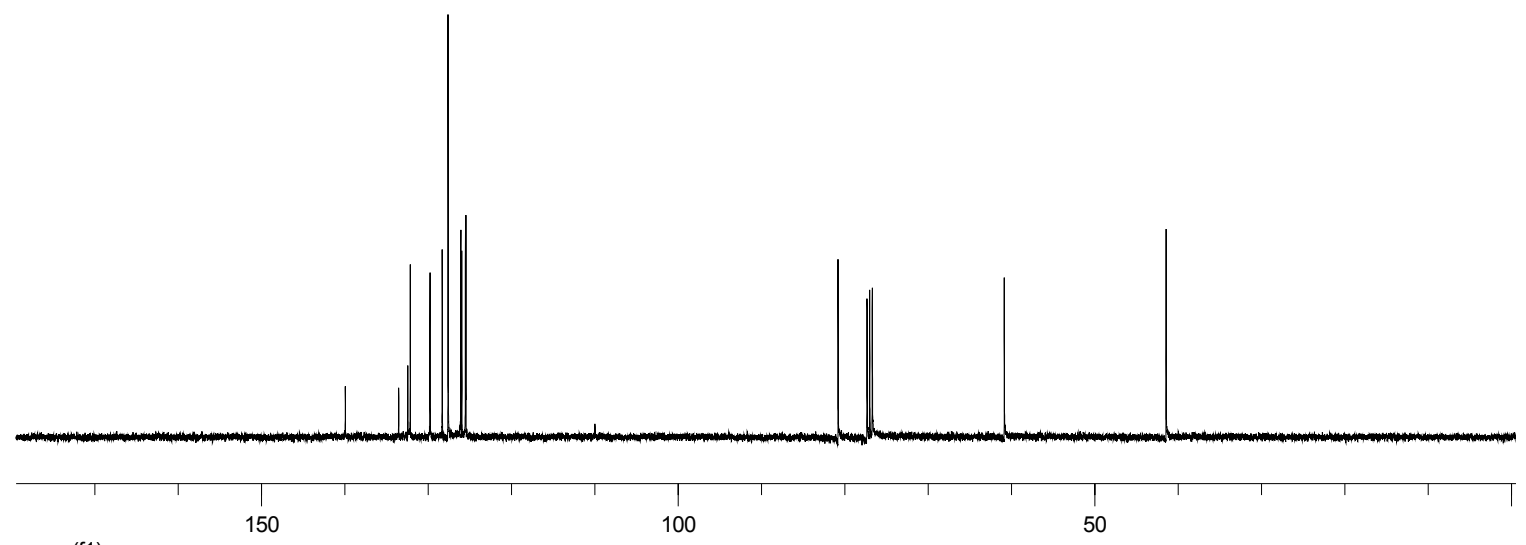

ppm (f1) 
(40) : (1R, 2S)-trans-2-(1-Naphthalenyl)-cyclopent-3-enol.

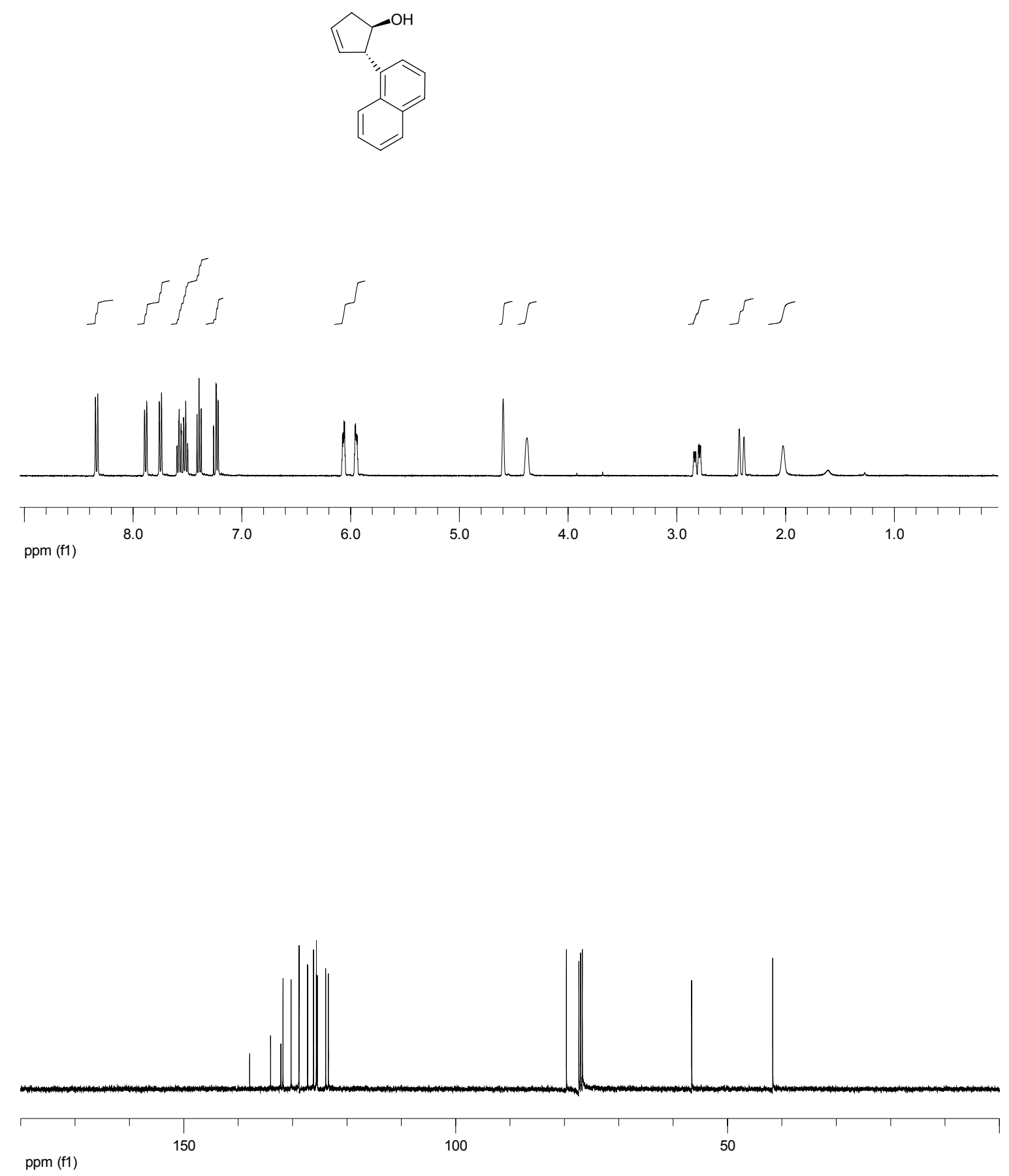


(4p) : (1R, 2S)-trans-2-(2-Methylphenyl)-cyclopent-3-enol.
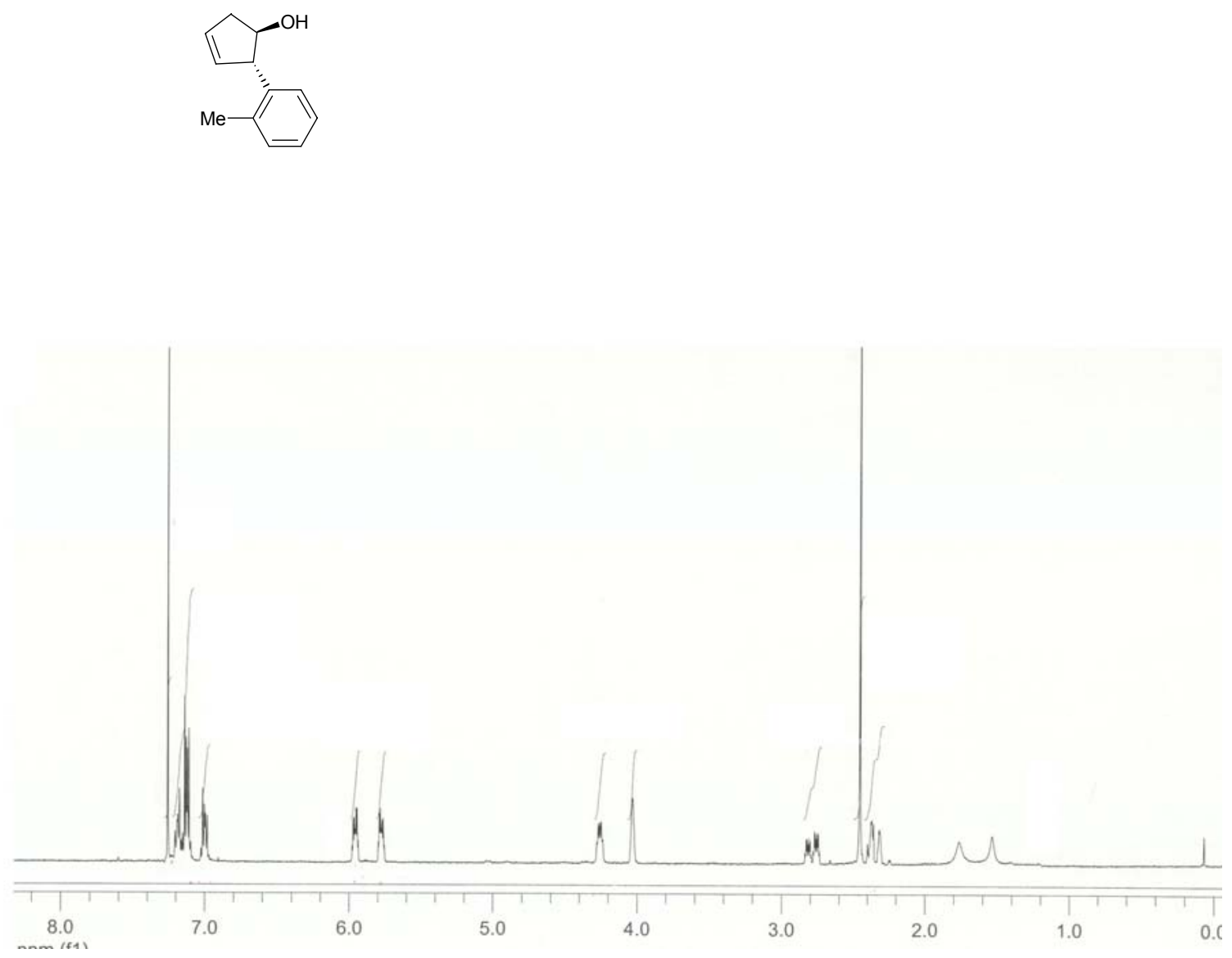
(2b) : (1R, 2S)-trans-2-(4-Methoxycarbonyl-phenyl)-cyclopent-3-enyl ethyl-carbonate.

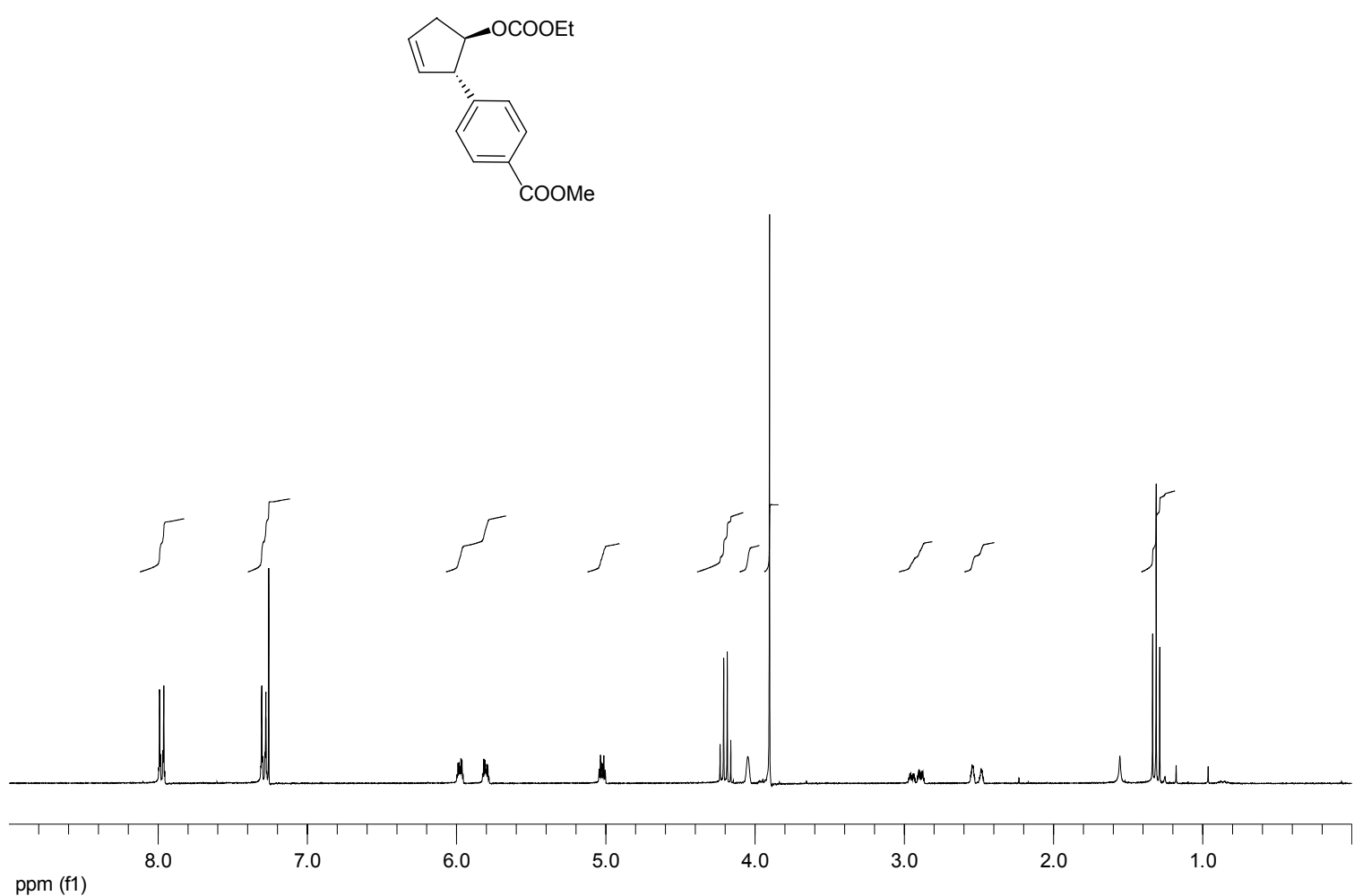

ppm (f1)

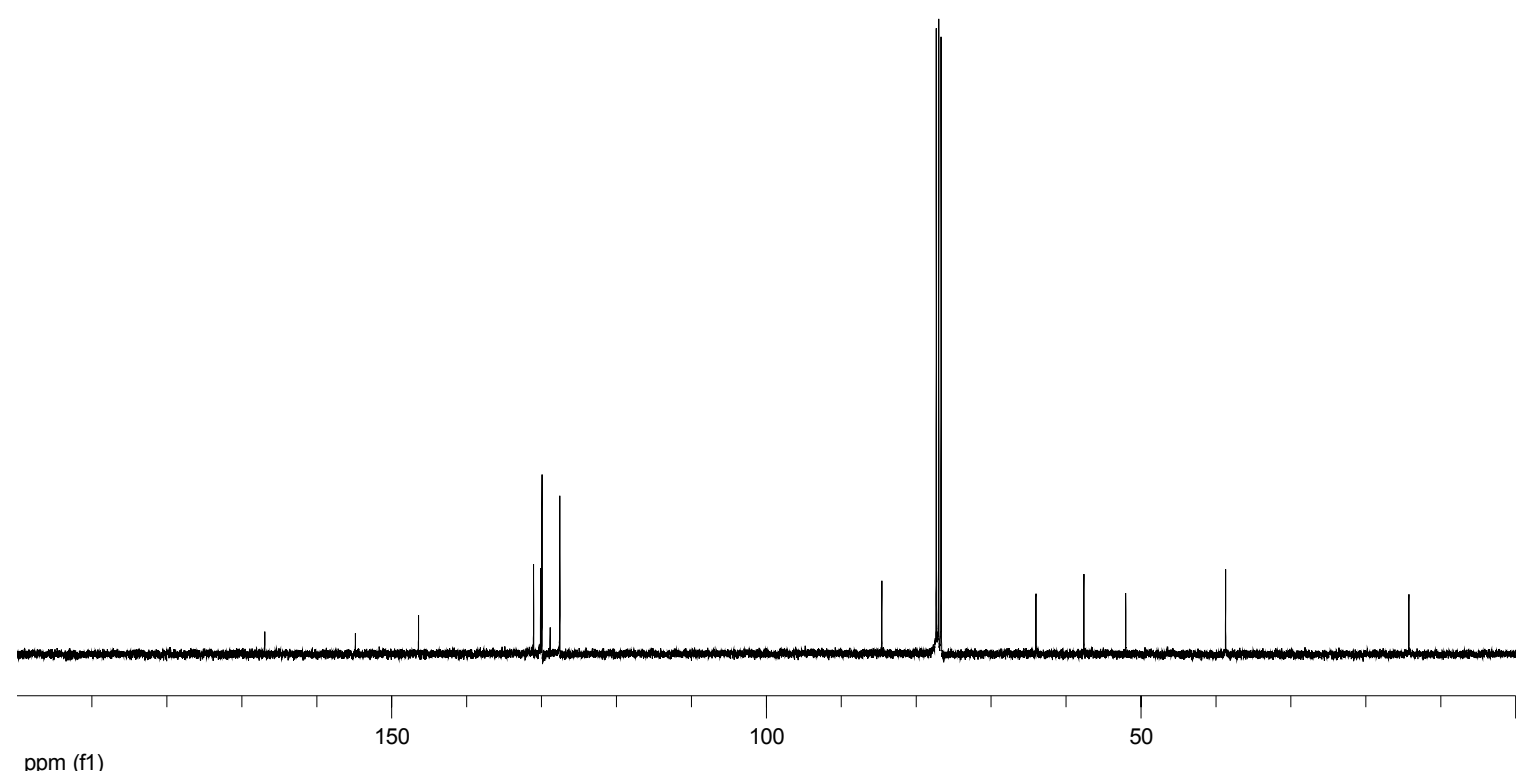

ppm (f1) 
(2c) : (1R, 2S)-trans-2-(4-Acetylphenyl)-cyclopent-3-enyl ethylcarbonate.

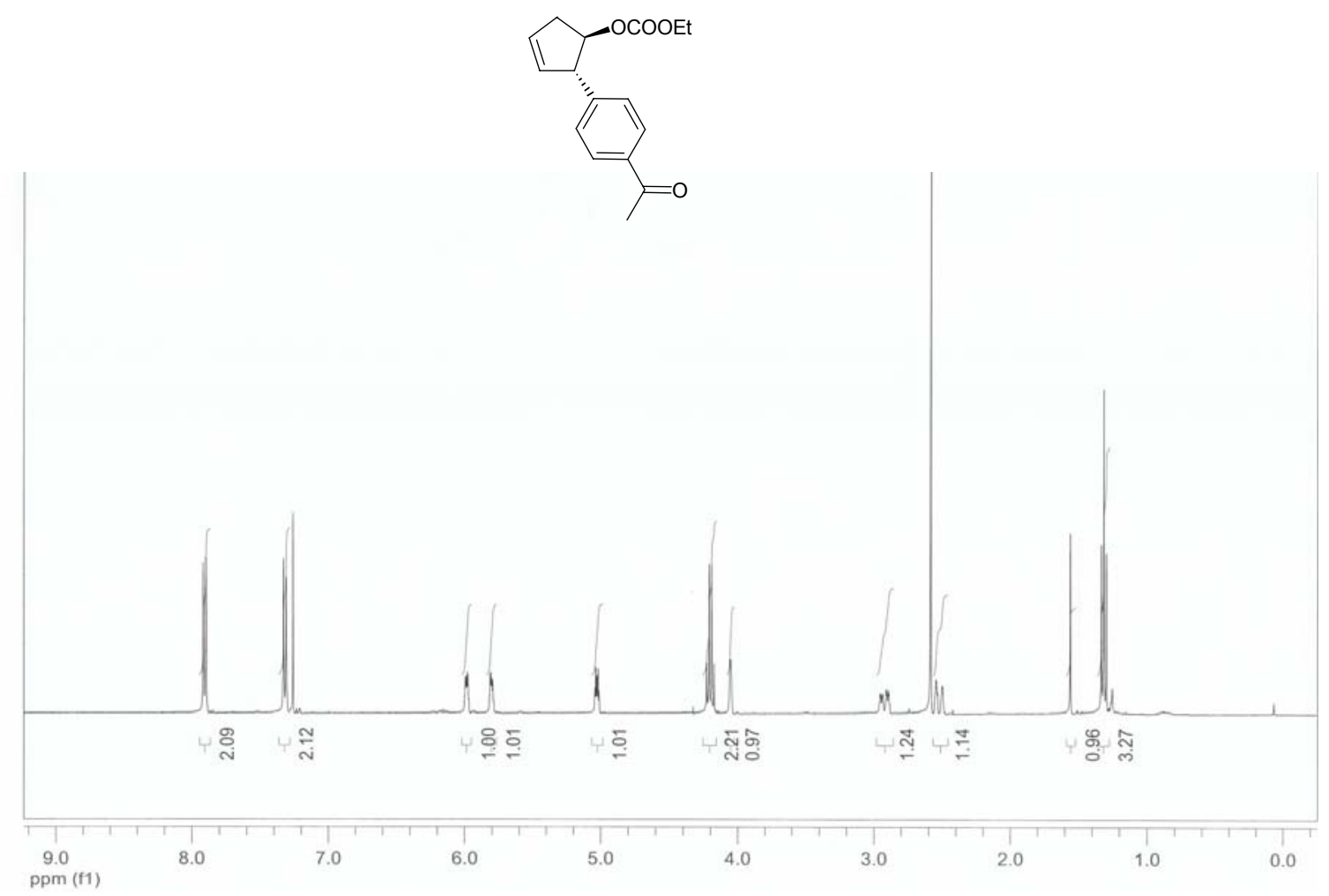

(2d) : (1R, 2S)-trans-2-(4-Trifluoromethyl-phenyl)-cyclopent-3-enyl ethylcarbonate.

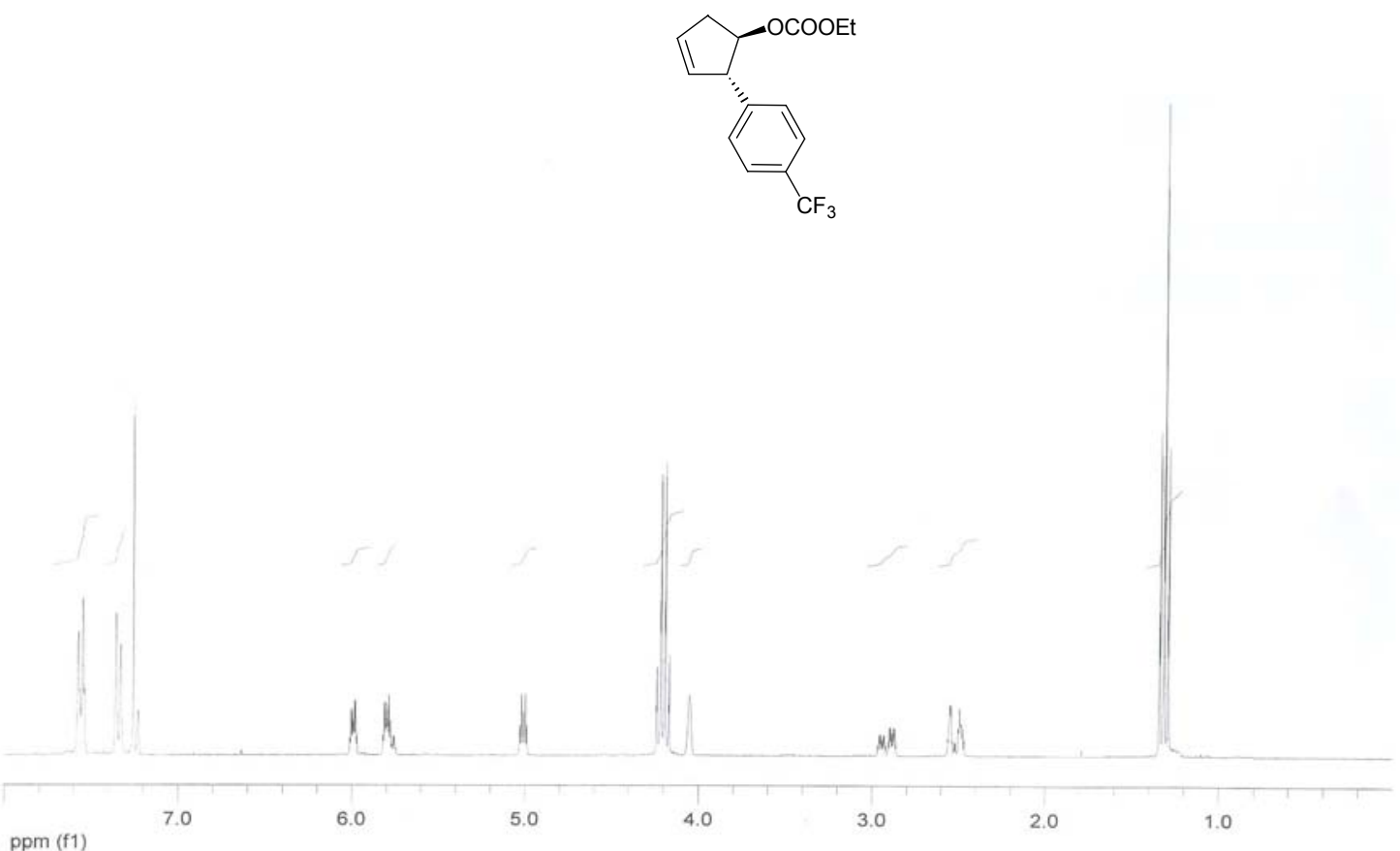


(2e) : (1R, 2S)-trans-2-(4-Fluorophenyl)-cyclopent-3-enyl ethylcarbonate.

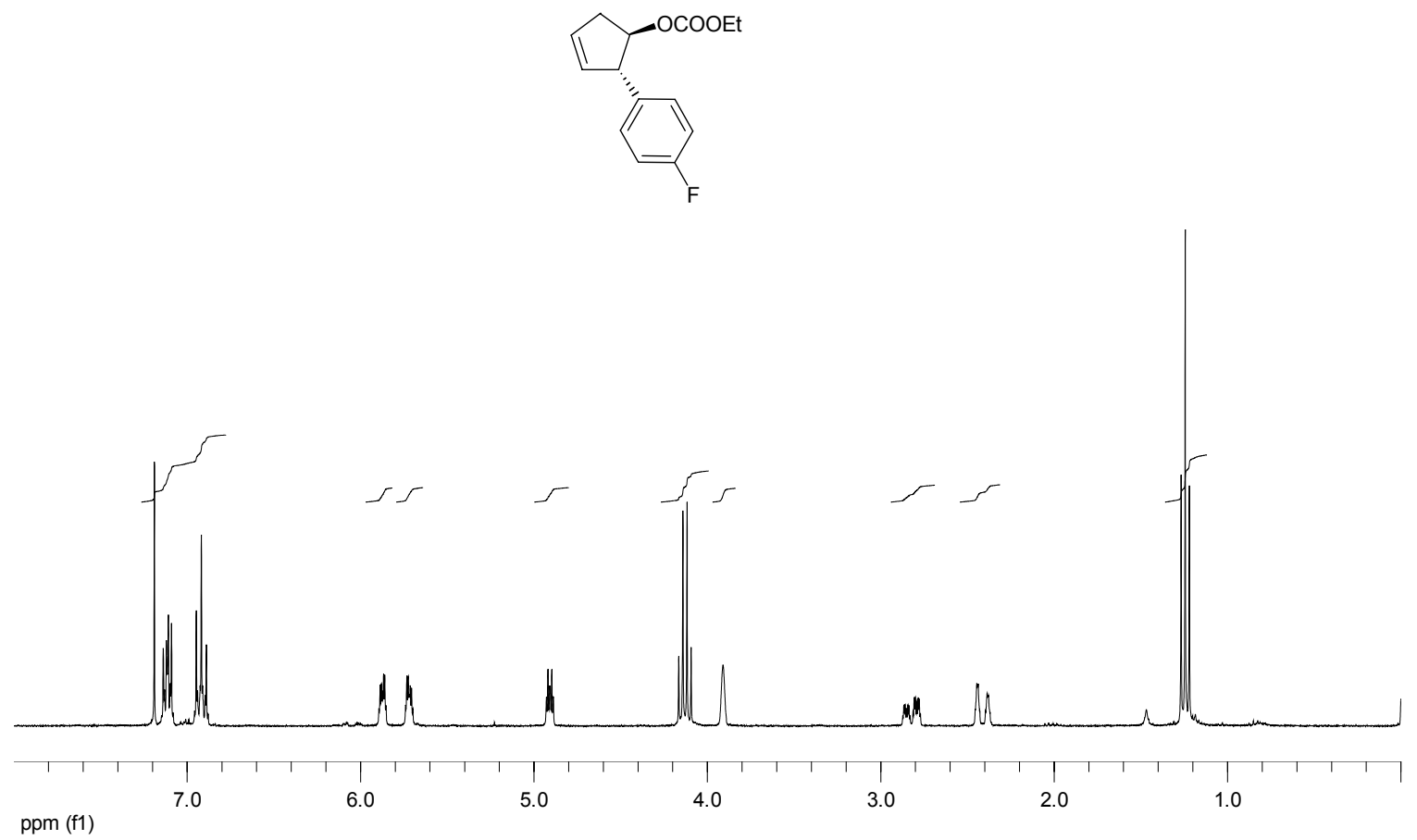

(2f) : (1R, 2S)-trans-2-(4-Chlorophenyl)-cyclopent-3-enyl ethylcarbonate.

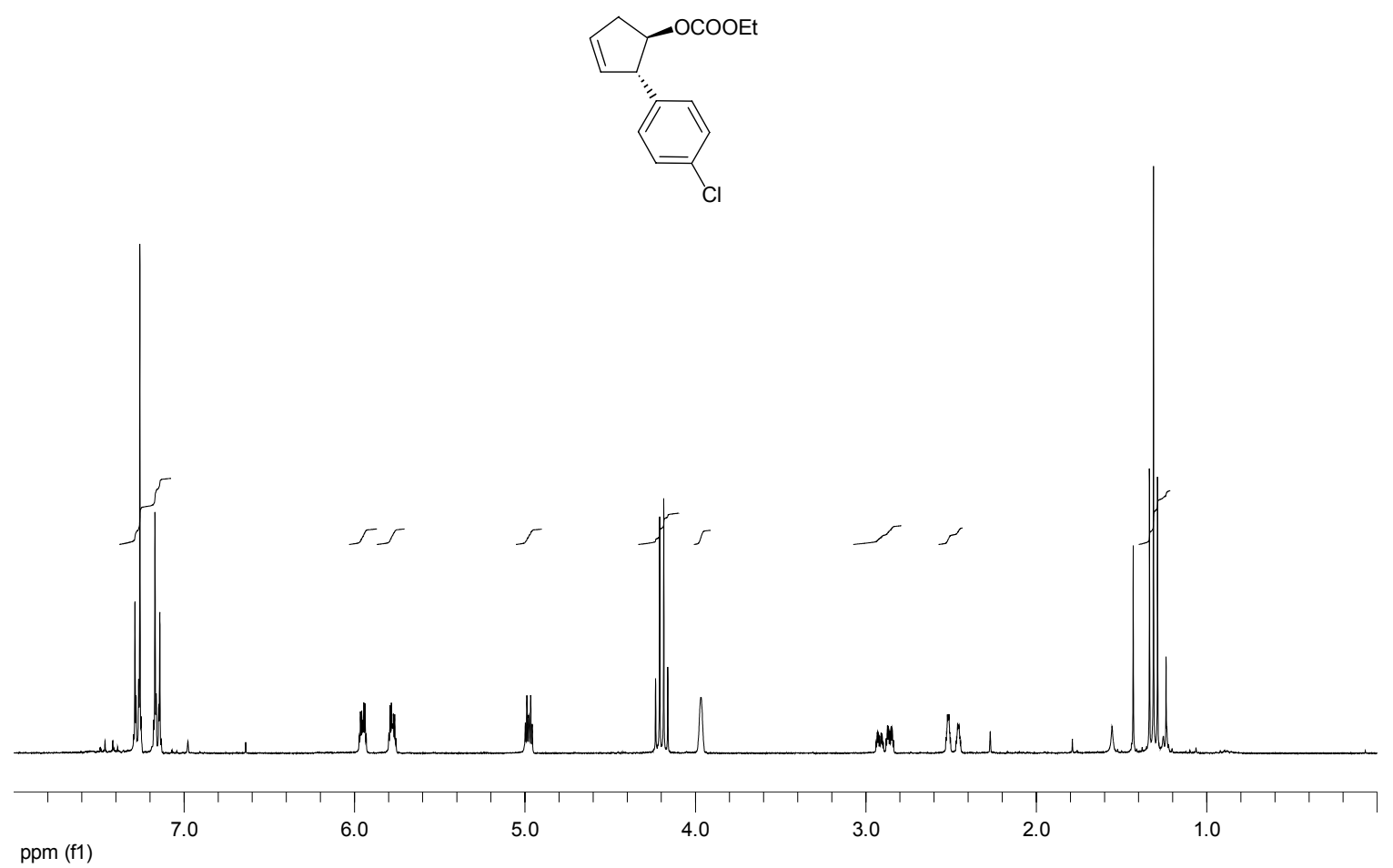


(2g) : (1R, 2S)-trans-2-(4-Bromophenyl)-cyclopent-3-enyl ethylcarbonate.

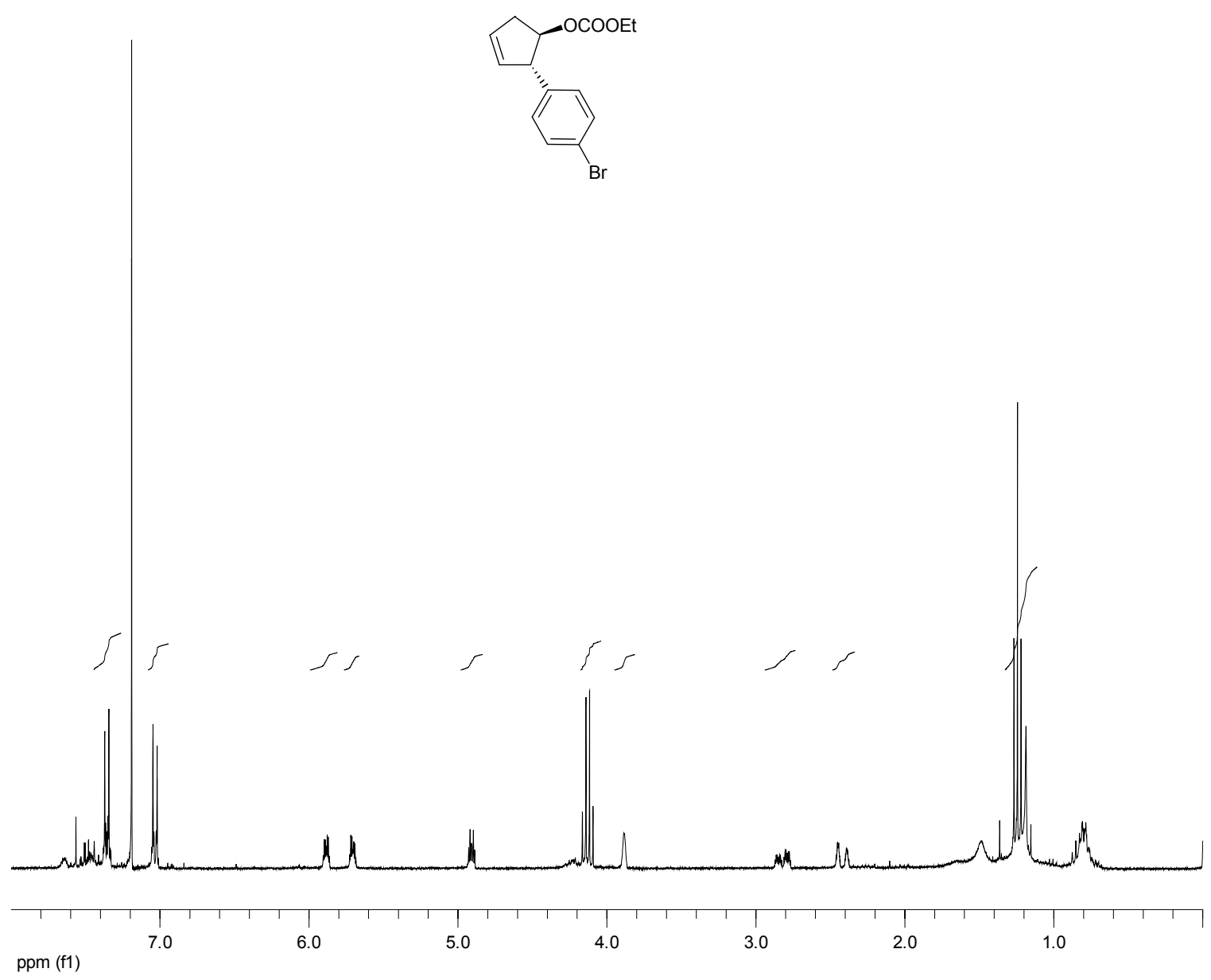


(2h) : (1R, 2S)-trans-2-(4-Methylphenyl)-cyclopent-3-enyl ethylcarbonate.

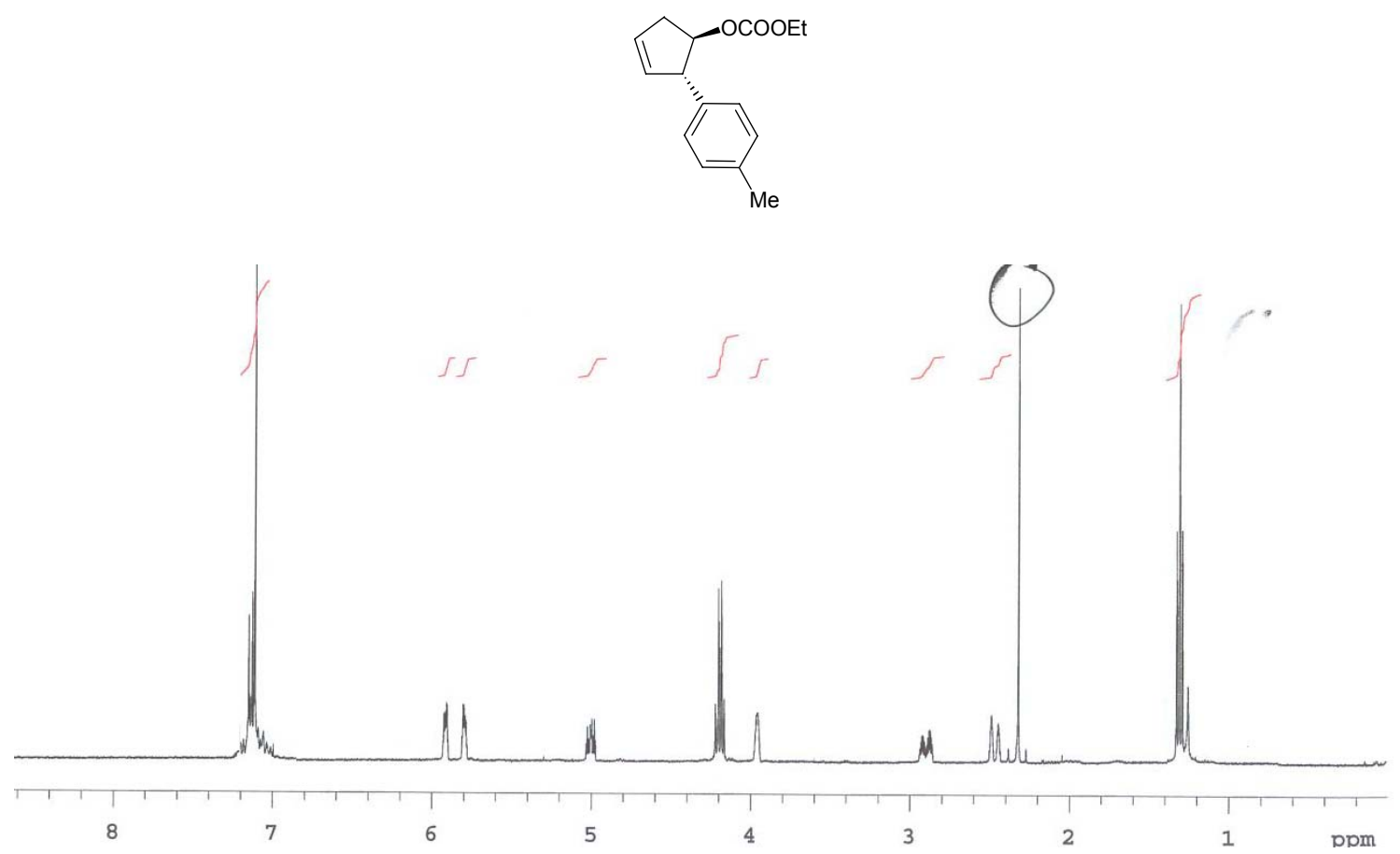

(2i) : (1R, 2S)-trans-2-(4-[t-Butoxycarbonylamino]-phenyl)-cyclopent-3-enyl ethylcarbonate.

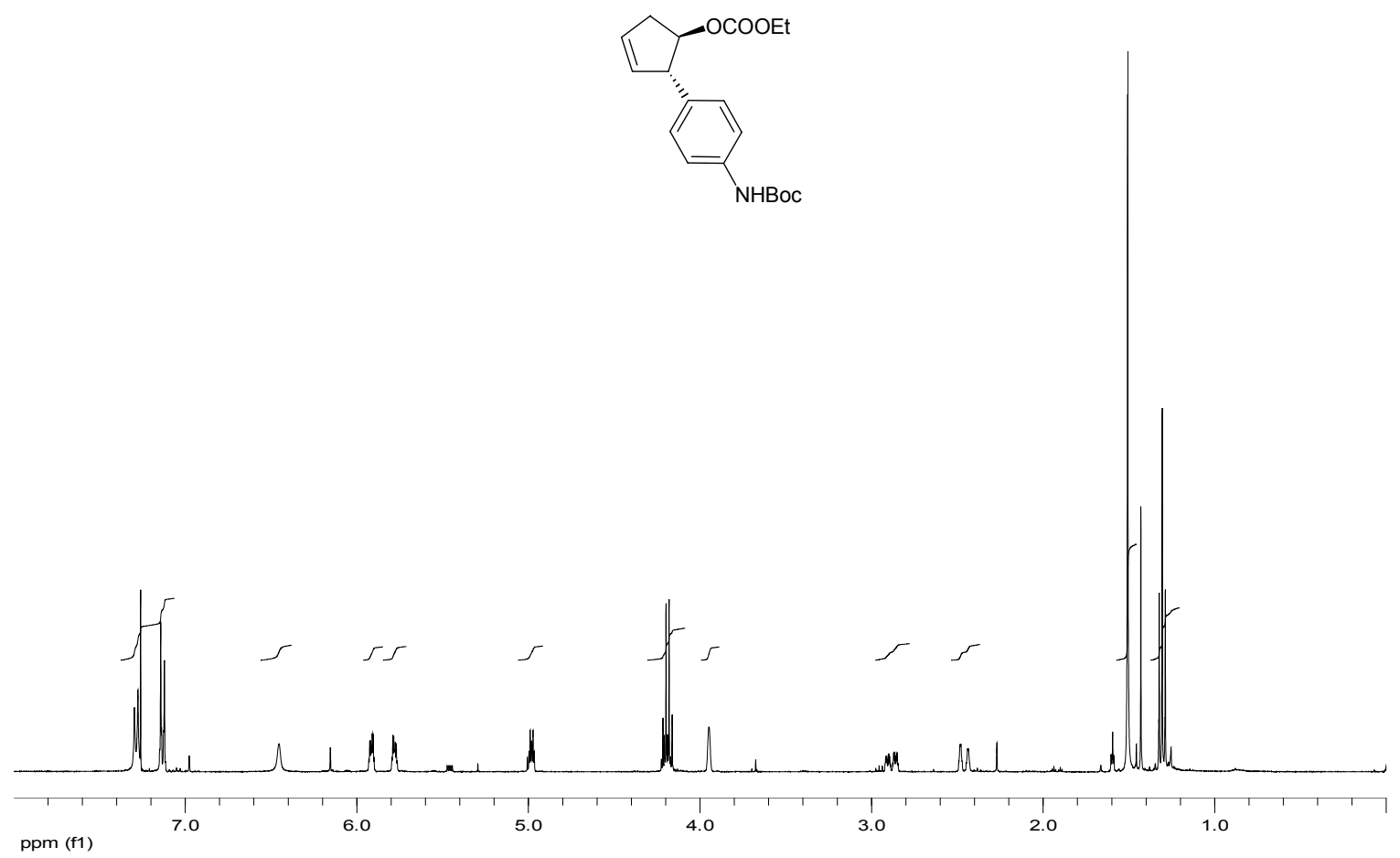


(2j) : (1R, 2S)-trans-2-(4-Methoxyphenyl)-cyclopent-3-enyl ethylcarbonate.

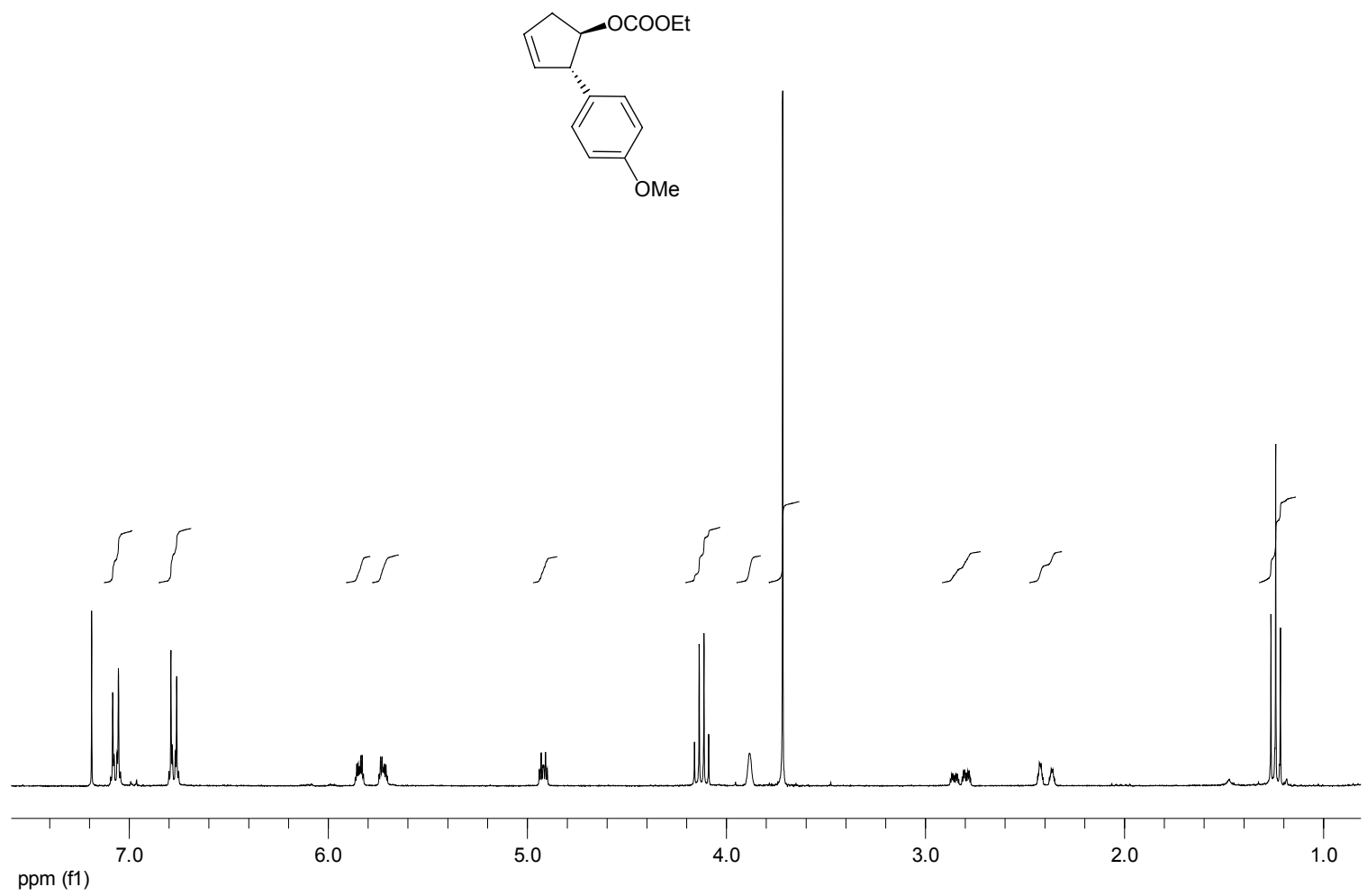

(2k) : (1R, 2S)-trans-2-(3-Methoxyphenyl)-cyclopent-3-enyl ethylcarbonate.

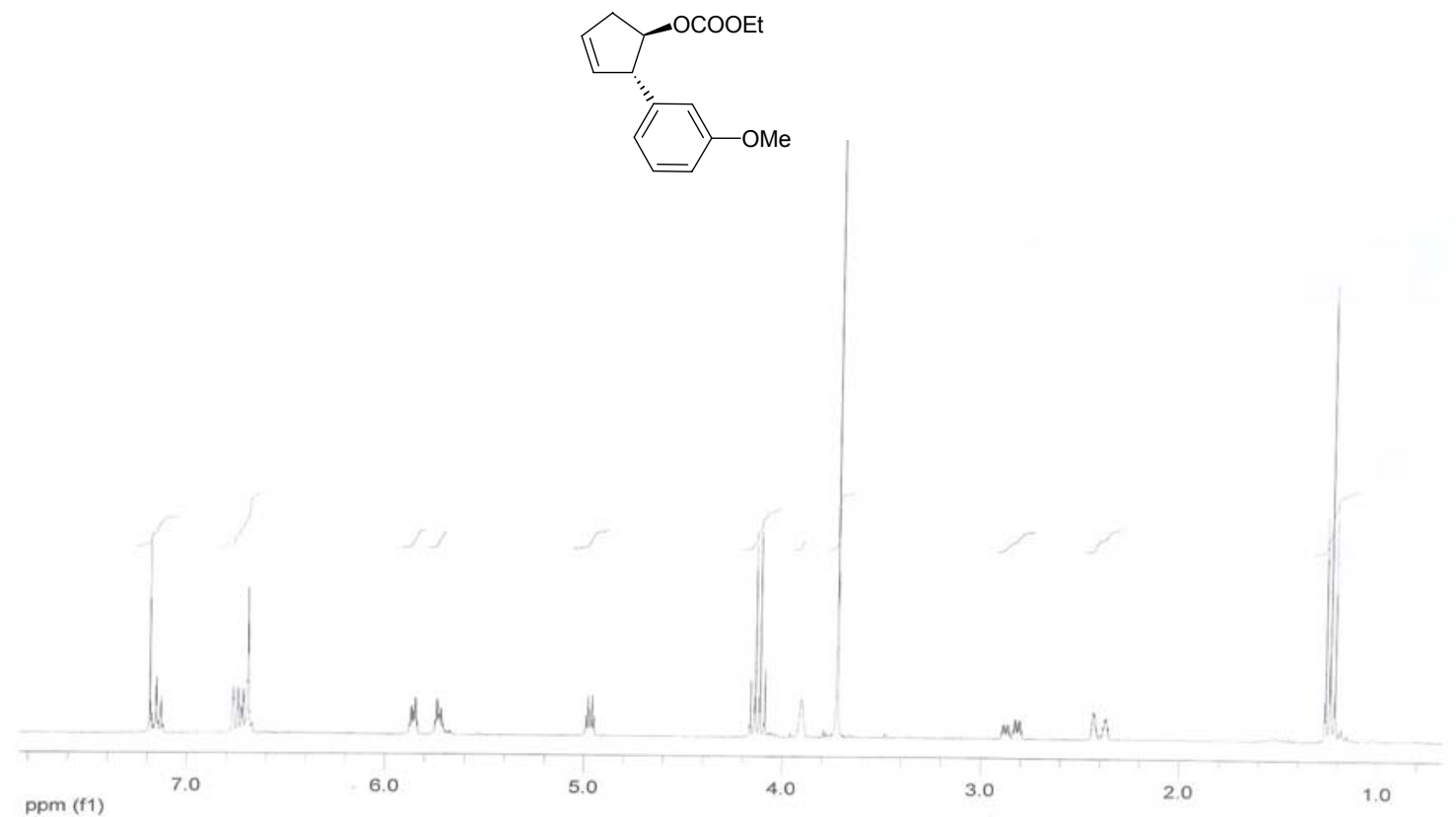


(2I) : (1R, 2S)-trans-2-(3-Chlorophenyl)-cyclopent-3-enyl ethylcarbonate.

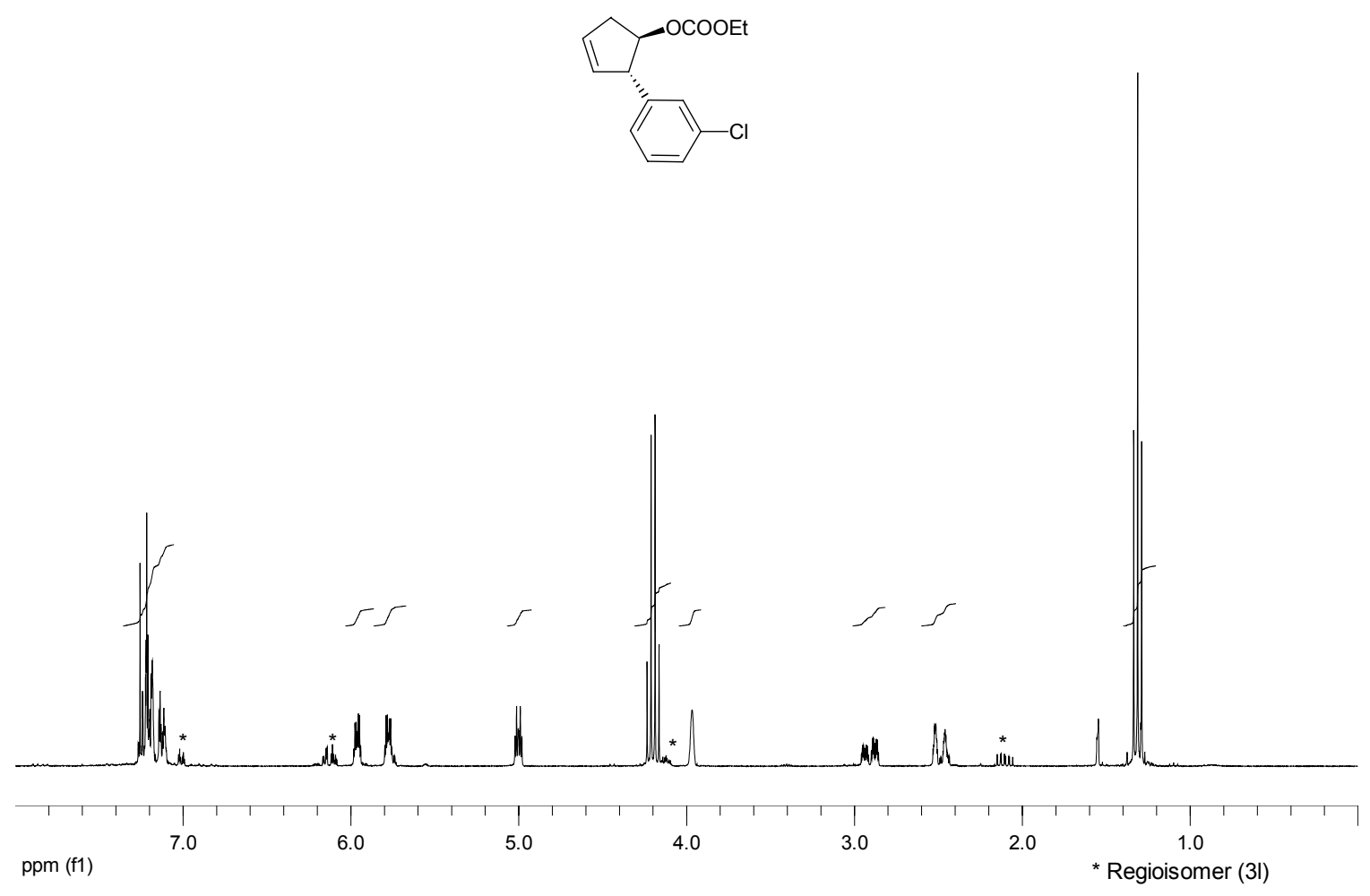

(2m) : (1R, 2S)-trans-2-(3-Methylphenyl)-cyclopent-3-enyl ethylcarbonate.

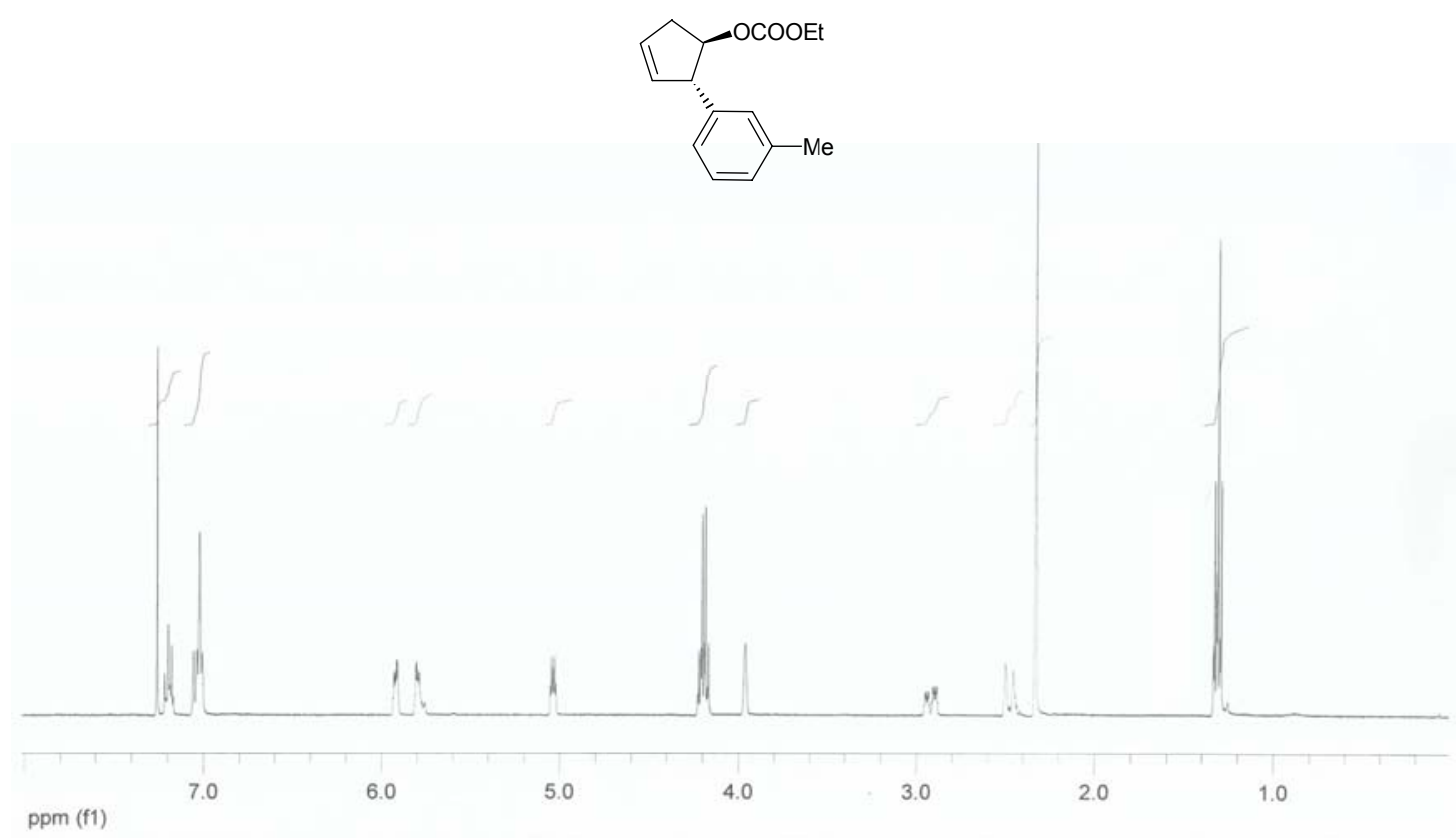


(2n) : (1R, 2S)-trans-2-(2-Naphthalenyl)- cyclopent-3-enyl ethylcarbonate.

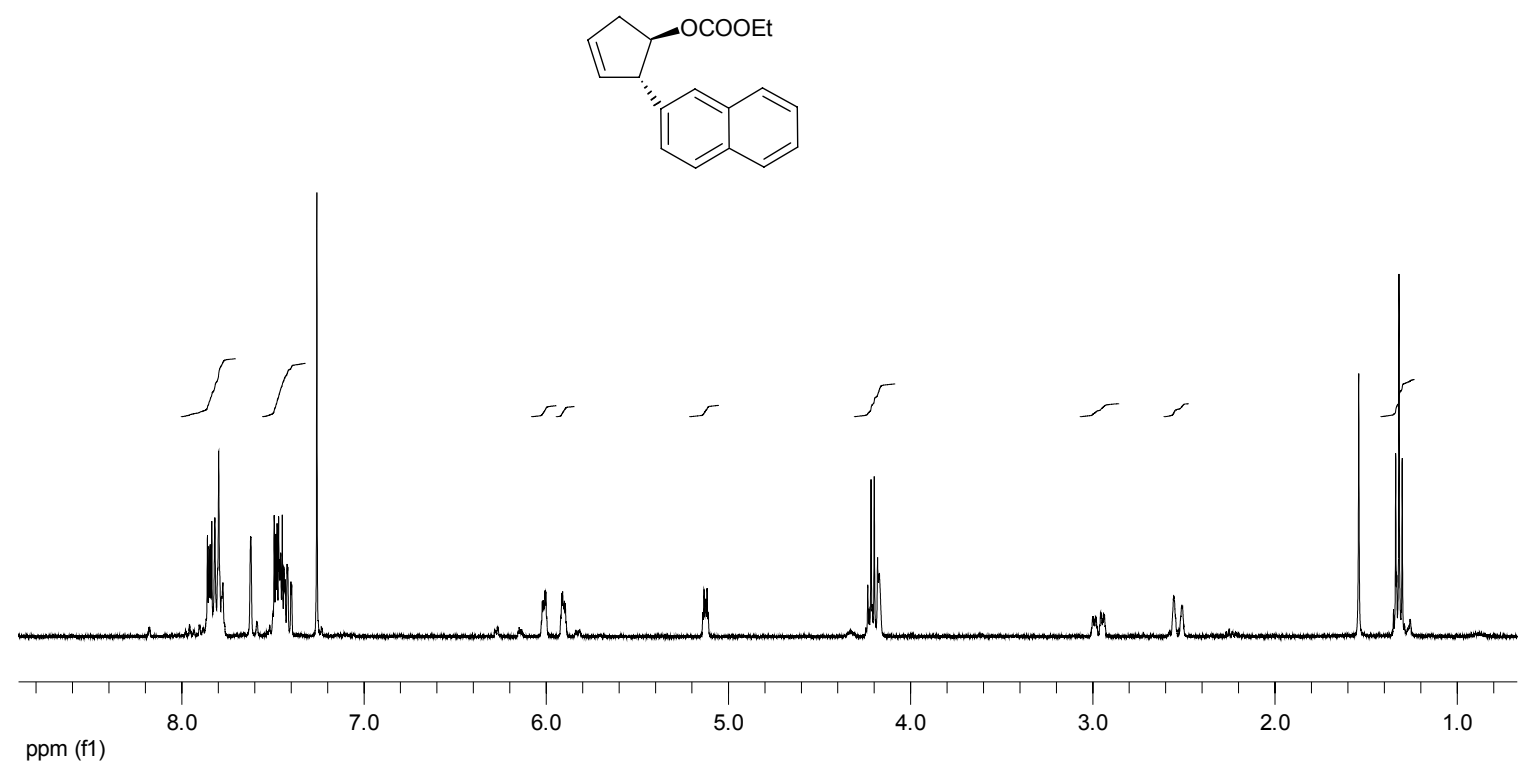

(2o) : (1R, 2S)-trans-2-(1-Naphthalenyl)- cyclopent-3-enyl ethylcarbonate.

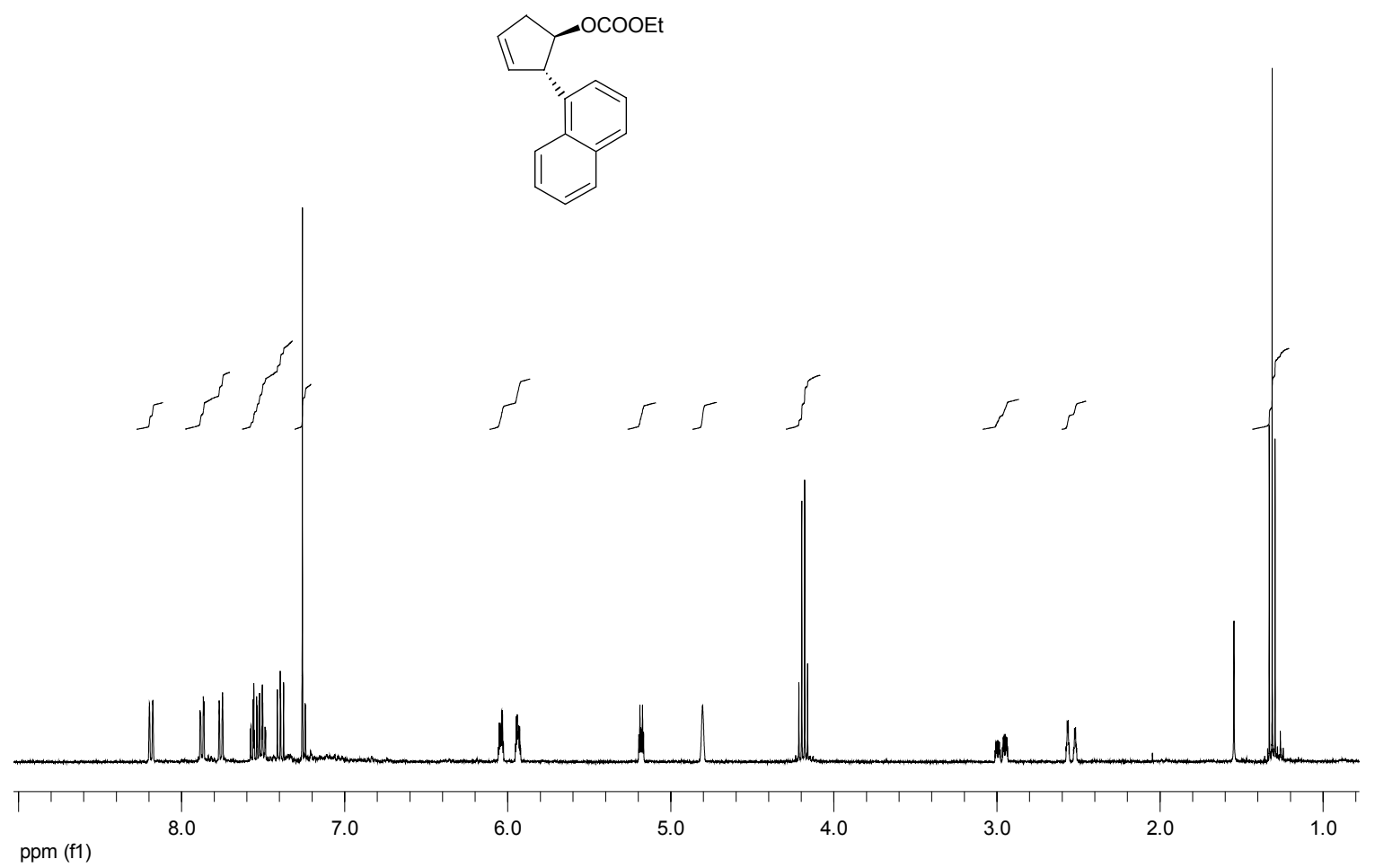


(2p) : (1R, 2S)-trans-2-(2-Methylphenyl)-cyclopent-3-enyl ethylcarbonate.

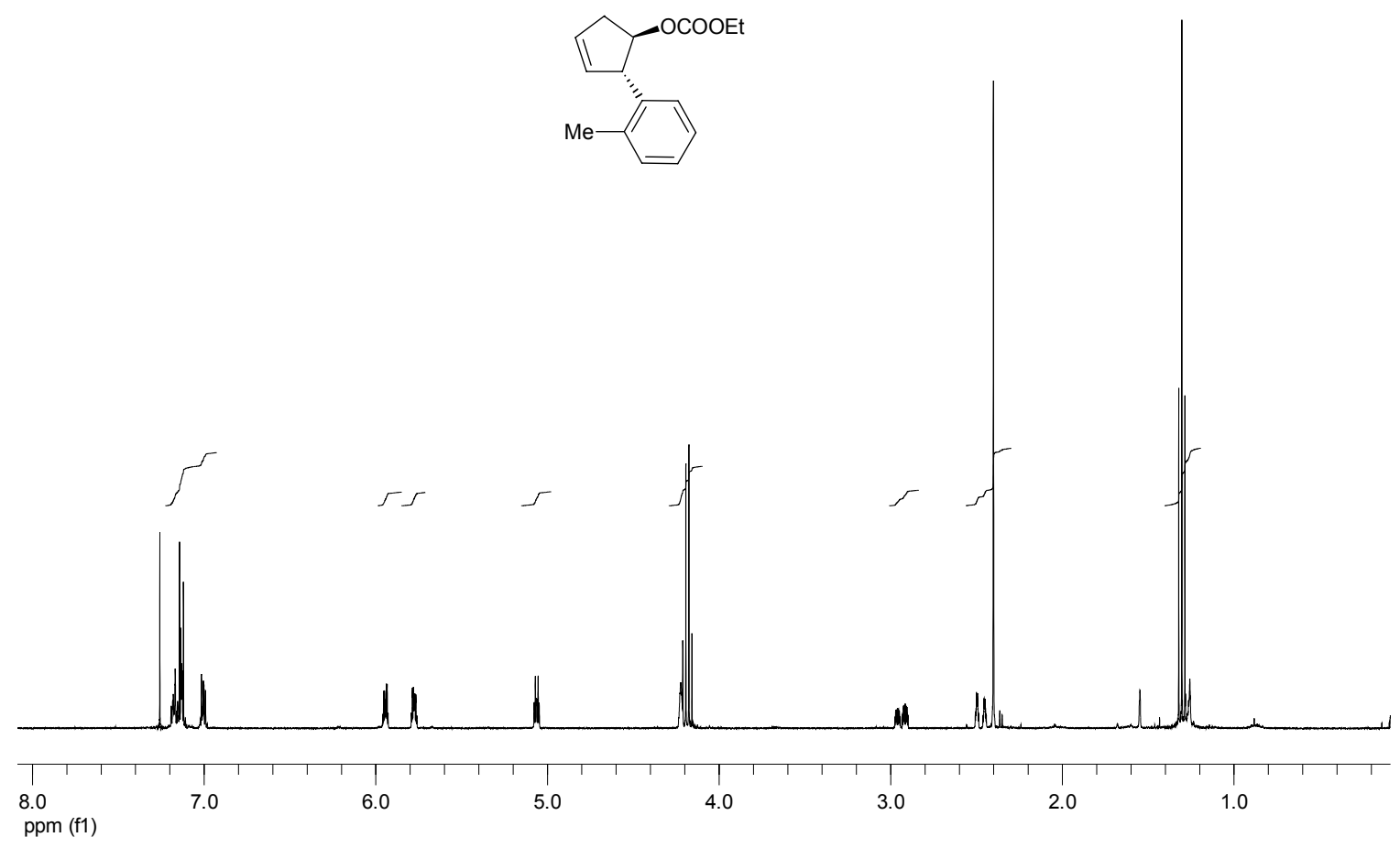


(5b) : (1R, 4R)-trans-4-(4-Methoxycarbonylphenyl)-cyclopent-2-enol.
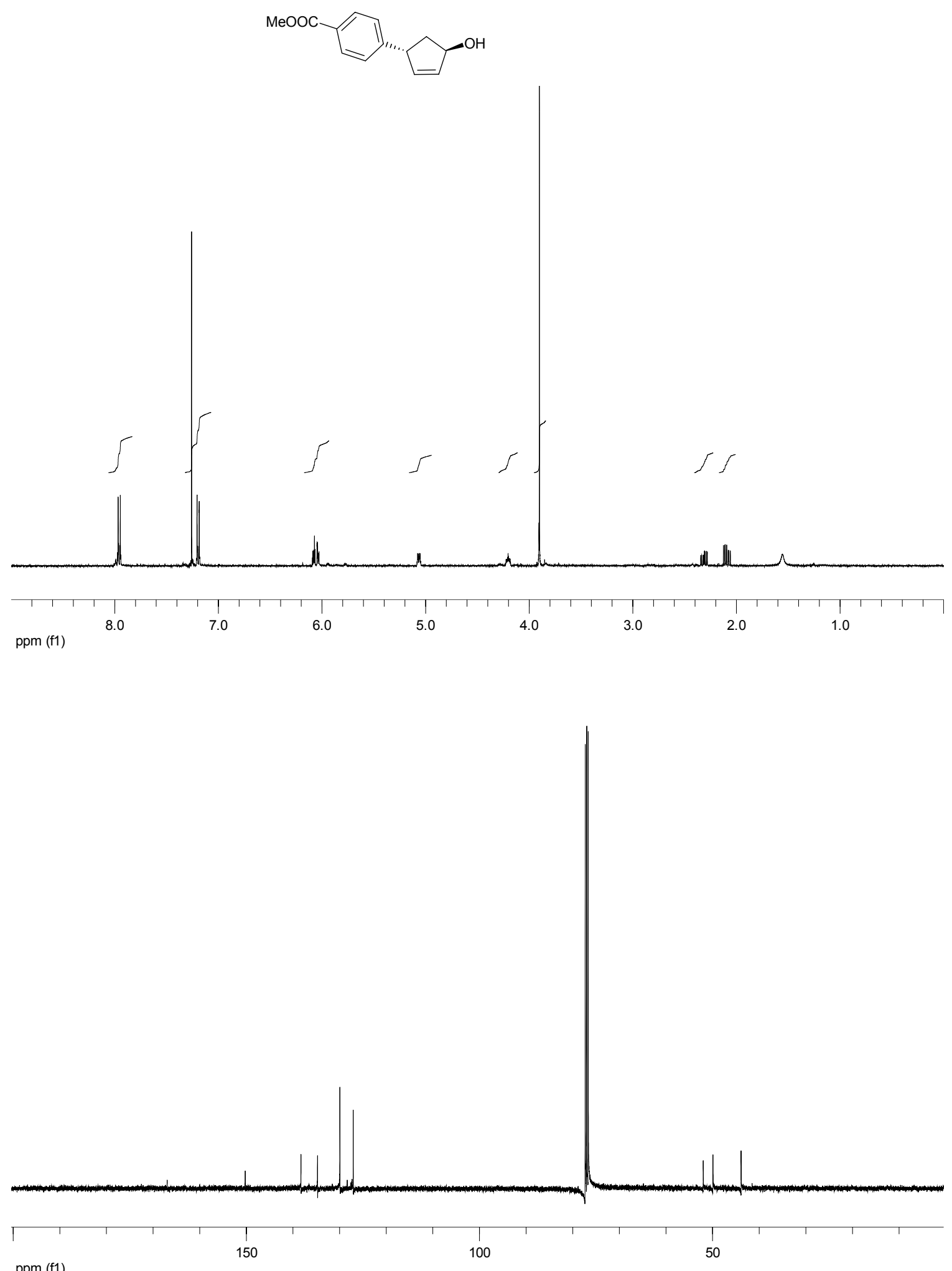
(5c) : (1R, 4R)-trans-4-(4-Acetylphenyl)-cyclopent-2-enol
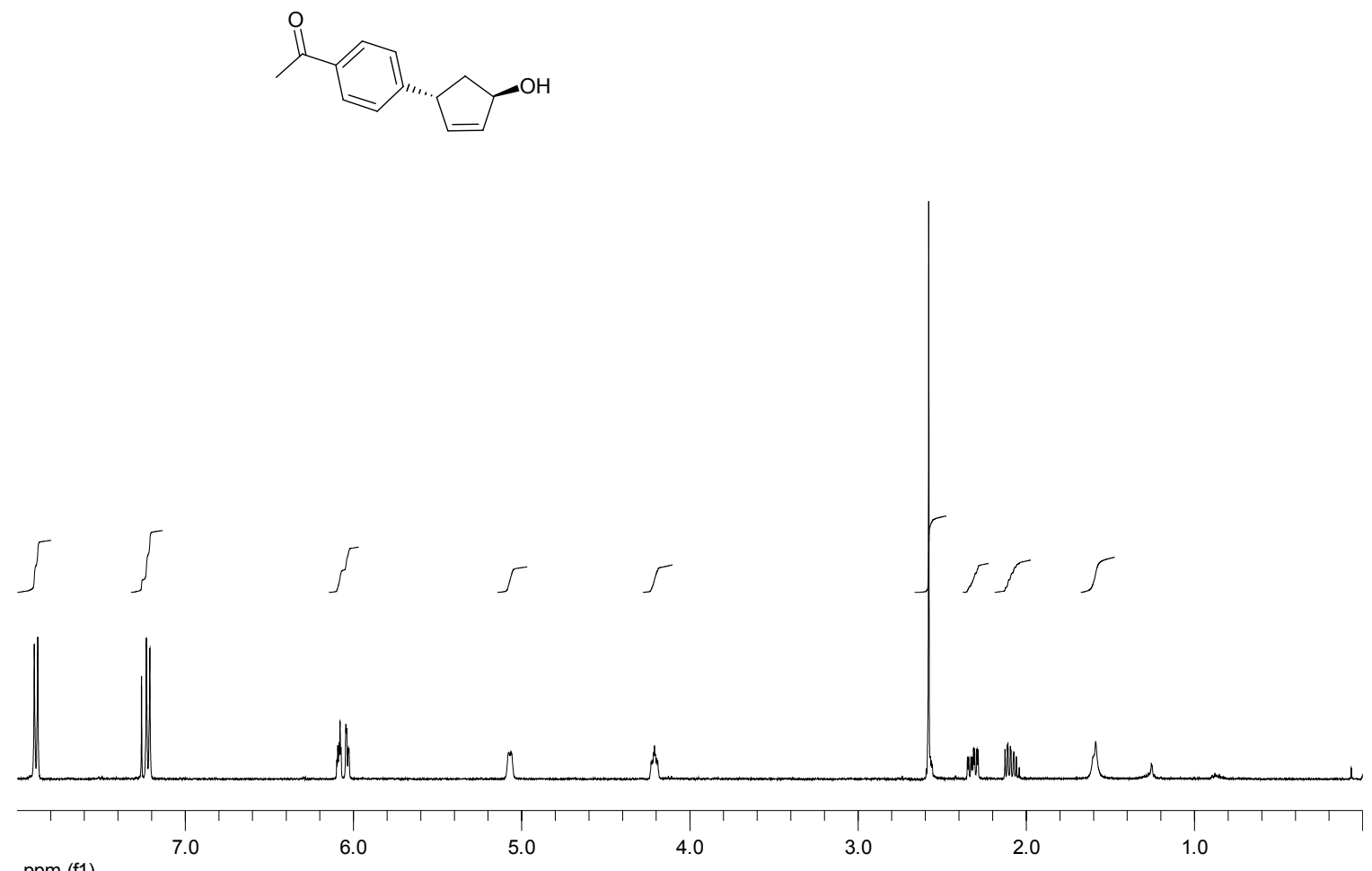

ppm (f1)

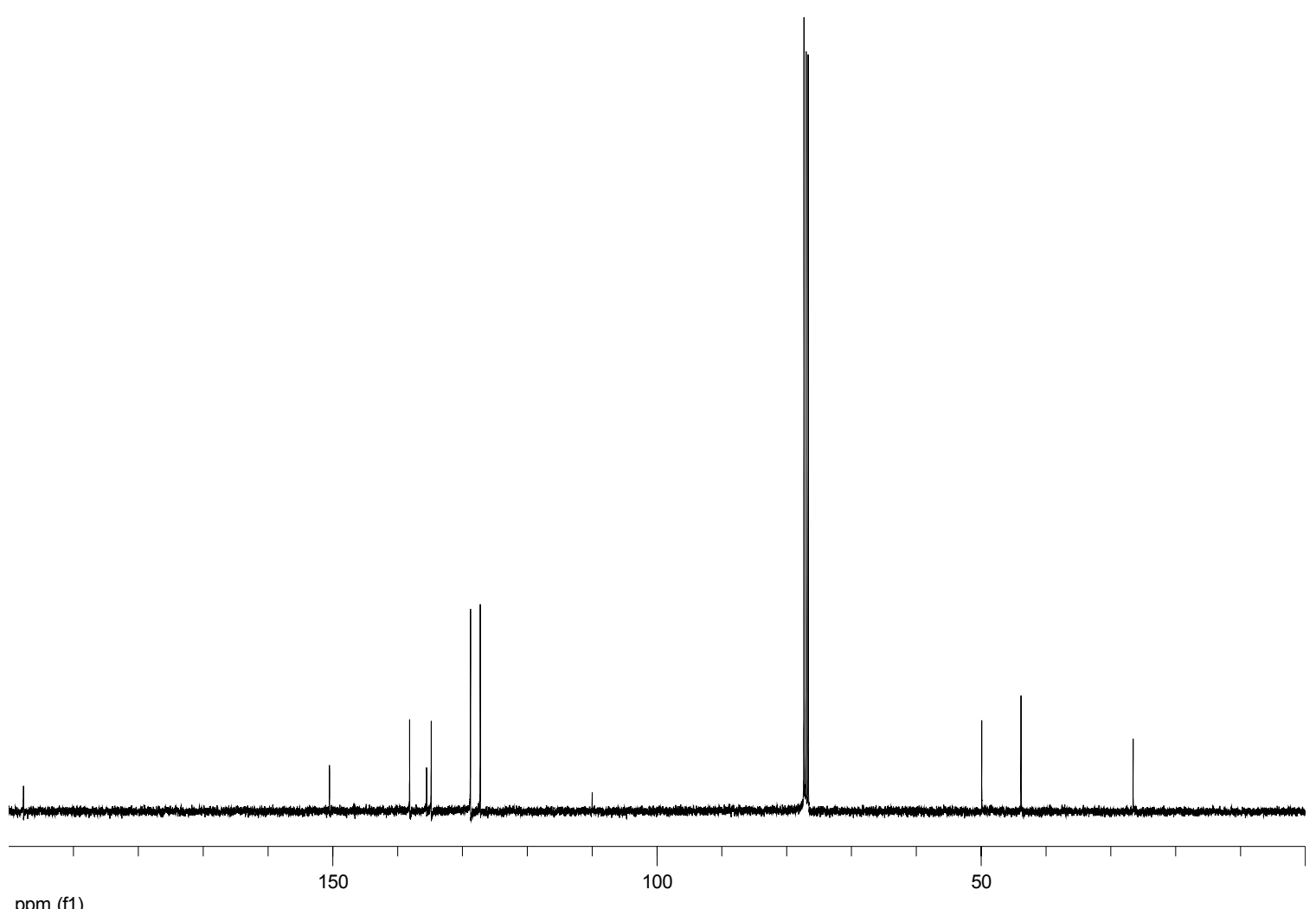

ppm (f1) 
(5e) : (1R, 4R)-trans-2-(4-Fluorophenyl)-cyclopent-2-enol.

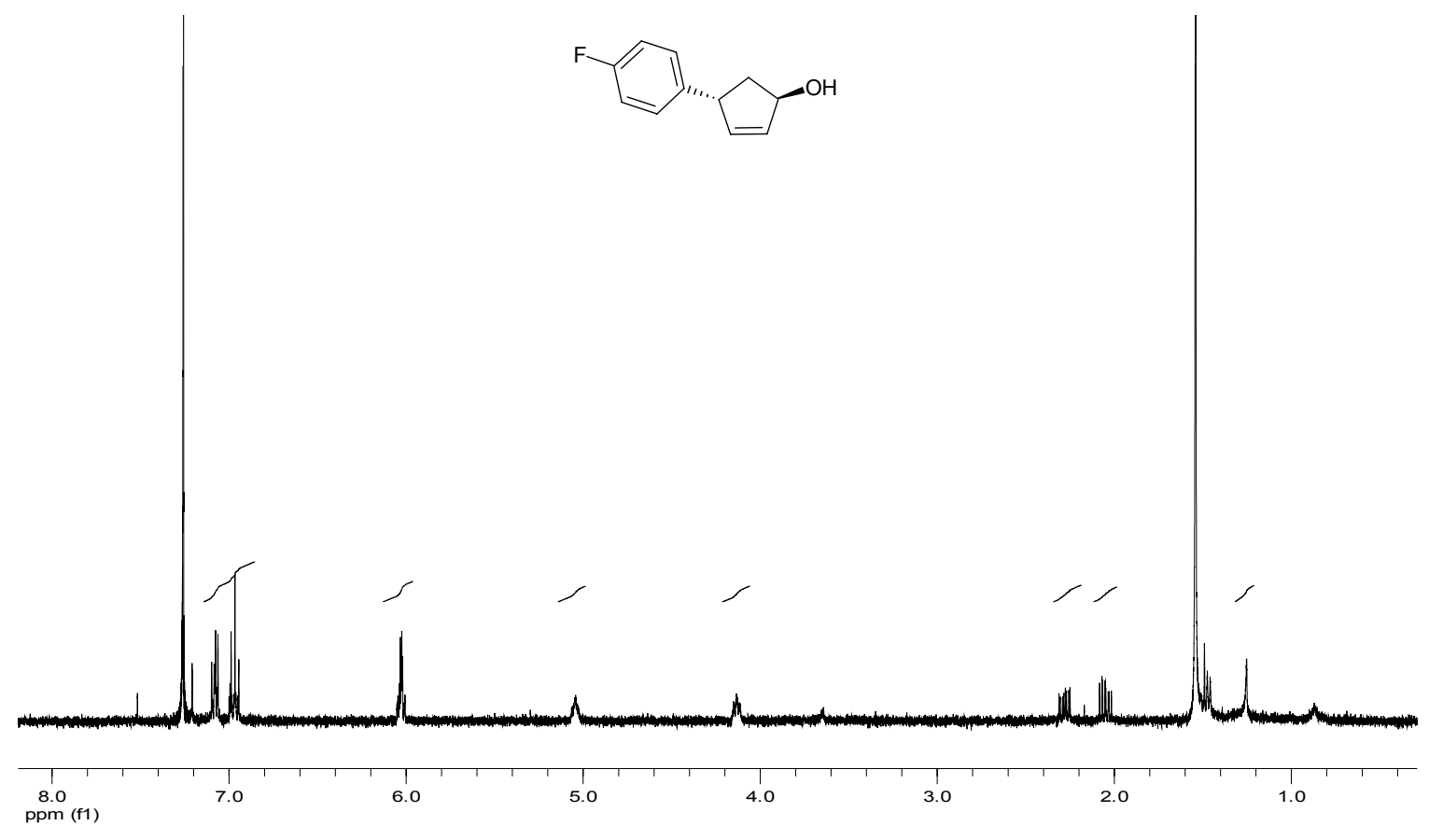

(5f) : (1R, 4R)-trans-2-(4-Chlorophenyl)-cyclopent-2-enol.

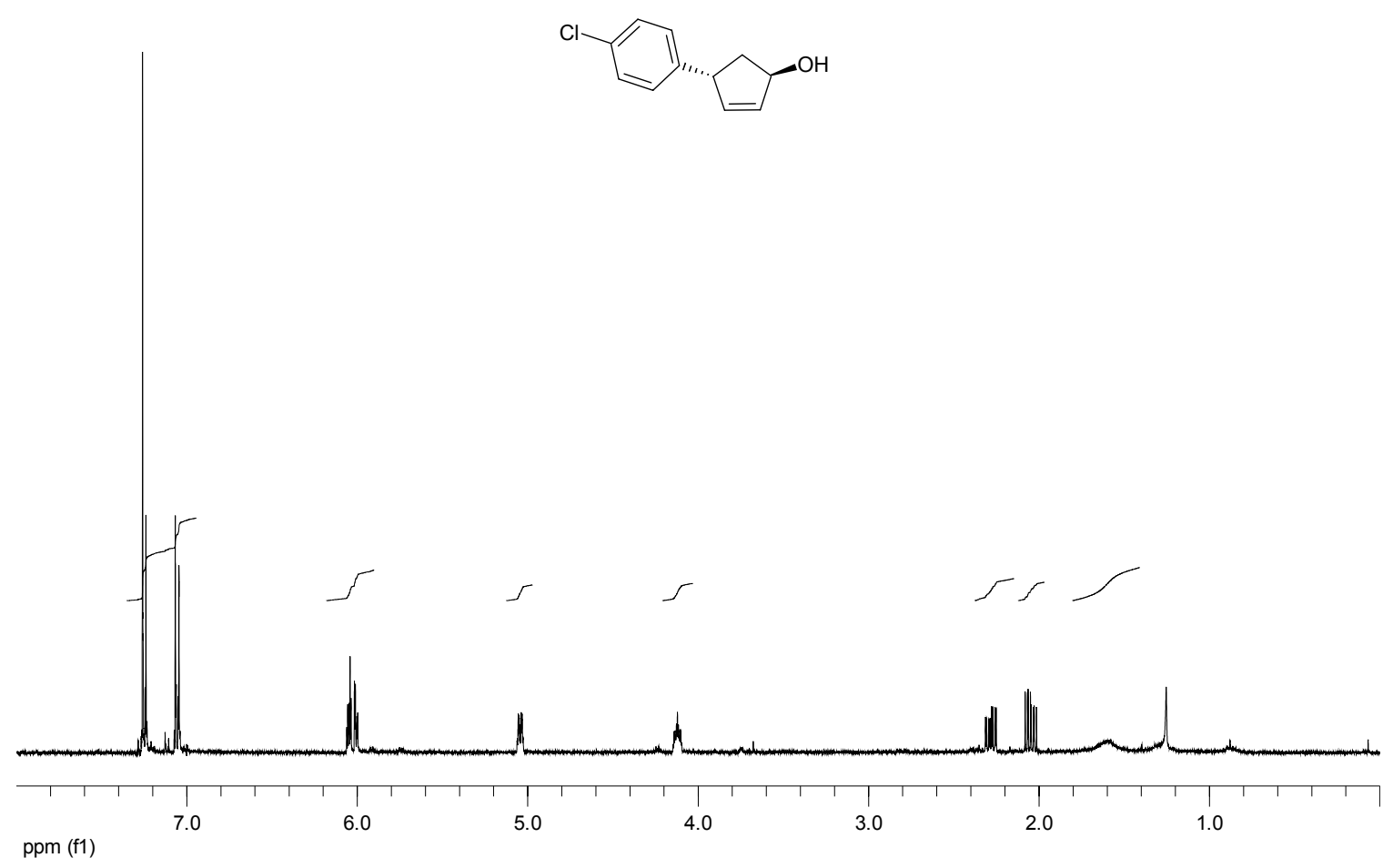


(5g) : (1R, 4R)-trans-2-(4-Bromophenyl)-cyclopent-2-enol.
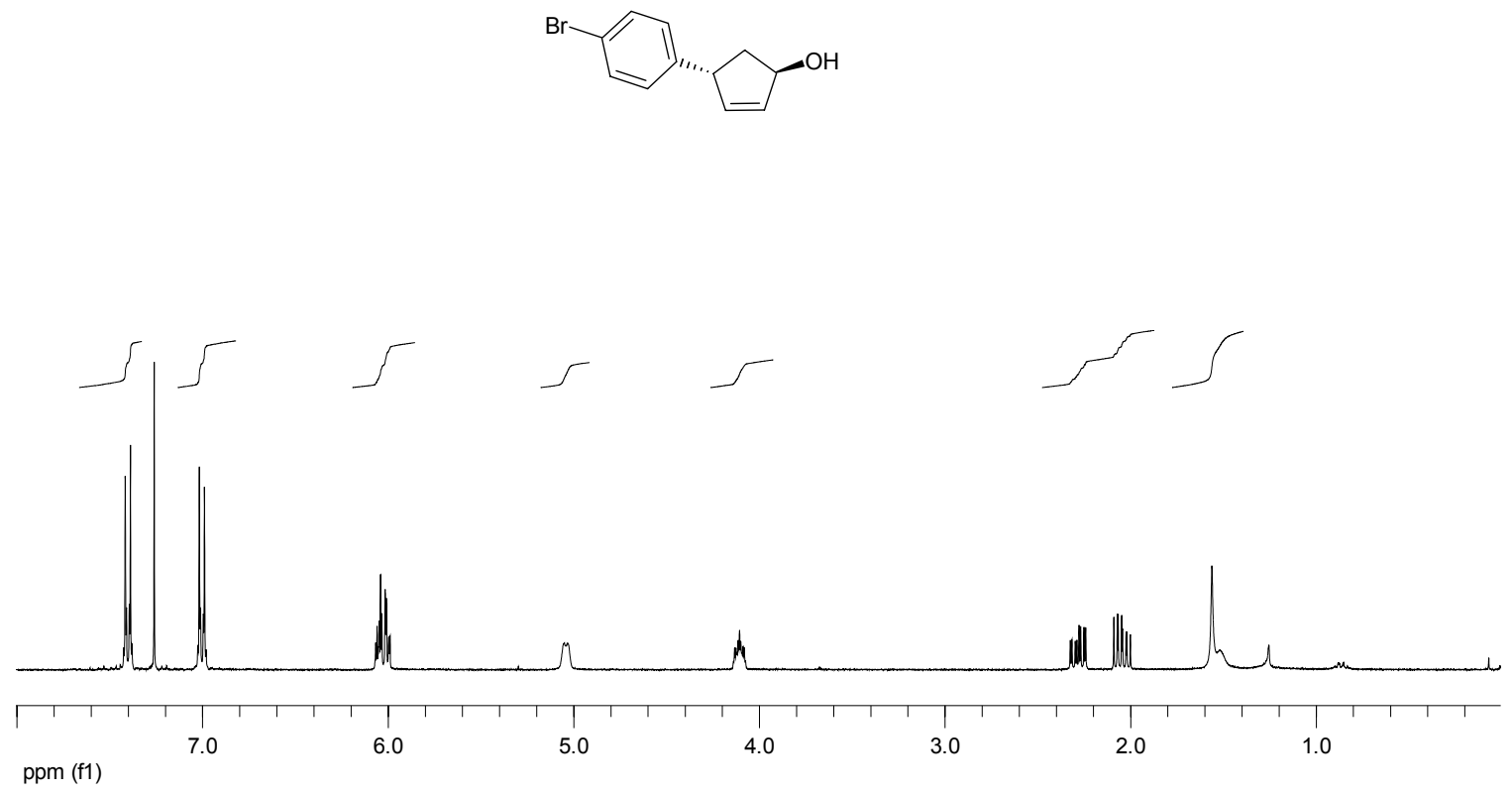

(5j) : (1R, 4R)-trans-4-(4-Methoxyphenyl)-cyclopent-2-enol.

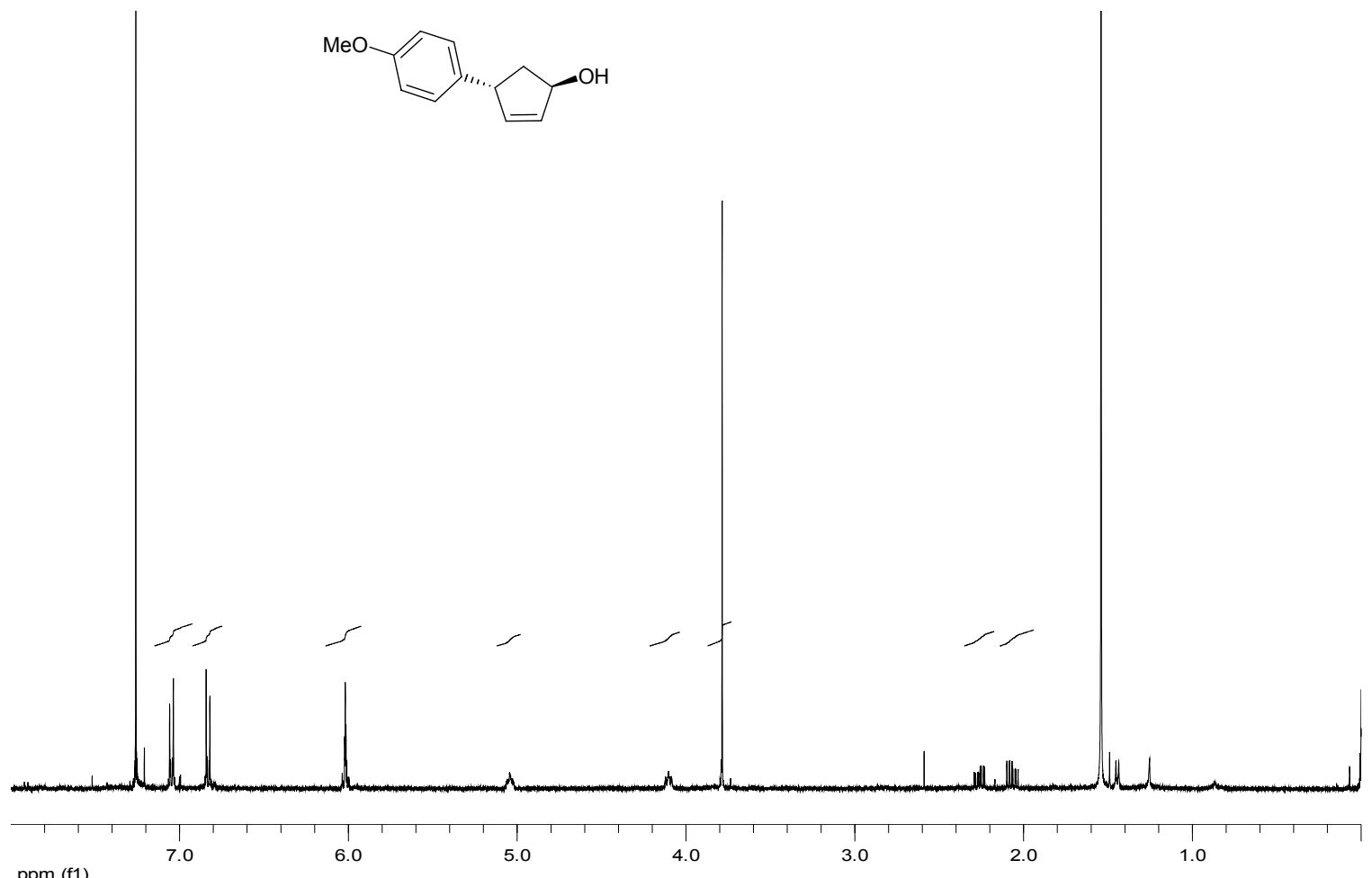


(5k) : (1R, 4R)-trans-4-(3-Methoxyphenyl)-cyclopent-2-enol.

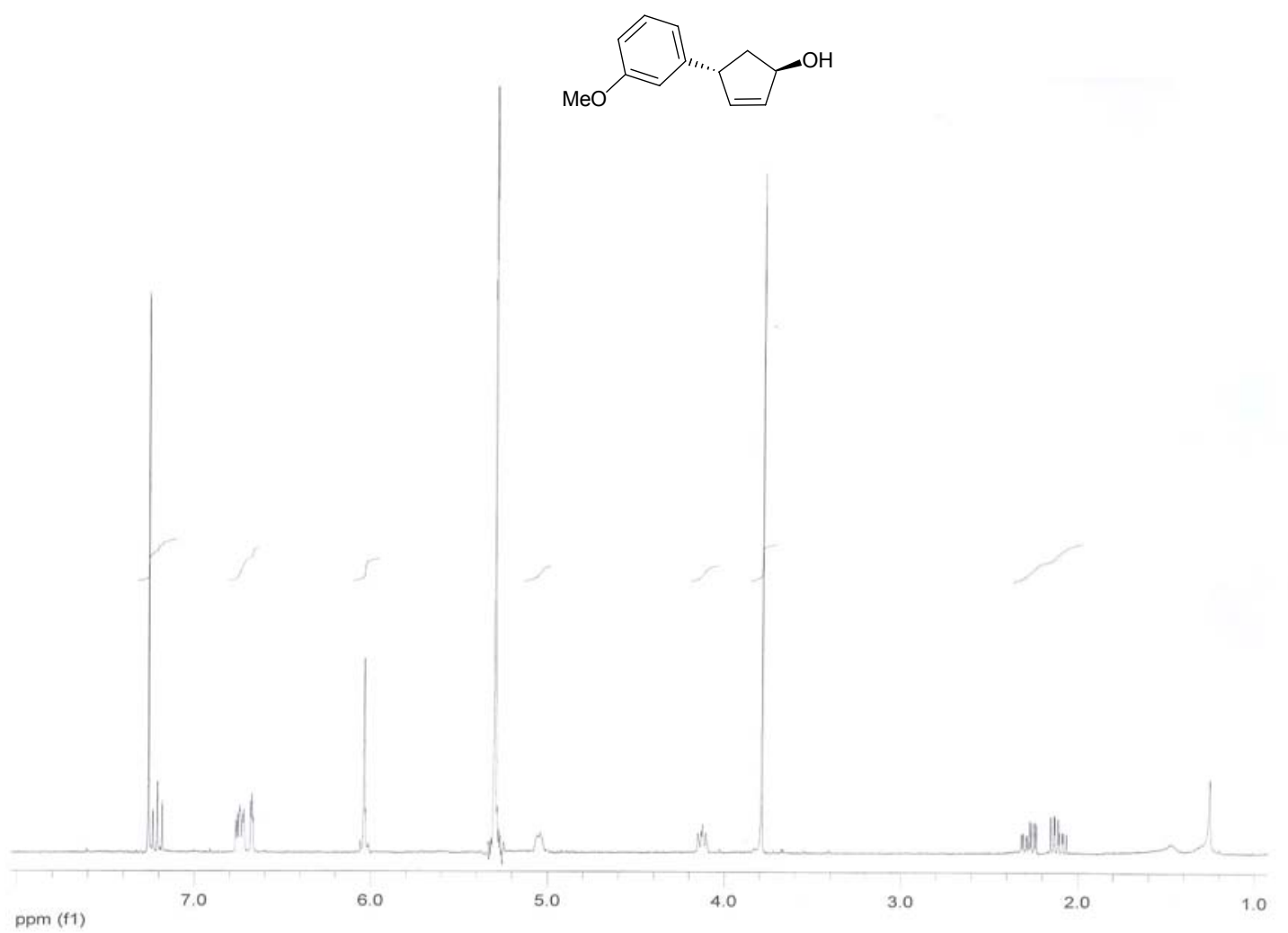


(5l) : (1R, 4R)-trans-4-(3-Chlorophenyl)-cyclopent-2-enol.<smiles>OC1C=C[C@H](c2cccc(Cl)c2)C1</smiles>

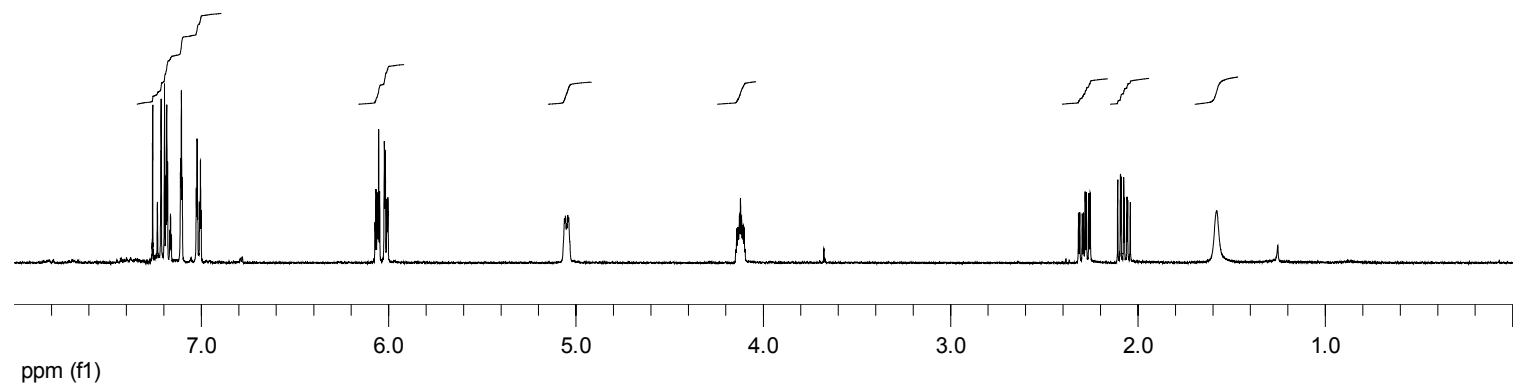

ppm (f1)

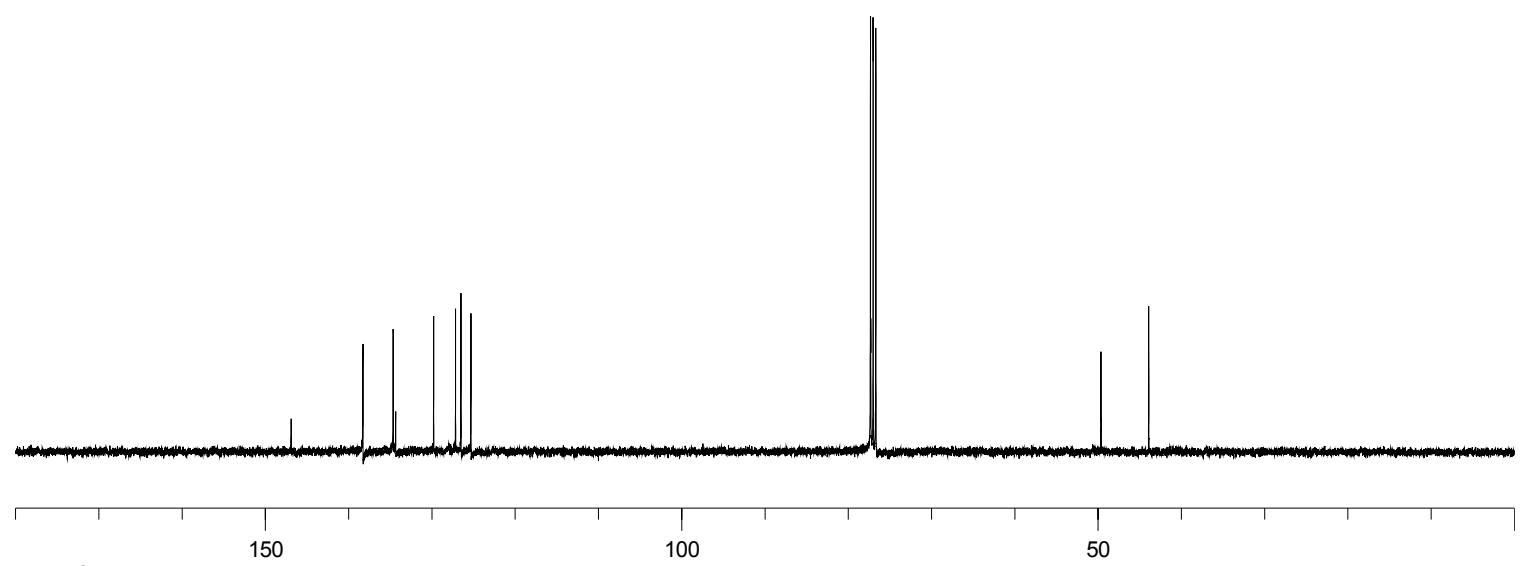

ppm (f1) 
(5m) : (1R, 4R)-trans-4-(3-Methylphenyl)-cyclopent-2-enol.
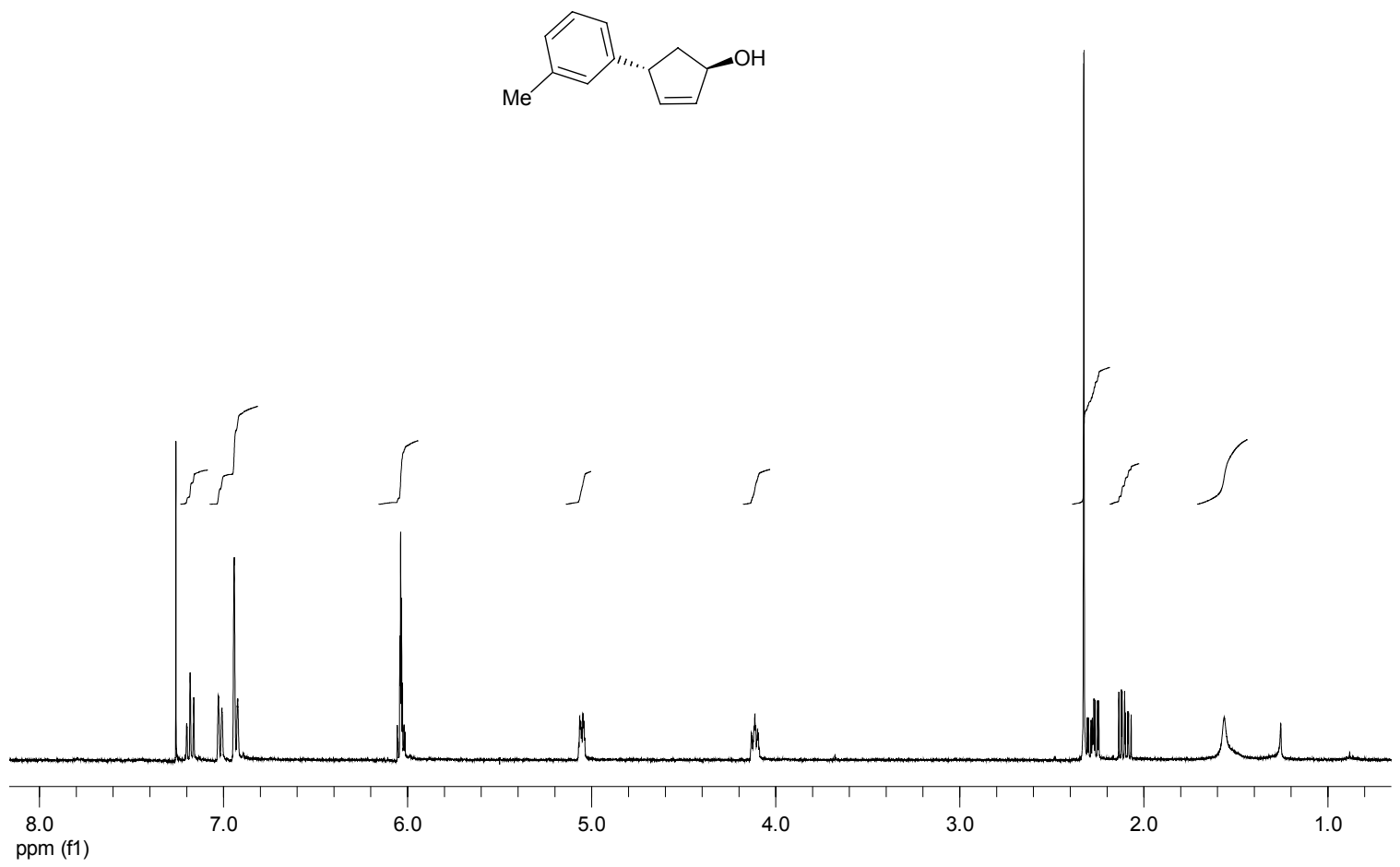

(5n) : (1R, 4R)-trans-4-(2-Naphthalenyl)-cyclopent-2-enol.
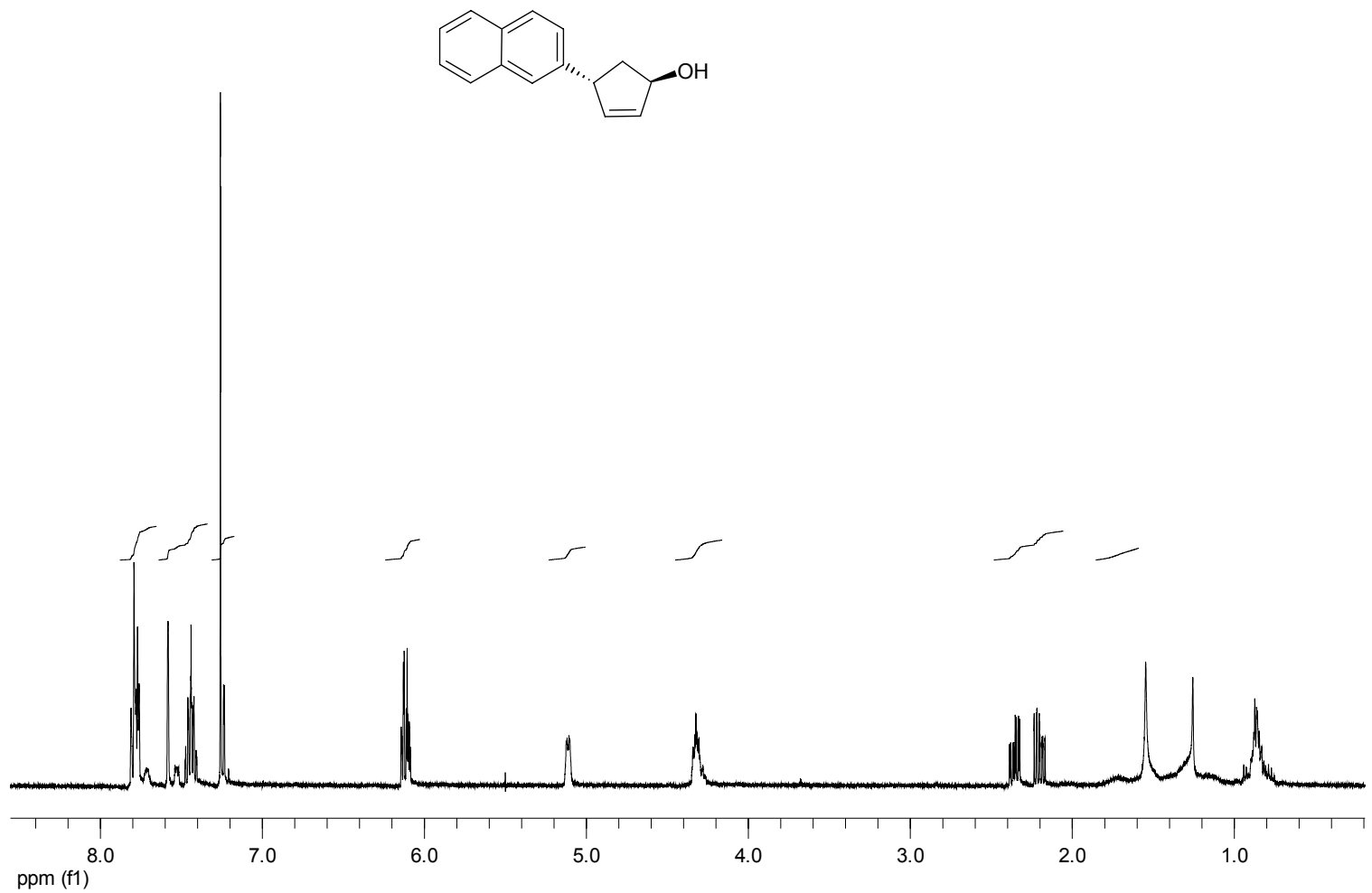

Rh-Catalyzed Allylic Substitution

S49

Menard, F.; Chapman, T.; Dockendorff, C. J.; Lautens, M. 
(5o) : (1R, 4R)-trans-4-(1-Naphthalenyl)-cyclopent-2-enol.

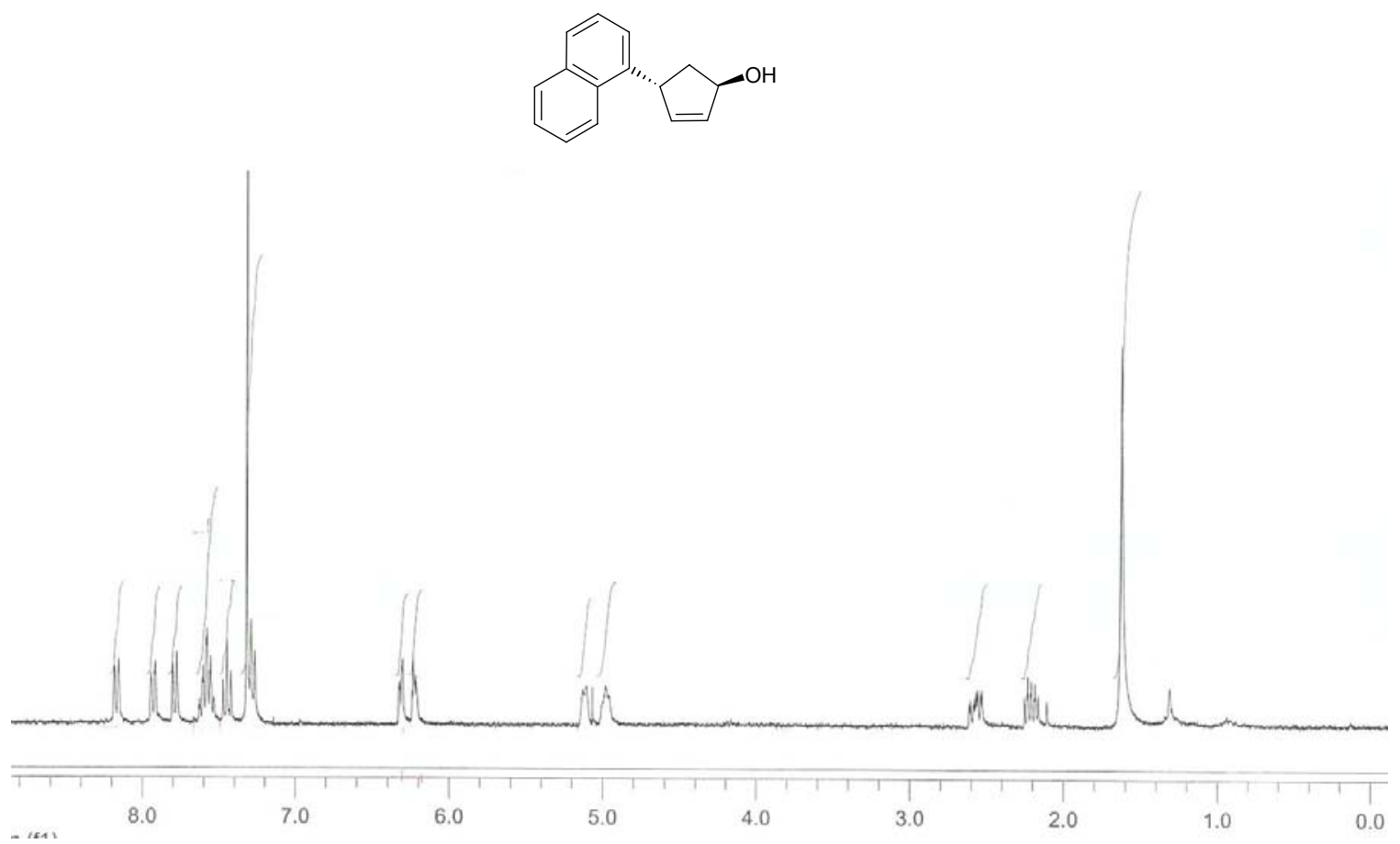

(5p) : (1R, 4R)-trans-4-(2-Methylphenyl)-cyclopent-2-enol.

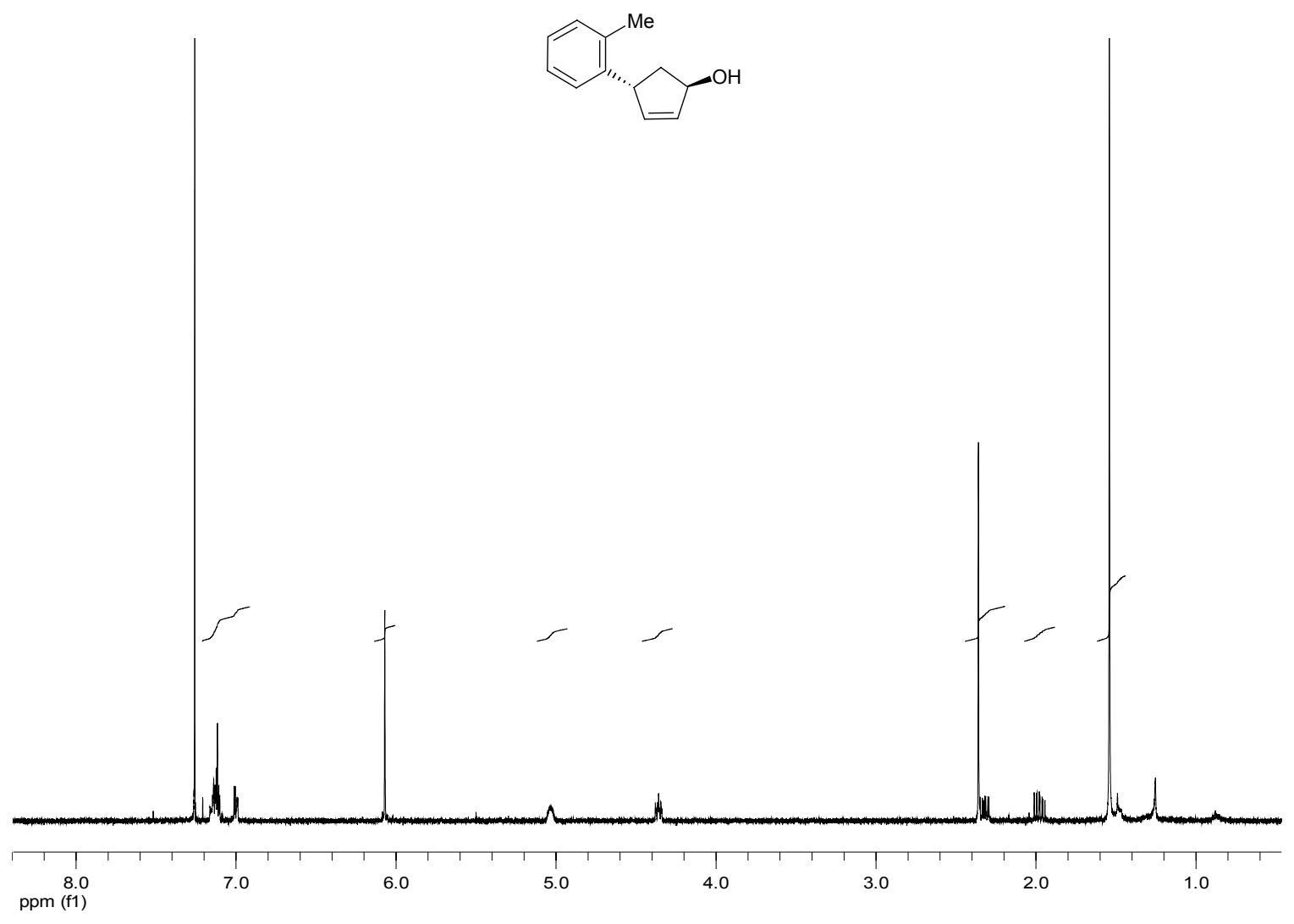




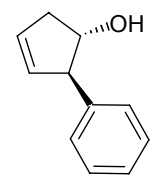

$(+)-(1 S, 2 R)-4 a$

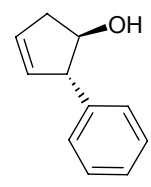

$(-)-(1 R, 2 S)-4 a$

HPLC Trace for racemic-4a:

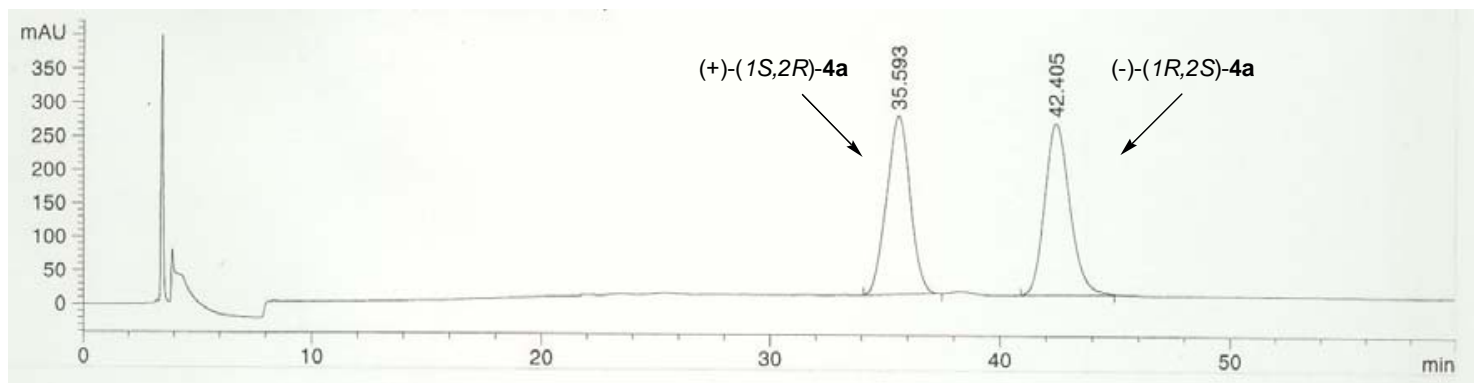

HPLC Trace for enantiomerically enriched-4a:

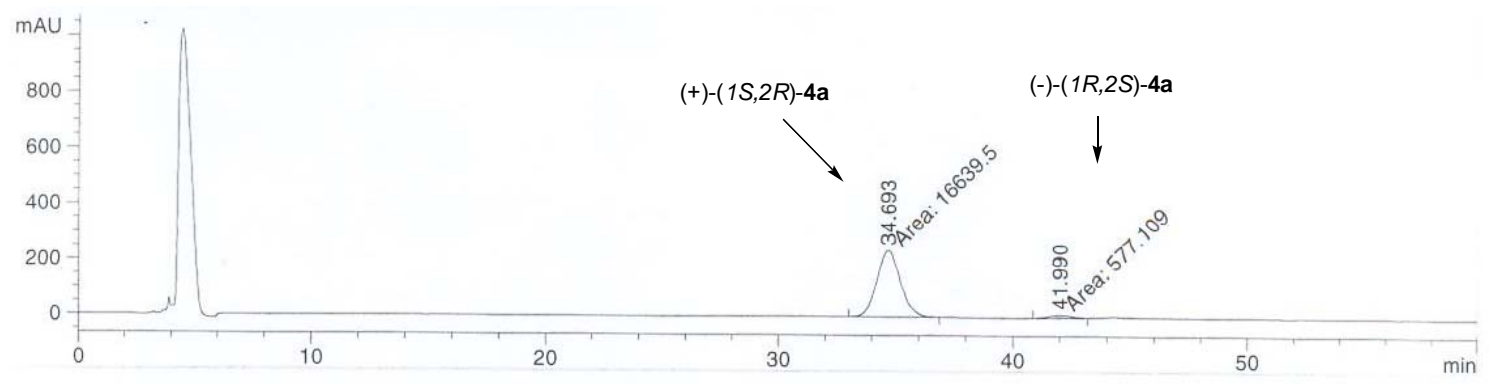

HPLC System Agilent 1100 Series

Column:Daicel Chiralcel OD-H

Eluent: $\quad$ 1:99, 2-Propanol:Hexane

Flow: $\quad 1.0 \mathrm{~mL} / \mathrm{min}$

Injection: $\quad 4.0 \mu \mathrm{L}$

Temp. col.: $\quad 30{ }^{\circ} \mathrm{C}$

Detector freq.: $\quad 210 \mathrm{~nm}$ 